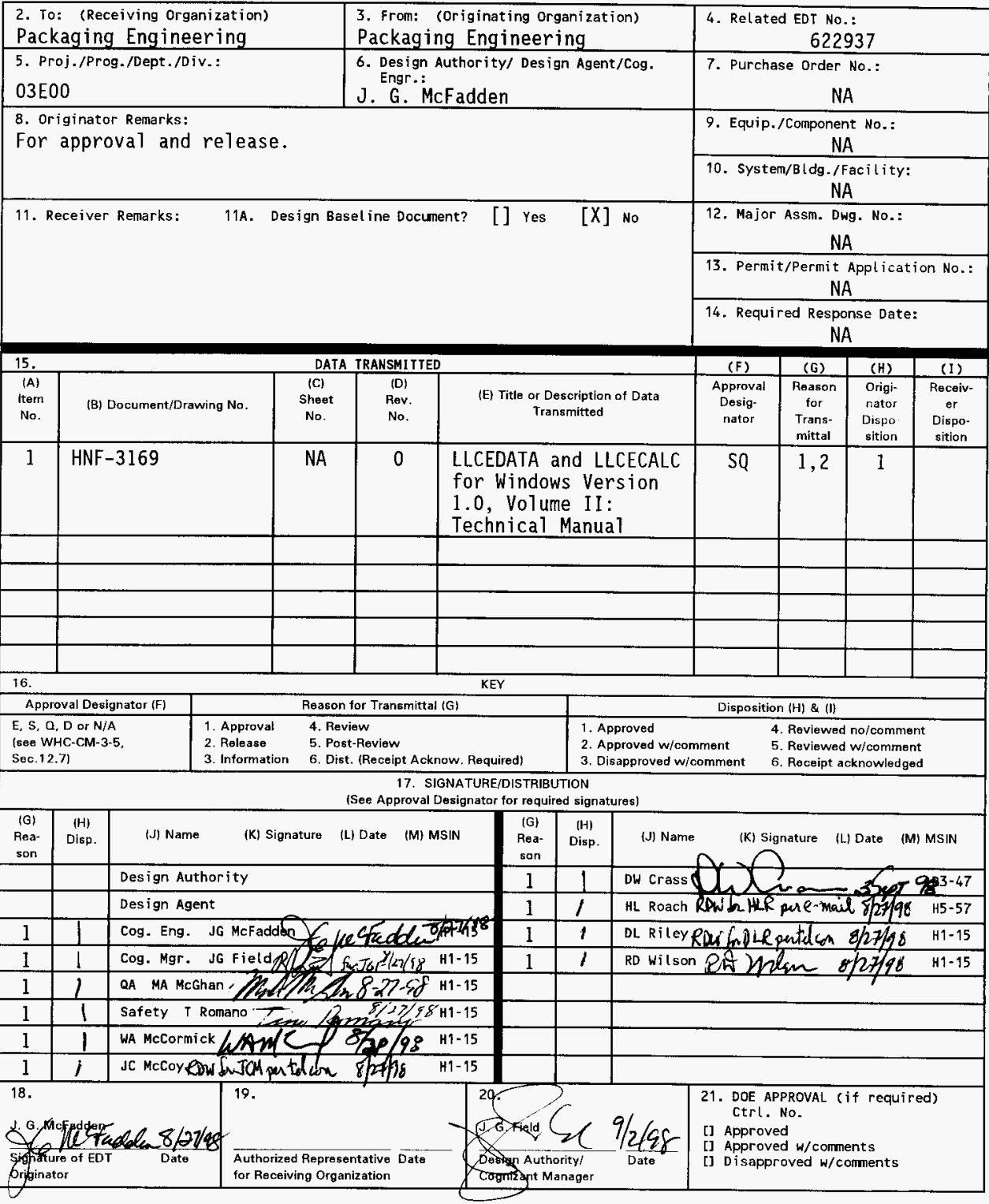

BD-7400-172-2 (05/96) GEF097 


\section{LLCEDATA and LLCECALC for Windows Version 1.0, Volume II: Technical Manual}

\section{J. G. McFadden}

Waste Management Federal Services, Inc., Northwest Operations

Richland, Washington 99352

for Fluor Daniel Hanford, Inc.

U.S. Department of Energy Contract DE-AC06-96RL13200

$\begin{array}{llll}\text { EDT/ECN: } & \text { EDT 622941 } & \text { UC: 513 UC-2000 } & \\ \text { Org Code: } & \text { 03E00 } & \text { Charge Code: D6309 } & \text { Task Order: GS080003 } \\ & & \text { Project: 772028 } & \text { Crosswalk: 40-007-001 } \\ \text { B\&R Code: } & \text { EW3130010 } & \text { Total Pages: 93 } & \end{array}$

Key Words: LLCE, Long-Length Contaminated Equipment, residual waste, characterization, GEA System, FRRDS

Abstract: LLCEDATA and LLCECALC for Windows Version 1.0 are userfriendly computer software programs that work together to determine the proper waste designation, handiing, and disposition requirements for Long-Length Contaminated Equipment. LLCEDATA and LLCECALC generate characterization information by integrating the acquisition of current tank waste data with the output from the Gamma Energy Analys is System, a component of the Flexible Receiver Radiation Detection System.

Documentation for LLCEDATA and LLCECALC for Windows is available in three volumes. Volume I is a user's manual, which is intended as a quick reference; Volume II is a technical manual; and Volume III is a software verification and validation document.

TRADEMARK DISCLAIMER. Reference herein to any specific commercial product, process, or service by trade name, tradenark, manufacturer, or otherwise, does not necessarily constitute or imply its endorsement, recommendation, or favoring by the United States Government or any agency thereof or its contractors or subcontractors.

Printed in the United States of America. To obtain copies of this document, contact: WHC/BCS Document Control Services, P.O. Box 1970, Mailstop H6-08, Richland WA 99352, Phone (509) 372-2420; Fax (509) 376-4989.
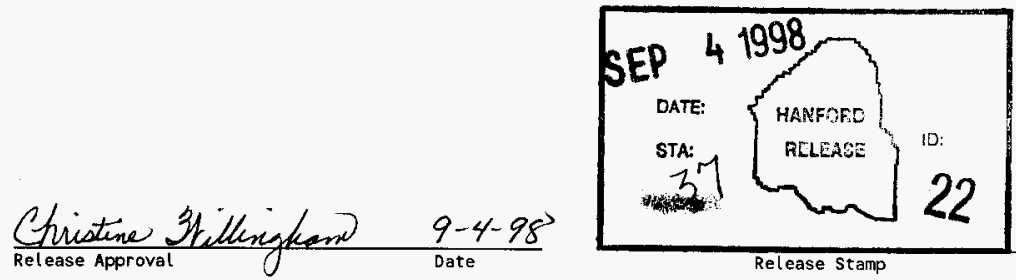

Approved for Public Release 


\section{LLCEDATA and LLCECALC FOR WINDOWS VERSION 1.0,}

VOLUME II: TECHNICAL MANUAL

Cognizant Engineer: J. G. McFadden

Prepared by: D. L. Riley, R. D. Wilson

Software: H. L. Roach

August 1998

Prepared for the U.S. Department of Energy

by

Engineering

Waste Management Federal Services, Inc., Northwest Operations 


\section{ACKNOWLEDGMENTS}

Verrsion 1.0 of LLCEDATA and LLCECALC for Windows was supported by the U.S. Department of Energy. LLCEDATA and LLCECALC for Windows' authors would like to thank the following people for their support on this project: A. D. Kirby, L. D. Kandt, and J. C. McCoy. 
This page intentionally left blank. 


\title{
LLCEDATA AND LLCECALC FOR WINDOWS VERSION 1.0, VOLUME II: TECHNICAL MANUAL
}

\author{
Cognizant Engineer: J. G. McFadden \\ Prepared by: D. L. Riley, R. D. Wilson \\ Software: H. L. Roach
}

\begin{abstract}
LLCEDATA and LLCECALC for Windows ${ }^{1}$ are user-friendly computer software programs that work together to determine the proper waste designation, handling, and disposition requirements for LongLength Contaminated Equipment (LLCE). LLCEDATA reads from a variety of databases to produce an equipment data file (EDF) that represents a "snapshot" of both the LLCE and the tank from which it originates. LLCECALC reads the EDF and the gamma assay (AV2) file that is produced by the Flexible Receiver Gamma Energy Analysis System. LLCECALC performs corrections to the AV2 file as it is being read and characterizes the LLCE. Both programs produce a variety of reports, including a characterization report and a status report. The status report documents each action taken by the user, LLCEDATA, and LLCECALC.
\end{abstract}

Documentation for LLCEDATA and LLCECALC for Windows is available in three volumes. Volume $\mathrm{l}$ is a user's manual, which is intended as a quick reference for both LLCEDATA and LLCECALC. Volume /I is a technical manual, which documents LLCEDATA and LLCECALC's methodology, structure, and format; provides sample calculations; discusses system limitations; and provides recommendations to the LLCE process. Volume III is a software verification and validation document.

LLCEDATA and LLCECALC for Windows are sponsored by the U.S. Department of Energy. They were developed and are maintained by Engineering, Waste Management Federal Services, Inc., Northwest Operations. Copies of the software and documentation are available by contacting Engineering, Waste Management Federal Services, Inc., Northwest Operations, Richland, Washington.

\footnotetext{
${ }^{4}$ Windows is a trademark of Microsoft Corporation.
} 
This page intentionally left blank. 


\section{FOREWORD}

This report presents the technical basis for LLCEDATA and LLCECALC for Windows. This report will fully document the methodology employed to characterize Long-Length Contaminated Equipment (LLCE) as it is retrieved from high-level waste tanks. The report will also discuss limitations and give recommendations for future improvements.

LLCEDATA and LLCECALC are software programs developed and maintained by Engineering, Waste Management Federal Services, Inc., Northwest Operations, Richland, Washington, for the U.S. Department of Energy. Documentation for the software programs exists in the form of LLCEDATA and LLCECALC for Windows Version 1.0, Volume I: User's Manual; LLCEDATA and LLCECALC for Windows Version 1.0, Volume II: Technical Manual; and LLCEDATA and LLCECALC for Windows Version 1.0, Volume III: Software Verification and Validation. The reader is urged to have all three documents available for reference when becoming familiar with the software prior to use. The software is available from Engineering by calling 509-376-0610 or 509-376-7111. Corrections and suggestions for additions or modifications to LLCEDATA and LLCECALC for Windows are welcome. 
This page intentionally left blank. 


\section{CONTENTS}

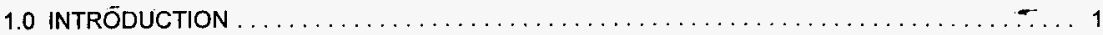

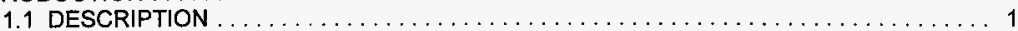

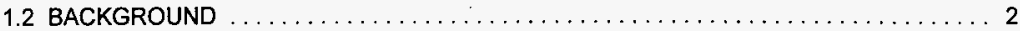

1.3 LLCEDATA AND LLCECALC PROGRAM SCOPE $\ldots \ldots \ldots \ldots \ldots \ldots \ldots \ldots \ldots \ldots \ldots 2$

1.4 LIMITATIONS OF LLCEDATA AND LLCECALC FOR WINDOWS $\ldots \ldots \ldots \ldots \ldots \ldots \ldots$.

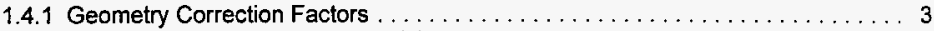

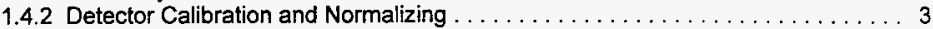

1.4.3 Single Gamma Emitter Assayed and Ratios Inferred . . . . . . . . . . . . . . 4

1.4.4 Single-Layer Model, LLCEDATA Tank Database $\ldots \ldots \ldots \ldots \ldots \ldots \ldots \ldots 4$

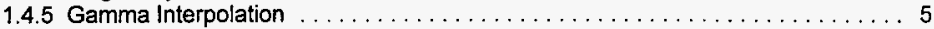

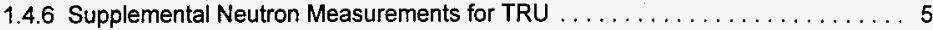

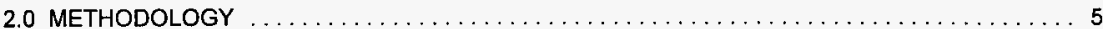

2.1 GAMMA SPECTRAL ANALYSIS SYSTEM $\ldots \ldots \ldots \ldots \ldots \ldots \ldots \ldots \ldots \ldots \ldots \ldots \ldots \ldots$

2.2 DETERMINATION OF LLCE GAMMA ACTIVITY $\ldots \ldots \ldots \ldots \ldots \ldots \ldots \ldots \ldots \ldots \ldots$

2.3 GAMMA ASSAY FILE DATA ANALYSIS PROCESS $\ldots \ldots \ldots \ldots \ldots \ldots \ldots \ldots \ldots$

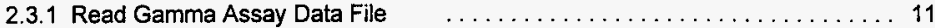

2.3.2 Check and Correct Positions in Gamma Assay Records . . . . . . . . . . . 12

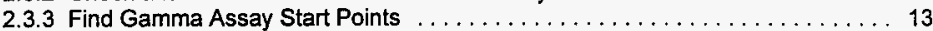

2.3.4 Check and Correct for Superseded Records . . . . . . . . . . . . . . . . 15

2.3.5 Applying Geometry Correction Factors For ${ }^{137} \mathrm{Cs} \ldots \ldots \ldots \ldots \ldots \ldots \ldots \ldots$

2.3.6 Applying Detector Shielding Correction Factors for ${ }^{137} \mathrm{Cs} \ldots \ldots \ldots \ldots \ldots \ldots$

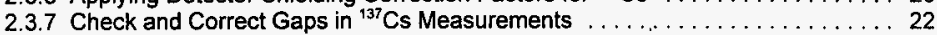

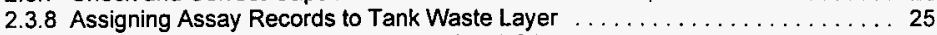

2.3.9 Calculating Measured Curie Content for LLCE $\ldots \ldots \ldots \ldots \ldots \ldots \ldots \ldots \ldots$

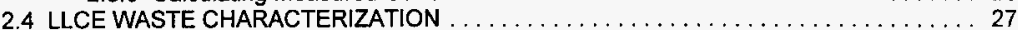

2.4.1 Calculate Radionuclide and Chemical Content . . . . . . . . . . . . . 29

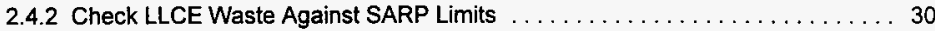

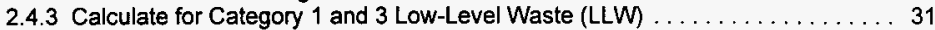

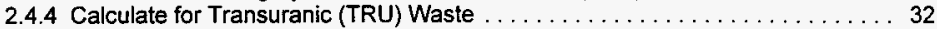

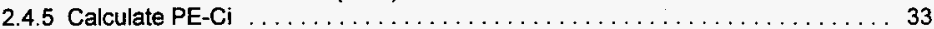

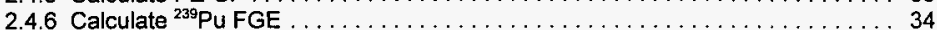

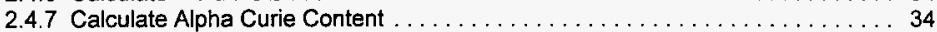

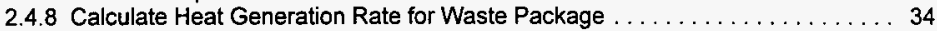

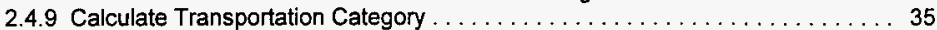

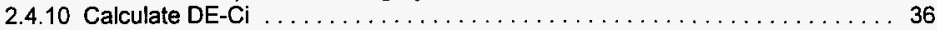

2.4.11 Calculate for Hazardous Waste Codes . . . . . . . . . . . . . . . . . 36

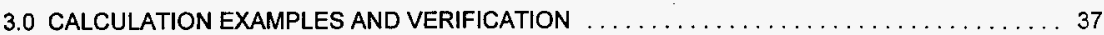

3.1 SELECTED CALCULATION EXAMPLES FROM THE ANALYZE GAMMA ASSAY

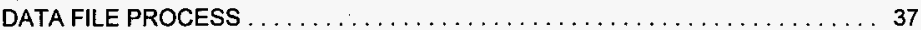

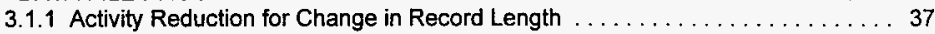

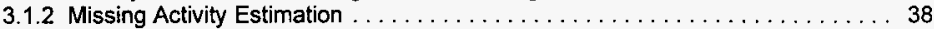

3.1.3 Weighted Averaged Activity From Three Detectors . . . . . . . . . . . . 39

3.1.4 Calculation of the Shielding Correction Factors for Detectors 2 and $3 \ldots \ldots .40$

3.2 CALCULATION EXAMPLES FROM THE LLCE WASTE CHARACTERIZATION

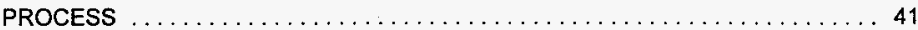

3.2.1 Calculate Radionuclide and Chemical Content $\ldots \ldots \ldots \ldots \ldots \ldots \ldots \ldots 42$

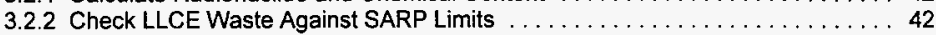

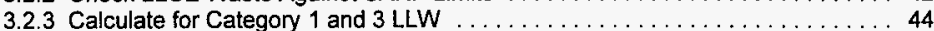

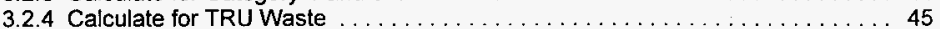

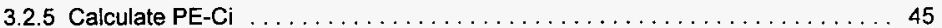

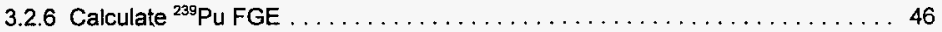

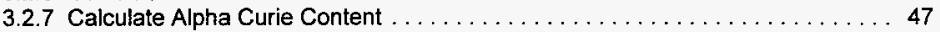




\section{CONTENTS (cont.)}

3.2.8 Calculate Heat Generation Rate for Waste Package . . . . . . . . . . . . . . . . 47

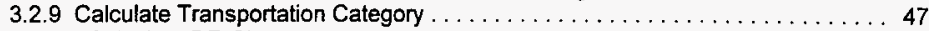

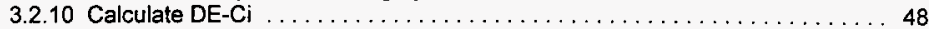

3.2.11 Calculate for Hazardous Waste Codes . . . . . . . . . . . . . . . 48

3.2.12 Summary of LICE Waste Characterization Calculations . . . . . . . . . . 49

4.0 EDF AND MAIN DATABASE FILE FORMAT $\ldots \ldots \ldots \ldots \ldots \ldots \ldots \ldots \ldots \ldots \ldots \ldots \ldots \ldots$

4.1 ORIGINATING DATABASE FILE: LLCEINFO.BIN $\ldots \ldots \ldots \ldots \ldots \ldots \ldots \ldots \ldots \ldots .49$

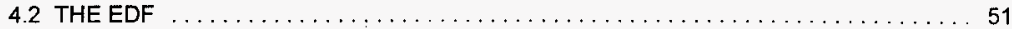

5.0 SUMMARY AND RECOMMENDATIONS $\ldots \ldots \ldots \ldots \ldots \ldots \ldots \ldots \ldots \ldots \ldots \ldots \ldots \ldots$

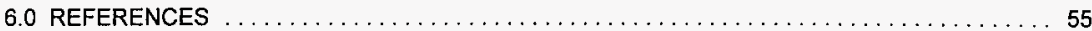

APPENDICES

A1 LLCEDATA AND LLCECALC DATABASE AND LLCEINFO.BIN DESCRIPTION $\ldots \ldots \ldots \ldots$ A-1

A2 LLCEDATA AND LLCECALC DATABASE FILE STRUCTURE $\ldots \ldots \ldots \ldots \ldots \ldots \ldots \ldots \ldots$ A-2

A3 LLCEDATA AND LLCECALC CONFIGURATION FILES $\ldots \ldots \ldots \ldots \ldots \ldots \ldots \ldots \ldots \ldots$ A-5

A4 LLCEDATA AND LlCECALC DATABASE TABLE STRUCTURES $\ldots \ldots \ldots \ldots \ldots \ldots \ldots \ldots$ A $\ldots \ldots$

B DESCRIPTION OF THE AV2 FILE FORMAT $\ldots \ldots \ldots \ldots \ldots \ldots \ldots \ldots \ldots \ldots \ldots \ldots \ldots \ldots$

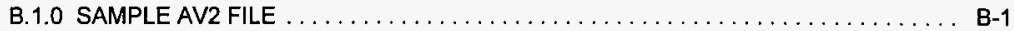

B.2.0 STATISTICALLY WEIGHTED AVERAGE FOR AV2 ACTIVITY MEASUREMENTS ... B-2

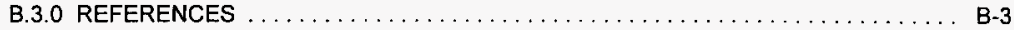

\section{LIST OF TABLES}

3-1 Appropriate Portions of EDF Database Tables for Sample Calculations $\ldots \ldots \ldots \ldots \ldots \ldots 43$

4-1 Description of /lceinfo.bin (//ceinfo mab) Database Tables $\ldots \ldots \ldots \ldots \ldots \ldots \ldots \ldots \ldots$

4-2 Equipment Data File (EDF) Database Tables $\ldots \ldots \ldots \ldots \ldots \ldots \ldots \ldots \ldots \ldots \ldots \ldots \ldots \ldots \ldots$ 


\section{LIST OF TERMS}

$\begin{array}{ll}\text { AV2 } & \text { gamma assay (file) } \\ \text { CAS } & \text { Chemical Abstracts Service } \\ \text { CD-ROM } & \text { compact disk read-only memory } \\ \text { DE-Ci } & \text { dose equivalent curie } \\ \text { EDF } & \text { equipment data file } \\ \text { FGE } & \text { fissile gram equivalent } \\ \text { FRRDS } & \text { Flexible Receiver Radiation Detection System } \\ \text { GEA } & \text { Gamma Energy Analysis (System) } \\ \text { ID } & \text { inside diameter } \\ \text { ISB } & \text { interim safety basis } \\ \text { LLCE } & \text { Long-Length Contaminated Equipment } \\ \text { LLW } & \text { low-level waste } \\ \text { MB } & \text { megabyte } \\ \text { NRC } & \text { U.S. Nuclear Regulatory Commission } \\ \text { OD } & \text { outside diameter } \\ \text { PE-Ci } & \text { plutonium equivalent curie } \\ \text { SARP } & \text { safety analysis report for packaging } \\ \text { TRU } & \text { transuranic }\end{array}$


This page intentionally left blank. 


\section{LLCEDATA AND LLCECALC FOR WINDOWS VERSION 1.0, VOLUME II: TECHNICAL MANUAL}

\subsection{INTRODUCTION}

\subsection{DESCRIPTION}

The Long-Length Contaminated Equipment (LLCE) disposal effort involves retrieval, handling, and disposal of the LLCE from any one of the 177 underground waste storage tanks, catch tanks, and lift stations. When the LLCE is retrieved from the tanks, every effort is made to remove any residual tank waste that is on the equipment surfaces. It is expected that some amount of tank waste will remain on the LLCE after retrieval. The radionuclide and chemical content of this residue must be assessed to determine the proper waste designation, handling, and disposition requirements for the LLCE.

LLCEDATA and LLCECALC have been written to characterize the contamination that remains on an LLCE after it has been washed by water jets during retrieval from the high-level waste tanks at Hanford. Their purpose is to generate characterization information by integrating the acquisition of current tank waste data with the output from the Gamma Energy Analysis (GEA) System, a component of the Flexible Receiver Radiation Detection System (FRRDS). LLCEDATA and LLCECALC allow for the quick, efficient, reproducible, and well documented characterization of an LLCE.

In order for the LLCECALC program to perform an LLCE characterization, LLCEDATA must first be used to create an equipment data file (EDF). The LLCEDATA program reads from a variety of databases, discussed in Section 4.0, to assemble the EDF. The LLCEDATA-produced EDF is a "snapshot" of the LLCE and its originating tank. The completed EDF, ready to be used by LLCECALC, contains complete information concerning the LLCE.

In addition to the LLCEDATA-generated EDF, the LLCECALC program requires a gamma assay file, known as the AV2 file. The AV2 file is produced by the gamma isotope measurements from the GEA System. Once the EDF and AV2 file have been defined in LLCECALC, the LLCE characterization is ready to begin.

LLCEDATA and LLCECALC are Microsoft ${ }^{1}$ Windows $^{2}$ Visual Basic ${ }^{3}$ executable programs that are menu driven and are easy for the user to navigate.

This document presents the technical basis and calculational methodology of the characterization and describes the role of each program in this process. The limitations of the contamination characterization are presented. The limitations are not associated with the software, but rather are due to assumptions in the methodology and constraints imposed by the measurement process.

The software has been extensively tested, and by independent confirmation of the algorithms, LLCECALC has been shown to faithfully apply the characterization methodology. The testing and validation process is presented in the companion document, LLCEDATA and LLCECALC for Windows Version 1.0, Volume III: Verification and Validation.

Another companion, Volume I: User's Manual, is intended for quick reference. It is to be used as an aid for learning to use LLCEDATA and LLCECALC for Windows and as a guide to the programs' uses.

\footnotetext{
${ }^{1}$ Microsoft is a trademark of Microsoft Corporation.

${ }^{2}$ Windows is a trademark of Microsoft Corporation.

${ }^{3}$ Visual Basic is a trademark of Microsoft Corporation.
} 
The user's manual contains a discussion of all the options available to the user for each menu found in both LLCEDATA and LLCECALC.

\subsection{BACKGROUND}

The software programs LLCEDATA and LLCECALC have been under development for about 2 years (Roach 1995). LLCEDATA is meant to provide a comprehensive yet simple methodology to identify and select LLCE candidates, identifying both their specific characteristics and the tank constituents they are pulled from. Databases are maintained in LLCEDATA to identify each LLCE; each tank's constituent content; and each container approved by HNF-SD-TP-SARP-013, Safety Analysis Report for Packaging (Onsite) Long-Length Contaminated Equipment Transport System (McCormick 1997). The LLCECALC program is meant to provide for near "real-time" gamma activity analysis and contamination characterization of an LLCE as it is retrieved from the underground high-level waste storage tanks at the 200 Areas of the Hanford Site.

Conceptual design for the gamma measurement system was completed by early 1991 (Troyer 1998), and an initial version of the hardware was constructed and installed in the retrieval equipment for testing by summer 1995 (Troyer 1998). In 1995 and 1996 the measurement system acquired gamma assay data for six LLCE thermocouple trees as they were retrieved from waste tanks $A Z-101$ and $A W-104$. The resulting gamma data were processed manually using a spreadsheet approach for total LLCE contamination and for the characterization of the waste for transportation and disposal. The characterization results have been summarized (Gedeon 1996), and specific manual examples of radioisotope inventories are available (Kessler 1996).

LLCEDATA and LLCECALC have been developed to replace this manual analysis approach with an automated approach based on the same methodology. However, each such computer analysis will be well documented; assumptions made concerning the treatment of anomalies in the gamma data will be clearly defined; and results will be quickly available to those retrieving the LLCE, allowing for timely decisions concerning packaging, transportation, and disposal.

\subsection{LLCEDATA AND LLCECALC PROGRAM SCOPE}

It is the purpose of LLCEDATA and LLCECALC for Windows Version 1.0 to generate characterization information by integrating the acquisition of current tank waste data, from LLCEDATA, with the output from the GEA System, a component of the FRRDS, as used by LLCECALC. LLCEDATA and LLCECALC provide an efficient mechanism to characterize an LLCE.

LLCEDATA maintains several databases for its use. The three main databases are (1) a database of all known LLCE, (2) a database of tank chemical and radionuclide constituents, and (3) a database of containers approved by McCormick (1997). It is important that the databases in LLCEDATA be kept up to date. The LLCEDATA code custodian has the responsibility to maintain the databases. The user of LLCEDATA should check with the code custodian to verify that the user has the latest version of the code. This limitation may be temporarily avoided by using the ability of LLCEDATA to edit any and all fields used in the specification of an LLCE, including a tank's chemical or radionuclide contents. The user should note that these changes or additions that are made are specific to the EDF being edited and are not changed or stored in the actual main databases. The code custodian is required to make the permanent changes to the databases.

In order for the LLCECALC program to perform an LLCE characterization, LLCEDATA must first be used to create an EDF. The LLCEDATA-produced EDF is a "snapshot" of the LLCE and its originating tank. The completed EDF, ready to be used by LLCECALC, contains complete information concerning the LLCE. In addition to the LLCEDATA-generated EDF, the LLCECALC program requires the AV2 gamma file. Once the EDF and AV2 file have been defined in LLCECALC, the LLCE characterization is ready to begin. 
LLCECALC performs two main functions: process gamma and characterization. The first function, process gamma, is performed as LLCECALC reads the supplied AV2 file that was created by the FRRDS. During this process, nine steps are performed while LLCECALC reads, adjusts, and calculates the total ${ }^{137} \mathrm{Cs}$ content of the LLCE. User intervention is typically required during these steps, with default values supplied in each case. The second function, characterization, performs 11 steps while LLCECALC calculates the actual characterization of the LLCE.

LLCEDATA and LLCECALC allow several reports to be previewed or printed. LLCEDATA allows the user to print the following reports: LLCE Physical Data, Tank Characterization Database, and LLCE Geometry Factors. LLCECALC allows the user to print the LLCE Characterization Report. In addition to these reports, both programs maintain a current running status of all activity performed. This status report can be viewed or printed at any time. The status report is a running record of all activities performed by both the user and the two programs. This report can serve as a historical record to show exactly who, what, when, where, and how an LLCE was analyzed. The final characterization report, produced by LLCECALC, can be used for making onsite decisions concerning disposition of the retrieved equipment.

\subsection{LIMITATIONS OF LLCEDATA AND LLCECALC FOR WINDOWS}

There are certain limitations imposed by the process used by LLCEDATA and LLCECALC. Some of these limitations can be eliminated by improvements that are proposed in Section 5.0, "Summary and Recommendations." The software programs themselves, LLCEDATA and LLCECALC, cause none of these limitations. The limitations are in the process of the actual gamma measurements and the subsequent analysis methods. The resulting LLCE contamination characterizations are affected by these limitations. It is difficult to assess additional uncertainty in the characterizations that are surely contributed by the limitations.

The limitations are fully discussed below and can be summarized as follows:

- Calibration limitations: Geometry Correction Factors

- $\quad$ Only detector No. 1 calibrated; others normalized to detector No. 1 response

- Only ${ }^{137} \mathrm{Cs}$ currently assayed; all other LLCE characterization ratio inferred

- $\quad$ Single-Layer Tank Model in LLCEDATA's current database

- Gamma Interpolation

- No neutron measurement for transuranic (TRU) identification.

\subsubsection{Geometry Correction Factors}

The gamma assay system is presently calibrated for gamma activity using a point source of known activity. An actual LLCE presents an extended gamma source to the detector system and is detected less efficiently. The so-called "geometry correction factors" are used to account for this difference in efficiency. Geometry correction factors are determined by computer simulation and are appropriate only for LLCE with negligible self-absorption for the source gammas and for the shielding and collimation used in the computer models. Geometry correction factors that do not account for selfabsorption will not, for example, be appropriate for other types of equipment, such as pumps (Nelson 1998). For these types of LLCE, new geometry correction factors will be required that will take this into account (Nelson 1998). The LLCEDATA program allows the user to input new geometry correction factors into the equipment database. Presently, the database only contains geometry correction factors for thermocouple trees, which are assumed to be free of self-absorption effects.

\subsubsection{Detector Calibration and Normalizing}

The calibration procedure presently in use with the gamma assay system is unable to perform an adequate efficiency calibration of the two shielded detectors. Therefore, only the unshielded detector is 
efficiency calibrated for the gamma ray from the decay of ${ }^{137} \mathrm{Cs}$ (the gamma ray is actually emitted by the short-lived progeny ${ }^{137} \mathrm{Ba}$ metastable). The two shielded detector responses must be compared to the unshielded detector response using the actual LLCE gamma data. This comparison efficiency is determined in the LLCECALC program. Experience has shown that these comparison efficiency factors are variable from one LLCE retrieval to the next, especially for detector 3 , and the process is judged to contribute some uncertainty in the overall activity calculation.

The calibration procedure should be upgraded in future versions of the FRRDS to eliminate this limitation so that all three detectors can represent independent measurements of LLCE gamma activity. A consequence of this limitation is that one of the measurement objectives of the system is lost--the ability to measure azimutha! variations in gamma activity around the circumference of the LLCE. A recommended calibration procedure that eliminates the need for both the geometry correction factors. and for the efficiency factors is presented in Section 5.0.

\subsubsection{Single Gamma Emitter Assayed and Ratios Inferred}

The gamma assay system presently processes only the gamma signature from ${ }^{137} \mathrm{Cs}\left({ }^{137 m} \mathrm{Ba}\right)$. There are also detected signatures from ${ }^{60} \mathrm{Co}$ and ${ }^{154} \mathrm{Eu}$, but these are not used in characterizing the LLCE activity from other radionuclides. Rather, the tank waste database is used to derive a ratio of all other radionuclide activities to that measured for ${ }^{137} \mathrm{Cs}$. In using these ratios, it is assumed that the contamination remaining on the LLCE has the same mix of radionuclides as the tank waste layer in which it was located before retrieval. If there are several layers of tank waste and radionuclide ratios are available in the database for each layer, then the LLCE contamination at each axial position on the LLCE equipment for a given radionuclide is determined relative to ${ }^{137} \mathrm{Cs}$ using the data for the appropriate adjacent layer.

The user is cautioned that there has been no experimental verification that the ratio of each radionuclide activity to the measured ${ }^{137} \mathrm{Cs}$ activity is the same as that determined from the tank waste database. In fact, there is evidence to the contrary for the case of ${ }^{60} \mathrm{Co}$. For ${ }^{60} \mathrm{Co}$ there is at least one instance, from actual LLCE retrieval data, where the ratio of ${ }^{60} \mathrm{Co}$ to ${ }^{137} \mathrm{Cs}$ was measured to be variable along the length of the LLCE (Troyer et al. 1998).

There should be samples taken of actual residual LLCE contamination, and the radionuclide ratios relative to ${ }^{137} \mathrm{Cs}$ should be measured for those contaminants critical to the characterization process. These measured ratios, when compared to those for the tank waste, will provide a measure of the uncertainty caused by this limitation in the methodology.

The ratio uncertainty may be the most serious limitation of all those identified.

\subsubsection{Single-Layer Model, LLCEDATA Tank Database}

Although there are typically two or more layers of waste in the tanks, the tank waste characterization database employed by LLCEDATA contains only a single waste layer for each tank containing radionuclide activities appropriate for a homogenized single layer of average composition. This is intended only as a starting point for the tank waste characterization data. It is anticipated that in most cases the tank waste data in the database will be replaced by the most current and accurate tank waste data available prior to when an LLCE is retrieved from a waste tank. The updated tank waste data will be entered into the database using the LLCEDATA program. LLCE is designed to allow for the definition of multiple tank waste layers. With a multi-layer tank waste database, a more realistic analysis of the LLCE contamination can be done with LLCECALC by assigning radionuclide activity relative to the

${ }^{137} \mathrm{Cs}$ measurement for each position according to the adjacent layer of waste. The second verification case in Volume I: User's Manual presents an example of a five-layer synthetic waste tank. This synthetic case is shown to be properly characterized in Volume III: Software Verification and Validation. 


\subsubsection{Gamma Interpolation}

There are instances where gamma data are missing for what are usually small intervals along the length of the LLCE. The program LLCECALC is designed to place an interpolated value of ${ }^{137} \mathrm{Cs}$ activity in this interval unless the user provides a value when prompted. This is considered a minor, and probably unavoidable, limitation to the system. Only for instances where the ${ }^{137} \mathrm{Cs}$ activity is strongly varying with length when the gap in data is encountered will this interpolation method prove inadequate.

\subsubsection{Supplemental Neutron Measurements for TRU}

The TRU radionuclide contamination of the $L L C E$ is presently inferred from the measured ${ }^{137} \mathrm{Cs}$ activity, assuming that it occurs in the same ratio to ${ }^{137} \mathrm{Cs}$ on the LLCE as in the tank waste. As discussed in limitation 1.4.3, there have been no measurements to validate the ratio assumption. However, the presence of TRU can be detected with neutron measurements. The decision as to whether the LLCE is suspect TRU waste has a significant impact on the waste disposal process. The absence of neutron LLCE measurements is considered a serious limitation because it introduces a large degree of uncertainty in the present method of characterizing the LLCE as suspect TRU.

\subsection{METHODOLOGY}

LLCECALC performs two main functions, process gamma and characterization. The process gamma phase starts by reading the EDF and AV2 file and attempts to determine the ${ }^{137} \mathrm{Cs}$ activity along the length of the LLCE. After application of the methodology outlined in Section $2.3, a{ }^{137} \mathrm{Cs}$ activity will have been assigned to each 1 -ft interval of the LLCE. These activities are simply summed over the length of the LLCE to obtain the total ${ }^{137} \mathrm{Cs}$ activity.

The second function of LLCECALC, characterization, starts with the total ${ }^{137} \mathrm{Cs}$ activity and determines the activity for all other radionuclides that contaminate the LLCE. The contaminant activities are determined by using the ratios of these radionuclide activities to the measured ${ }^{137} \mathrm{Cs}$ activity for the layer of tank waste adjacent to each position on the LLCE. LLCECALC assigns a waste layer to each LLCE activity record. The characterization process consists of comparing the various computed radionuclide activities to limits that affect the encapsulation, transportation, and disposal of the LLCE. The algorithms that implement the actual waste characterization methodology are discussed in Section 2.4 .

The reader should refer to the companion manuals, LLCEDATA and LLCECALC for Windows Version 1.0, Volume l: User's Manual, for instructions on carrying out this analysis with LLCEDATA and LLCECALC, and Volume III: Software Verification and Validation. Volume III demonstrates how the process gamma and characterization algorithms and methodology, discussed in Sections 2.3 and 2.4, are verified by independent spreadsheets that confirm the results obtained from LLCECALC are identical to the results from the independent calculations. The detailed discussions presented in Volume III for both software programs demonstrate independent confirmation of the algorithms used by LLCECALC.

The following sections will present the technical basis and calculational methodology of the process gamma and characterization phases of LLCECALC.

\subsection{GAMMA SPECTRAL ANALYSIS SYSTEM}

The intent of the gamma analysis system is to measure activities for all radionuclides producing detected gamma rays and to provide these activities over a wide dynamic range and at approximately one-foot intervals along the entire length of LLCE. This is done as the LLCE is being retrieved from the waste tank, at about $1 \mathrm{ft} / \mathrm{min}$. Detectors arrayed around a horizontal platform at equally spaced azimuthal 
intervals were to provide independent activity measurements so that any variation in contamination with azimuthal position would be detected and properly averaged for each axial position along the length of the LICE. The detectors were to provide high-energy resolution so that the multitude of gamma-ray peaks could be separated from each other and properly assigned to parent radionuclides. For this reasontlighPurity Germanium (HPGe) detectors with identical unshieided efficiencies were procured for the system.

The implemented gamma measurement system is described in a recent paper (Troyer et al. 1998). A set of three HPGe detectors is arranged in a horizontal plane, separated azimuthally by $120^{\circ}$. Each HPGe detector is surrounded by a lead shield to prevent the detection of gamma rays from directions other than the approximate $1-\mathrm{ft}$ axial interval bisected by the horizontal plane of the LLCE at that location. The detectors are identical, and the original intent was to have identical collimators so they would have nearly identical efficiencies and could all be calibrated with a single point source to give activity in curies from the measured gamma photopeak intensity. However, the detectors did not exhibit sufficient dynamic range to accommodate all activities encountered with actual contaminated LLCE. Therefore, lead shielding was placed in the collimator regions of two of the detectors to reduce their efficiency.

The present HPGe detector configuration is that detector No. 1 has an unshielded collimator region; detector No. 2 has an intermediate efficiency due to $4.8 \mathrm{~cm}$ of lead in the collimator region; and detector No. 3, with the lowest efficiency, has $7.6 \mathrm{~cm}$ of lead in the collimator region. The wide dynamic range is now achieved, but detectors 2 and 3 can no longer be calibrated with the point source because of their low efficiency. This deficiency in the calibration process has for the present been overcome by adding to the program LLCECALC the capability of computing efficiency factors for detectors 2 and 3 , relative to detector 1 , by use of the actual measured LLCE gamma data. These factors are called "shielding correction" factors in the LLCECALC program. The result is a loss of independence in the measurements by detectors No. 2 and No. 3. A method is proposed in Section 5.0 to recover this measurement independence by using a new calibration procedure.

Detector No. 1 is calibrated for its gamma efficiency by using a point source with known activity. This source is placed in the horizontal plane of the detection system at the location occupied by the LLCE when being retrieved. The nominal efficiency of each detector is $15 \%$ relative to a 3 -in.-diameter by 3 -in.long sodium iodide detector for a point source along its axis with an energy of $1332 \mathrm{keV}$. The gamma spectra acquired by the detectors are processed for gamma-ray activity by software procured from Edgerton, Germeshausen \& Grier (EG\&G) Idaho (Killian and Hartwell 1988, Killian and Femec 1992, Killian and Sorensen 1992).

Since the gamma activity on the LLCE is in the approximate form of a line source, a correction must be made to the detector efficiency obtained by the point source. This point-source-to-fine-source correction factor, called the "geometry correction" factor in LLCECALC, is determined from computer modeling as described by Troyer et al. (1998). The geometry correction factor is greater than unity and is largest for the least efficient detector, detector No. 3 .

There is presently a single set of geometry correction factors available to the user-detector No. 1: 1.481 ; detector No. 2: 1.934 ; and detector No. 3: 1.957 . These correction factors are suitable only for an LLCE for which it is valid to assume that negligible self-absorption of the ${ }^{137} \mathrm{Cs}\left({ }^{137 m} \mathrm{Ba}\right)$ gamma ray takes place in the equipment itself. When this is the case, the same values for the geometry correction factors can be used along the entire length of the LLCE. The values presently in use are appropriate only for the current shielding configuration. If the lead in the collimated region of the detectors is changed, new geometry factors must be computed. Also, if a pump or other equipment with significant self-absorption effects is to be retrieved, then new values of the geometry factors must be computed.

Such equipment may also require added information or assumptions concerning source location within the equipment.

The gamma measurement system spectrum analysis software identifies gamma-ray peaks, determines their intensities (counts/s) and \% error, and associates the spectral record with the nearest crane position reported to the computer that controls data acquisition (Troyer et al. 1998). This software also computes a gamma activity for each identified radionuclide based on the supplied efficiency 
calibration data and a radionuclide decay gamma library. Presently, as discussed above, only detector 1 contains a valid efficiency factor and hence only for detector 1 has a point source efficiency factor been correctly applied to the ${ }^{137} \mathrm{Cs}\left({ }^{137 \mathrm{~m}} \mathrm{Ba}\right)$ peak intensity data.

Although this measurement analysis software reports intensities for all valid gamma peaks, only the ${ }^{137} \mathrm{Cs}\left({ }^{137 \mathrm{~m}} \mathrm{Ba}\right)$ intensity at $662 \mathrm{keV}$ is used by the present methodology. A gamma assay data file (AV2) is created by the measurement system that consists of a set of activity records for the three detectors for all measurement positions of the crane that is retrieving the LLCE. Details of spectrum analysis and data record creation are documented in WHC-SD-W151-UM-001, Flexible Receiver Radiation Detection System (FRRDS) Users Manual (WHC 1996). These activity records are later related to location on the LLCE by the program LLCECALC. These data files are ASCII files with an extension of AV2. As of this writing, there are only six such files in existence from LLCE retrievals completed during 1995 and 1996. These files are kept separately from the LLCE and waste tank database information, which is kept in a file identified with the extension EDF. The program LLCECALC requires an EDF for the particular LLCE and waste tank and an AV2 file of the gamma assay data.

\subsection{DETERMINATION OF LLCE GAMMA ACTIVITY}

After the LLCE has been retrieved and washed and gamma measurements have been completed, it is possible to perform the gamma activity and contamination characterization analysis. At completion of the gamma measurements, an AV2 file of gamma activity (or apparent activity in the case of detectors No. 2 and No. 3) for each 60-second measurement along the length of the LLCE is available. The crane pulls the LLCE at approximately $1 \mathrm{ft} / \mathrm{min}$ so that each gamma measurement samples about $1 \mathrm{ft}$ of the LLCE's length. As the crane retrieves the LLCE, a position signal is sent to the controlling computer at about 4-in. intervals. The AV2 file associates each set of gamma data with the nearest crane position signal following completion of the gamma count. In this way, a crane position is assigned to each gamma data record. See Appendix B for discussion and an example of an AV2 file.

In order to utilize the gamma data contained in the AV2 file for characterizing LLCE contamination, certain data for the LLCE itself must be available, along with data on the tank waste. This data is obtained using the LLCEDATA program. Refer to the companion manual, LLCEDATA and LLCECALC for Windows Version 1.0, Volume I: User's Manual, for details on the use of the LLCEDATA program. The user selects the proper equipment data by identifying its unique drawing and part numbers in the database. This data include certain critical dimensions (such as the distance from thermocouple tip-to-mounting flange), the mass of the equipment, and the geometry correction factors.

Only one set of geometry correction factors exists at present, and it is appropriate for all positions along the length of equipment where there is sufficiently little mass per unit length so that self-absorption of ${ }^{137} \mathrm{Cs}\left({ }^{137 \mathrm{~m}} \mathrm{Ba}\right)$ gamma rays is negligible. The user should verify this assumption for a specific LLCE before proceeding to use the default values of the correction factors. The present geometry factors were produced by the Fluor Daniel Hanford, Inc., Nuclear Physics and Shielding group and are based on Monte Carlo simulations of the LLCE measurement (Troyer et al. 1998). Future LLCE retrievals will likely include equipment for which these assumptions are not correct, and new geometry correction factors will be required. An example is a pump. This type of equipment is massive in the vicinity of the pump housing and may contain quantities of contaminant inside that cannot be removed by retrieva!. It may be necessary in such cases to make assumptions about the location of the contamination before geometry correction factors can be computed. It would be conservative, for example, to assume the contamination is all inside the pump. At other locations on this equipment that consist of connecting pipes, the mass per unit length can be small, and the default geometry factors may be valid. In any case, for such equipment, the user must examine the geometry correction factor problem and then input new values where appropriate. The Nuclear Physics and Shielding group should be consulted to obtain new geometry correction factors. LLCEDATA allows the user to input new geometry correction factors. These factors can be specified as variable along the LLCE to accommodate cases where diameter and attenuation effects are length dependent. 
If the geometry of the gamma measurement equipment is changed (detector distance from LLCE axis and shieiding dimensions) to accommodate larger LLCE, then new geometry correction factors are required. Again, the user must verify that appropriate geometry factors are being used by LLCECALC.

The present geometry correction factors are 1.481 for detector No. 1, 1.936 for detector No. 2, and 1.9605 for detector No. 3. All are greater than unity because the major function of this factor is to convert the point source detector calibration, for which the detectors are more efficient, to a line source calibration, for which the detectors are less efficient. The factors are larger for detectors 2 and 3 because of the effectively larger sampling length along the line source for these shielded detectors.

After selecting the equipment data, the LLCEDATA user next selects the tank waste data for the tank from which the LLCE is being retrieved. There are options in the program that allow the user to edit both the equipment and the tank waste data. The user can also add to the tank waste data when necessary. When these data are successfully selected and edited, the user saves the data in a unique file designated the *.EDF file. The EDF and the AV2 file are used by the program LLCECALC in performing the ${ }^{137} \mathrm{Cs}$ activity analysis for the LLCE and in characterizing its total contamination.

The total ${ }^{137} \mathrm{Cs}$ activity on the LLCE following retrieval and washing is computed by LLCECALC by using both the EDF and AV2 file to assign ${ }^{137} \mathrm{Cs}$ activity to each 1 -ft length of the LLCE. The process is complicated because of the many possible conditions encountered in the actual practice of LLCE retrieval and gamma measurement. Section 2.3 explains in detail each of these conditions and the methodology that is used.

These conditions include the possibility that detectors will fail, and they do; this creates gaps in the gamma data along the length of the LLCE. Sometimes the gamma data does not include the entire length of the LLCE for which contamination is expected; e.g., the tip-to-mounting flange for a thermocouple tree is larger than the recorded data. This condition is not easily detected from the AV2 file because the records are associated with crane position and are not associated with the position along the length of the LLCE. The process of relating crane position to position on the LLCE requires user input and LLCECALC prompts the user for required information at the appropriate stage in the analysis. There are other instances where the user is given the option to input information to assist the analysis, such as in the process of interpolating values for missing data where gaps are detected in the AV2 file.

There are frequent reversals in the motion of the crane as the LLCE is moved back into the tank, usually to return to a position where activity is high and another wash might remove more of the contamination. These motion reversals greatly complicate the software analysis of the LLCE for total ${ }^{137} \mathrm{Cs}$ activity based on data contained in the AV2 file. LLCECALC must detect the reversals, determine the length extent of each one, and then update the gamma activities for the portion of the LLCE length where reversals have occurred to be the last measurements for that length range. Section 2.3 .4 discusses this process.

Normally, gamma spectral data from three detectors are available for each 1-ft interval along the length of the LLCE. However, sometimes one or more of the detectors are not operable at a given position. This condition can be caused by equipment malfunction and also by activities that are too low or too high to be registered by all detectors. When data are available from two or more detectors at a given position, the LLCECALC program will compute a statistically weighted average activity. The statistical weighting factor for each detector is the reciprocal of the square of the $\%$ error in the activity. This weighting method has the effect of placing more credence on detector results at a given position having smaller \% errors. When data are present for all three detectors, detector 1 is typically given the most weight because it is the most efficient and will have the smallest \% error. Appendix B outlines the AV2 format and discusses the statistically weighted average in more detail.

Because of a difficulty in properly calibrating detectors No. 2 and No. 3 for their counting efficiency with available point sources, LLCECALC must determine a relative efficiency for these two detectors based on a comparison to detector No. 1 activities. It is assumed that detector No. 1 is properly calibrated so that intervals along the length of the LLCE for which gamma data are present for all three detectors are used to compute an average normalization factor for detectors No. 2 and No. 3. These are called 
shielding correction factors in the program dialogue presented to the user as the analysis proceeds. There are default values of these shielding factors in the program as determined either by computer simulation or by a similar comparison to detector No. 1 by others during previous hand calculations. However, they are not used because systematic differences are observed between detectors No. 1 , No. 2 , and No. 3 for the same positions, indicating these defaults are in error. LLCECALC always computes its own shielding factors for detectors No. 2 and No. 3 from the actual gamma data contained in the AV2 file.

The current version of the LLCECALC program operation will take a preference for the values of the shielding factors by first looking in the Ilcecalc.ini file. If LLCECALC finds shielding factors in the ini file, it will use the ini values of the shielding factors and not auto-calculate them. If the ini file does not have the values, or they are commented, then LLCECALC will auto-calculate the shielding factors. By default, the Ilcecalc.ini file has the shielding factors commented, implying the program will auto-calculate shielding factors by default.

\subsection{GAMMA ASSAY FILE DATA ANALYSIS PROCESS}

The ilgorithms that implement the methodology used when LLCECALC processes an AV2 file are discussed $r_{i}: x t$. The reader should also refer to the companion document, LLCEDATA and LLCECALC for Windows Version 1.0, Volume $l$ : User's Manual, for instructions on carrying out this analysis with LLCEDATA and LLCECALC.

The window displayed below, Analyze Gamma Assay Data, is the on-screen window displayed when LLCECALC is preparing to read the AV2 file. At the successful conclusion of this step, the Characterization button becomes active, and if selected, the procedure outlined in Section 2.4 is performed. 


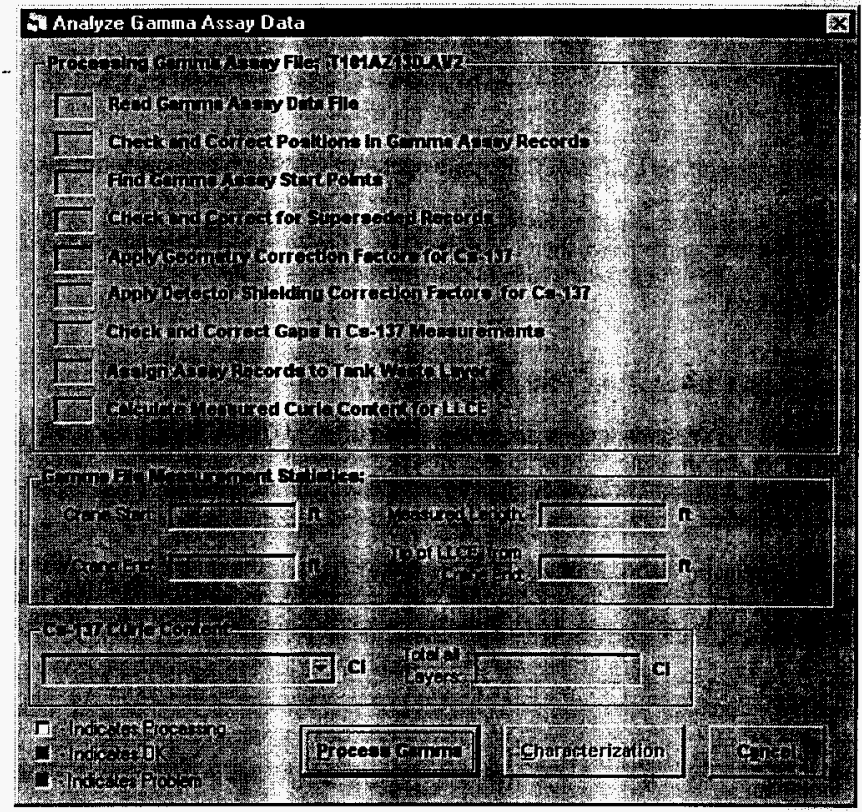

After the selection of Process Gamma is made, the nine steps terminate with the determination of the amount of ${ }^{137} \mathrm{Cs}$ that remains on an LLCE after retrieval. The window shown has the following options:

Process Gamma

Stop Process

Cs-137 Curie Content Click on the down arrow button at the right of this field to view the ${ }^{137} \mathrm{Cs}$

Characterization curie content of each of the waste layers. This option is only available after step 9, Calculate Measured Curie Content for LLCE, is complete.

Click this button to initiate processing of the currently selected AV2 file. The specific AV2 file name is shown at the top of this window.

This button replaces the Process Gamma button once processing has begun. If desired, click this button to stop processing at the end of the current step. It is possible to view the status report at this point. Once it has been clicked, it is again replaced by the Process Gamma button.

Click this button to open the LLCE Waste Characterization

Calculations window and initiate the characterization of the LLCE waste of the data files, discussed in Section 2.4. This button can only be clicked after step 9, Calculating Measured Curie Content for LLCE, is complete. 
Cancel
Click this button to close the Analyze Gamma Assay Data window. This button is only active when the Process Gamma button is visible, when the program is idle.

The Analyze Gamma Assay Data window has three main sections. The first section, titled Processing Gamma Assay File, lists the nine main steps that are performed as the AV2 file is analyzed. Colored buttons are used to indicate the current status as the program completes each step of the process. The boxes to the left of each step change color from grey (idle) to yellow (in process) to bright green if the step is completed successfully or red if there was error. If an error occurs, processing of the AV2 stops at the step where the error occurred.

The second section of the window, titled Gamma File Measurement Statistics, provides crane position information read from the AV2 file. The Crane Start position; Crane End position; and the total crane travel, Measured Length, are shown. The distance from the bottom tip of the LLCE relative to the end crane position, Tip of LLCE from Crane End, is also shown.

The third section, titled Cs-137 Curie Content, displays the measured ${ }^{137} \mathrm{Cs}$ content on the LLCE for each tank waste layer. The user must click on the down arrow to view the ${ }^{137} \mathrm{Cs}$ content for each layer that the LLCE was pulled from. The Total ${ }^{137} \mathrm{Cs}$ content of the LLCE is also shown.

The following nine sections describe in detail each of the processing steps performed by LLCECALC when the Process Gamma button is selected in the Analyze Gamma Assay Data window.

\subsubsection{Read Gamma Assay Data File}

The AV2 file specified at program startup is read line by line, and the information is extracted and stored in the Rawdata table of the EDF database file. The AV2 file format is documented in Appendix B. The format of the Rawdata table is found in Appendix A4, Section A4.3.0. The following eight steps outline the procedure used when reading the AV2 file.

1) The AV2 file is opened for reading.

2) The Rawdata table is opened in the EDF database file.

3) The Rawdata table is purged to make sure it is empty.

4) The AV2 file is read line by line until the first character read in the current line is the letter 'A.' This indicates the beginning of a gamma assay record in the AV2 file.

5) The current line contains the spectrum record ID number, the date of record acquisition, the start time for data acquisition, the count time in seconds, the crane date and time, and the crane position at the end time for the data acquisition.

6) Additional lines are read from the AV2 file until the beginning of a new gamma assay record is detected. These additional lines contain the nuclide atomic number, nuclide description, the activity in microcuries, and the indicated percent error in measurement of the measured nuclides of the gamma assay record. Information read in step 5 is combined with the information read in this step to generate a record in the Rawdata table.

7) When the beginning of a new gamma assay record is detected in step 6 , processing goes back to step 5 . This continues until the entire AV2 file has been read.

8) The number of records generated in the Rawdata table is shown in parentheses after the line Read Gamma Assay Data File in the first section of the Analyze Gamma Assay Data window. 


\subsubsection{Check and Correct Positions in Gamma Assay Records}

2.3.2.1 Feet and Curie Corrections. Now that the uncorrected AV2 data records have been read, the first part of this task is to ensure that the crane position measurements are positive and that the untts of measurement are in feet. Also, the activities are converted from microcuries to curies.

1) The Rawdata table, created in Section 2.3.1, is copied to the Gammadata table. Both tables are in the EDF database file. All further processing of the gamma assay data is done by manipulating the content of the Gammadata table. The Rawdata table is left unchanged to document the as-read content of the AV2 gamma assay file. The format of the Gammadata table is found in Appendix A4, Section A4.3.0, and is identical to the Rawdata table.

2) The Gammadata table is opened and sorted by the [Position] data field. Since positions may be negative or positive, the absolute value of the crane position of the first and last records are examined. If either of these crane position values are greater than 1000 , LLCECALC assumes that the crane position measurements were recorded in thousandths of a foot. Otherwise it is assumed that the crane position measurements were recorded in feet.

3) All the records in the Gammadata table are read. For each record the following steps are performed.

i) If the crane position measurements are in thousandths of a foot, the crane position is divided by 1000 to convert crane positions to feet. The absolute value of the crane position is taken to ensure the crane positions are positive. The updated crane positions are saved to the [Position] data field in the Gammadata table.

ii) The activity in each record is divide by $1.0 E+06$ to convert the activity from microcuries to curies. The updated activity values are saved to the [Activity] data field in the Gammadata table.

iii) The gamma assay system detector number is extracted from the record number. The detector number $(1,2$, or 3$)$ is the number that directly follows the letter ' $A$ ' in the gamma assay record ID. The detector number is saved to the [Detector] data field in the Gammadata table.

iv) The record ID is truncated by saving the last seven characters of the record ID to the [Record] data field in the Gammadata table. This converts the record ID to a seven-digit number.

2.3.2.2 Zero Crane Positions. There are occasions, due to anomalies with the AV2 file creation process, when the crane positions are recorded as zero. To correct for the problem where zero crane positions were recorded for some of the data in a gamma assay record, the following algorithm is used.

1) The Gammadata table is reopened.

2) First, a check is made to ensure that all of the crane positions recorded for a unique gamma assay record ID have uniform crane position values.

All of the records in the Gammadata table are read. For all records that have the same record ID, the crane position measurements are examined. If the crane positions are not the same, the crane positions for that record ID are all set to the average of the crane positions found for that record ID. 
An exception to this procedure is when there are zero and nonzero crane position measurements found for the same record ID. In this case all crane positions are set to the average of the nonzero crane positions found for that record ID.

3) Now that all of the nonzero crane positions are uniform for each record ID, the Gammadata table can be reopened to look for and correct the zero crane position problem.

4) All of the records in the Gammadata table are read. For all records that have the same record ID number, the crane position measurement is examined. If all of the crane positions for a record ID are zero, the user is prompted by the Enter Missing Crane Position window, shown below, to enter the missing position information.

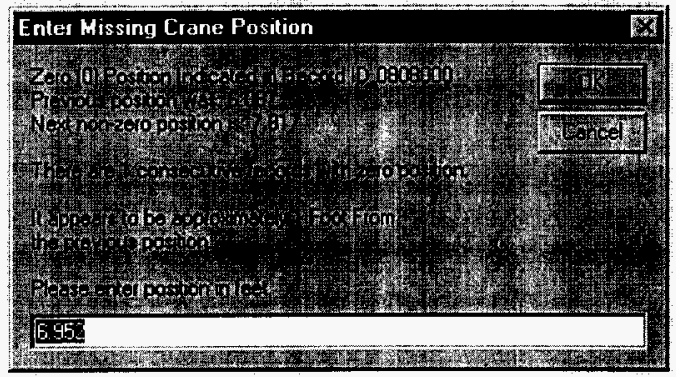

5) To assist the user when making this decision, the program displays the previous and next nonzero crane position. The location that is midpoint between these two positions is displayed as the default entry. The program user can accept the default value or enter a new value.

The example window shown above, Enter Missing Crane Position, indicates that " 1 consecutive" zero was identified. If LLCECALC finds more than one consecutive zero in the AV2 file, this window will indicate the number of consecutive zeros. The program will estimate the zero positions by noting the previous and next nonzero positions. Already knowing the number of zeros positions, LLCECALC will linearly interpolate between values before and after the spacing to approximate an even distribution for the user to enter for the missing crane positions. This is demonstrated by the c1_1layer.AV2 example in Section 8.0 of the user's manual.

If the calculated midpoint is approximately one foot from the previous and next nonzero position, the program displays this fact in the dialog box. A value greater than 0.75 and less than 1.25 is considered "approximately 1 Foot." Since the crane positions between two consecutive record IDs should be approximately one foot apart, this indicates that the default position is close to being correct.

\subsubsection{Find Gamma Assay Start Points}

The purpose of this step is to determine the crane position at the beginning of a gamma assay acquisition record. The AV2 file only records the position of the crane when a current data acquisition is completed. This position represents the end crane position for a given record. The activity reported in a gamma assay record is the total activity measured for the length of the LLCE between the crane start and 
crane end positions. Because the crane start position is not recorded in the AV2 file, it must be calculated by LLCECALC as follows.

1) The Gammadata table is opened.

2) The Positions table is opened. The Positions table is stored in the EDF database file. A description of the Positions table can be found in Appendix A4, Section A4.10.0.

3) The Positions table is purged to make sure it is empty.

4) All of the records in the Gammadata table are read. For each unique record ID in the Gammadata table, a new record is created in the Positions table. All of the records in the Gammadata table, with the same record ID number, are read to provide information to fill in data fields in the Positions table. The Position table data fields are filled as follows.

i) Set Positions [Record] data field to be equal with the Gammadata [Record] data field.

ii) Set Positions [End] data field to be equal with the Gammadata [Position] data field.

iii) Set Positions [Start] data field equal to -1 , implying that it is not yet defined.

iv) Set Positions [137CS] data field to be TRUE, if there was a ${ }^{137} \mathrm{Cs}$ record ID read in Gammadata table.

v) Set Positions [Time] data field equal to the Gammadata [Time] data field.

5) The Positions table is reopened.

6) The Positions table records are examined to calculate the minimum (MIN), maximum (MAX), and average (AVERAGE) crane travel for all sets of records that are sequential and have a travel distance close to one foot. Records are considered sequential if their record ID numbers differ by 10 . A travel distance is considered close to one foot if it is greater than $0.8 \mathrm{ft}$ and less than $1.5 \mathrm{ft}$.

7) Starting with the first record in the Positions table, all records are read to determine where multiple gamma assay records exist for the same crane position. This occurs when the crane remains stationary for 2 minutes or more. All records that have the same position are deleted except for the last record made at that crane position.

If the [End] data field of the current record is equal to the [End] data field of the previously read record, the previous record is deleted from both the Positions and Gammadata tables.

8) Now the crane start positions can be calculated. The following steps are performed to calculate the crane start positions.

i) Records in the Positions table are read starting with the first record.

ii) LLCECALC assumes that for the first record the start crane position is equal to the [End] data field value minus the average travel (AVERAGE) calculated in step 6.

iii) The gap (GAP) is calculated between the current and previous records by dividing the difference between these record ID numbers by 10 . Because the 
sequential record ID number will increase by 10 , the result of this division is 1 when there is no gap.

iv) The difference (DIFF) is calculated by subtracting the [End] data field (END̄1) of the previous record from the [End] data field (END2) of the current record.

v) The following algorithm determines the crane start position for the current record in the Positions table.

If GAP > 1, implying a missing record ID, then:

If DIFF > MAX, then there is at least one missing record between this current record and the previous record. In this case, the [Start] data field is set equal to END2 - AVERAGE.

If $0<$ DIFF $<$ MAX, it is assumed that although there is a gap in record ID numbers, there is no real gap between the current record and the previous record. In this case, the [Start] data field is set equal to END1, which is the end point of the previous record.

If DIFF $<0$, this indicates that the crane moved in the reverse direction; this detects the backward motion of the crane. In this case, the [Start] data field is set equal to -99 . The -99 acts as a flag to indicate that backward motion was detected for this record.

IF GAP $=1$, implying no record IDs are missing, then:

If DIFF > 0 , then the [Start] data field is set equal to END1, which is the end point of the previous record.

If DIFF $<0$, this indicates that the crane moved in the reverse direction, backward motion detected. In this case, the [Start] data field is set equal to -99 . The number -99 acts as a flag to indicate that backward motion was detected for this record.

9) The start point for each record is calculated as outlined in step 7 and saved to the [Start] data field in the Positions table.

\subsubsection{Check and Correct for Superseded Records}

When the crane reverses direction to allow for rerinsing of a section of the LLCE, there may be gamma assay records that contain data for previously measured sections of the LLCE. Since the LLCE is being rerinsed, and contamination is being reduced on the LLCE surface, these new gamma assay records should replace the previous records for these already measured sections of the LLCE. This section of the code determines which gamma assay records need to be deleted or revised because they are superseded by newer gamma assay records. The algorithm is as follows.

1) The Positions table is opened in reverse order.

2) The Positions table records are read until the [Start] data field is found equal to -99 or the end of file is encountered. If all of the records are read without encountering the [Start] data field equal to -99 , then there are no superseded records. 
3) If the [Start] data field value is found to be -99 , then the following steps are performed.

i) Set the variable START to be equal with the current record [Start] data field value.

ii) Set the variable END to be equal with the current record [End] data field value.

iii) Delete the current record, with the number -99 in the [Start] data field, in both the Positions and Gammadata tables.

iv) Continue to read additional records in the Positions table. Delete the current record in both the Positions and Gammadata tables until END is greater than or equal to the current record [Start] data field value and the current record [Start] data field value is not equal to -99 .

v) When the above condition is met, the following actions are performed.

If the current record [Start] data field is equal to END, then the current record is deleted in the Positions and Gammadata tables. This corrects the instance of backward motion. Read the next record in the Positions table and go back to step 2).

If END is greater than the current record [Start] data field and END is also less than the current [End] record data field, then the current record [End] data field is set equal to END. Activities for all records in the Gammadata table will be reduced since overall measured length of this record is reduced by changing the end point. The reduction is calculated as follows.

Let the variable Origstart be set equal to the current [Start] record data field value from the Positions table.

Let the variable OrigEnd be set equal to the current [End] record data field value from the Positions table.

Let the variable NewEnd be set equal to the END value from part ii of step 3 above.

Let the variable OldActivity be set equal to the current [Activity] record data field value. The new activity is then calculated as follows:

$$
\text { NewActivity }=\frac{(\text { NewEnd }- \text { OrigStart })}{(\text { OrigEnd }- \text { OrigStart })} \times(\text { OldActivity })
$$

The value calculated for NewActivity is saved to the [Activity] data field of the current record in the Gammadata table.

4) Read the next record in the Positions table, and go back to step 2. This process will continue until all records have been read.

\subsubsection{Applying Geometry Correction Factors for ${ }^{137} \mathrm{Cs}$}

Geometry factors are applied to the activities by multiplying the measured ${ }^{137} \mathrm{Cs}$ activity in the gamma assay records by the geometry factor. Because the value of these factors are related to the cross-sectional shape of an LLCE, the geometry factors will change when there are changes in the 
cross-sectional shape of the LLCE. Therefore, it is essential that the gamma assay records be matched to the actual position on the LLCE so the appropriate geometry correction factors are applied. See Section 1.3.1 for a discussion on the imposed limitations of this methodology.

1) The Gammadata table is opened with the records sorted by the [Time] and [Position] data fields.

2) The Positions table is opened with the records sorted by the [Record] data field.

3) The value MIN is set equal to the [Start] data field of the first record in the Positions table.

4) The value MAX is set equal to the [End] data field of the last record in the Positions table.

5) The measure length is then calculated as follows:

\section{MEASURED LENGTH $=$ MAX - MIN .}

6) The next step is to determine how the crane positions recorded in the gamma assay records relate to the position on the LLCE. The user is prompted by the Decision Point Relate Crane Position to LLCE window, shown below, to specify this relation.

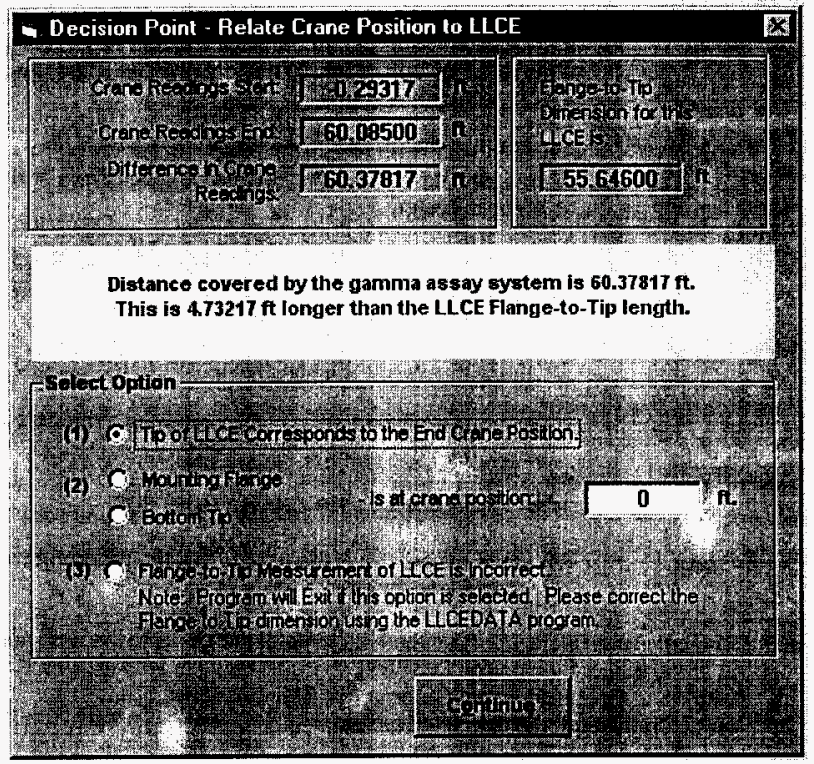

There are three main sections in this window. The top section provides the user with information on the crane measurements and the flange-to-tip dimension of the LLCE. This information comes from the variables discussed above: 


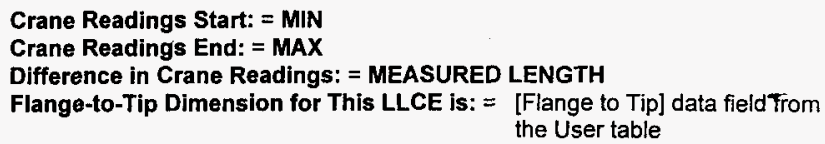

The User table is stored in the EDF database file. A description of the User table can be found in Appendix A4, Section A4.15.0.

The middle section of the window compares the variable MEASURED LENGTH to the LLCE flange-to-tip dimension, FLANGE2TIP. Because FLANGE2TIP represents the length of the LLCEE exposed to tank waste, MEASURED LENGTH should be larger than FLANGE2TIP. The difference between MEASURED LENGTH and FLANGE2TIP is displayed in this middle section. A warning message is displayed if MEASURED LENGTH is less than FLANGE2TIP.

The bottom section of the window contains three options for the user. The first two options allow the user to define the variable DISTANCE. The variable DISTANCE is the distance from the bottom tip of the LLCE to the detectors when the crane is at the end crane position, MAX. The three options perform the following tasks.

Option (1) The first option specifies that the bottom tip of the LLCE corresponds to the last gamma assay record, which would be the last crane position. This is the default option. If this option is selected, then the variable DISTANCE is set to 0 .

Option (2) The second option allows the user to enter the crane position corresponding to when the LLCE mounting flange or the LLCE bottom tip is at the detector.

If the crane position, FLANGE CRANE POSITION, for the LLCE mounting flange is entered, then the variable DISTANCE is calculated to be:

\section{DISTANCE = MAX - FLANGE2TIP - FLANGE CRANE POSITION}

If the crane position for the LLCE bottom tip. TIP CRANE POSITION, is entered, then the variable DISTANCE is calculated to be:

\section{DISTANCE $=$ MAX - TIP CRANE POSITION}

Option (3) The third option allows the user to exit the program if the flange-to-tip dimension is incorrect.

7) The calculated distance, from the bottom tip of the LLCE to the detectors when the crane is at the end crane position DISTANCE, is displayed in the Analyze Gamma Assay Data window in the section titled Gamma File Measurement Statistics in the Tip of LLCE from Crane End: field, in units of feet.

8) The crane positions in the Gammadata and Positions tables are modified so that the tip of the LLCE corresponds to the position 0.0 . This procedure is accomplished by the following methodology.

i) The Gammadata table is opened, each record is read, and the [Position] data field is modified. The [Position] data field is set equal to MAX - DISTANCE current [Position] data field value. 
The Positions table is opened, each record is read, and the [Start] and [End] data fields are modified. The [Start] data field is set equal to MAX - DISTANCE - the current [End] data field value. The [End] data field is set equal to MAX DISTANCE - the current [Start] data field value.

9) A check is made to see if the tip of the LLCE, at the 0.0 position, falls in the middle of an existing gamma assay record. If the tip of the LLCE is in the middle of an existing record, the [Start] data field in the Positions table for this record ID is set to zero so that the start point corresponds to the tip of the LLCE $(0.0)$. The activities for this record ID in the Gammadata table are adjusted proportionally to the change in the distance traveled for this record ID. The same method described in Section 2.3.2, step 3, part v, is used for reducing the activities.

10) Now that the 0.0 position represents the tip of the LLCE, a check is made to determine if there are records that fall below the tip of the LLCE or extend above the mounting flange of the LLCE.

If the mounting flange falls in the middle of an existing record, the [End] data field of this record is set to FLANGE2TIP. The activities for this record ID in the Gammadata table are reduced proportionally to the change in the distance traveled for this record ID. The same method described in Section 2.3.4, step 3, part $v$, is used for reducing the activities.

All records that are below the tip of the LLCE or above the mounting flange of the LLCE are deleted from both the Positions and Gammadata tables. A record is deleted if the [End] data field value is less than zero, indicating the record is below the tip, or if the record's [Start] data field value is greater than FLANGE2TIP, indicating the record is above the mounting flange.

11) A check is made to determine if the gamma assay measurements cover the full length of the LLCE exposed to the tank waste. The Positions table is reopened with the records sorted by record ID number in descending order.

The first record in the Positions table is examined. If the [Start] data field is greater than zero, then there is a gap between the tip of the LLCE $(0.0)$ and the start point of this first record. A new record is created in the Positions table to define this gap with its [Start] data field set to 0.0 and its [End] data field set to the start point for the first record.

The last record in the Positions table is examined next. If the [End] data field value is less than FLANGE2TIP, then there is a gap between the end of the last record and the mounting flange of the LLCE. A new record is created in the Positions table to define this gap. The new records [Start] data field is set to the [End] data field of the last record, and the new records [End] data field value is set to FLANGE2TIP.

12) The LLCEGeom table is opened. This table contains the geometry factors for the selected LLCE. The LLCEGeom table is stored in the EDF database file. A description of the LLCEGeom table can be found in Appendix A4, Section A4.7.0.

Geometry factors are applied by multiplying the measured ${ }^{137} \mathrm{Cs}$ activity in the gamma assay records by the factors. The geometry factor for each detector is stored in a set that is defined for a specific location along the length of the LLCE. There may be several sets of geometry factors defined for one LLCE in order to cover the entire length of the LLCE. Each set of these geometry factors is defined with start and end locations, which indicate where the factors apply along the length of the LLCE. These locations are measured from the tip of the LLCE. 
13) If the [Start] and [End] point of the Gammadata data record is found to fall between two sets of geometry factors, the following steps are followed.

i) The [Position] data field in the Gammadata data table is set equal to the position location between the two sets of geometry factors. The corresponding data record in the Positions data table is opened, and its [End] data field is set equal to the position location between the two sets of geometry factors.

ii) The activity in the original Gammadata data record is reduced by an amount proportional to the reduced length of the assay record. The remaining activity is calculated by subtracting the reduced activity from the original activity value.

iii) A new data record is created in the Gammadata database table with all data fields set to the value of the previous Gammadata data record. The data record ID is equal to the original record ID number +5 . The [Position] data field is set equal to the value of the [Position] data field of the original Gammadata data record before it was changed (shortened). The [Activity] data field is set equal to the value of the remaining activity calculated in the previous step (ii).

iv) A new data record is created in the Positions database table with the [Start] data field set equal to the geometry factors boundary position. The [End] data field is set equal to the [Position] data field in the previous step. The [Record] data field is set equal to the record ID number determined in the previous step (iii).

14) The Gammadata table is opened, and only the ${ }^{137} \mathrm{Cs}$ records are read. For each ${ }^{137} \mathrm{Cs}$ record in the Gammadata table, the [End] data field value is examined to determine which set of geometry factors is applied to the ${ }^{137} \mathrm{Cs}$ activity. The appropriate geometry factor, based on the detector number from the ${ }^{137} \mathrm{Cs}$ record, is applied to the data field [Activity] of that record.

This continues until all ${ }^{137} \mathrm{Cs}$ records have been read in the Gammadata table and geometry factors have been applied to all the ${ }^{137} \mathrm{Cs}$ activities.

\subsubsection{Applying Detector Shielding Correction Factors for ${ }^{137} \mathrm{Cs}$}

Shielding correction factors are applied by multiplying the measured ${ }^{137} \mathrm{Cs}$ activity in the gamma assay records by the correction factor. Each detector has a correction factor that is applied to the measured ${ }^{137} \mathrm{Cs}$ activity for that detector. These correction factors are based on the shielding design for the detectors, so they should remain constant for an LLCE. Currently, however, this is not possible; see Section 1.4 .2 for a discussion of these factors.

The shielding correction factors that have been calculated for the current detector shield design are defined in the l/cecalc.ini file. The shielding correction factors may be read from this file if the user desires. By default, the program calculates its own shielding correction factors based on the ${ }^{137} \mathrm{Cs}$ activities for all three detectors. The shielding correction factors in the Ilcecalc.ini file are only used if the user uncomments the factors in the file. By default, the factors are commented in the INI file, implying that the program is to auto-calculate the correction factors. A description of the /lcecalc.ini file can be found in Appendix A3, Section A3.1.0.

1) The program assumes default values for the shielding correction factors based on current detector shield design as follows:

Detector \#1 $=1.0$

Detector \#2 $=1.53$

Detector $\# 3=2.95 \mathrm{E}+07$. 
These default values are assumed by the program in case the /lcecalc.ini file does not define these values.

2) The Ilcecalc.ini file is opened, and values for the shieiding correction factors are read for all three detectors if they are defined in this file. Shielding correction values read from this file will replace the default values described in step 1.

3) The correction factor for detector No.1 is always assumed to be 1. The correction factors for detectors No. 2 and No. 3 are estimated by the program and compared to the current shieiding factors as specified by the default or INI file. The following algorithm is used to estimate these factors. For any of the steps outlined below, if a detector activity is negative (an anomaly that has been observed in AV2 files), the activity is ignored.

i) The Gammadata table is opened for all ${ }^{137} \mathrm{Cs}$ records and sorted by end crane position.

ii) Initialize the following six values:

$$
\text { Sum21 }=\text { Count21 }=\text { Sum31 }=\text { Count31 }=\text { Sum32 }=\text { Count32 }=0 \text {. }
$$

iii) The ${ }^{137} \mathrm{Cs}$ records with the same record ID number are read. There will be no more than one ${ }^{137} \mathrm{Cs}$ record for each of the three detectors for the one record ID.

For this record ID:

If there is activity for detectors 1 and 2, then add to perform the sum:

$$
\begin{aligned}
& \text { Sum21 }=\text { Sum21 }+ \text { Activity Detector } 1 / \text { Activity Detector } 2 \\
& \text { Count21 }=\text { Count21 }+1
\end{aligned}
$$

If there is activity for detectors 1 and 3 , then add to perform the sum:

$$
\begin{aligned}
& \text { Sum31 }=\text { Sum } 31+\text { Activity Detector } 1 / \text { Activity Detector } 3 \\
& \text { Count31 }=\text { Count31 }+1
\end{aligned}
$$

If there is activity for detectors 2 and 3 , then add to perform the sum:

$$
\begin{aligned}
& \text { Sum32 }=\text { Sum32 }+ \text { Activity Detector } 2 / \text { Activity Detector } 3 \\
& \text { Count } 32=\text { Count } 32+1 .
\end{aligned}
$$

This summation process is repeated until all ${ }^{137} \mathrm{Cs}$ records have been read from the Gammadata table.

iv) Next, the sum and count values are used to calculate shielding correction factors.

For detector No. 2: If Sum21 = 0, this indicates that gamma assay records were not found that had both detector No. 1 and detector No. 2 readings. In this case, Correction Factor 2 is set equal to the current shielding correction factor, from the default or INI file, for detector No. 2.

If Sum21 >0, then the estimated correction factor for detector No. 2 is

\section{Correction Factor 2 = Sum21 $/$ Count21 .}


For detector No. 3: If Sum31 $>0$ then the estimated correction factor for defector No. 3 is Correction Factor $3=$ Sum31 $/$ Count31.

if Sum $31=0$ and Sum32 $>0$, then the estimated correction factor for detector No. 3 is

Correction Factor 3 = (Sum32 $/$ Count32) ${ }^{*}$ Correction Factor 2.

If Sum31 $=0$ and Sum32 $=0$, then Correction Factor 3 is set equal to the current shielding correction factor, from the default or INI file, for detector No. 3.

4) The shielding correction factors can now be applied to the ${ }^{137} \mathrm{Cs}$ records. The Gammadata table is reopened. All ${ }^{137} \mathrm{Cs}$ records are read. If the variable Correction Factor $\mathbf{2}$ is greater than one, then the [Activity] data field for records measured by detector No. 2 are multiplied by Correction Factor 2 . If the variable Correction Factor 3 is greater than one, then the [Activity] data field for the records measured by detector No. 3 are multiplied by Correction Factor 3 .

\subsubsection{Check and Correct Gaps in ${ }^{137}$ Cs Measurements}

This part of the code determines and corrects for gaps in the measured ${ }^{137} \mathrm{Cs}$ activities. Some of the reasons gaps in the ${ }^{137} \mathrm{Cs}$ measurements may occur are as follows.

- $\quad$ Activity is reported for an area of the LLCE, but ${ }^{137} \mathrm{Cs}$ was not one of the isotopes reported for the data acquisition.

- Gamma assay records are missing.

- The gamma assay records do not cover the entire length of the LLCE.

- Equipment or detector problems occurred during LLCE retrieval.

When these gaps occur, they are identified and corrected by the following algorithm.

1) The first step is to extract the ${ }^{137} \mathrm{Cs}$ activity data information from the Gammadata table and store it in the Positions table.

For each record ID in the Positions table, extract the ${ }^{137} \mathrm{Cs}$ activity and percent error data for all three detectors from the Gammadata table. If any activity is less than zero, its value is set to zero. The activity from the Gammadata table for all three detectors is divided by the length of travel for that record. The activities (Ci/ft) and percent errors are stored in the Positions table for that record ID.

2) Next, gaps in ${ }^{137} \mathrm{Cs}$ measurements are determined by the following algorithm.

i) The Positions table is opened, and the records are sorted by the starting crane position.

ii) The [End] data field of the current record and the [Start] data field of the next record are examined. If the current records [End] data field value is equal to the next records [Start] data field value, then there is no gap between the current and next record. 
iii) If the [End] data field of the current record is not equal to the [Start] data field of the next record, a new record is created in the Positions table for the gap. The data fields for the new record are set as follows:

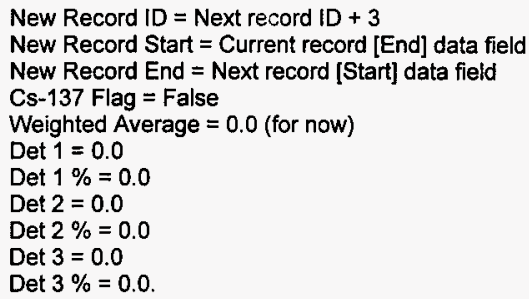

iv) Continue to examine records in the Positions table as outlined above until all records have been examined.

3) The next step examines the Positions table records to look for adjoining records that do not have ${ }^{137} \mathrm{Cs}$ activity reported. If adjoining records are found with no ${ }^{137} \mathrm{Cs}$ activity reported, the records are joined into one record. The following algorithm is followed to combine adjoining data records with no ${ }^{137} \mathrm{Cs}$ activity.

i) The Positions table is reopened and sorted by the start crane position.

ii) The [137CS] data field is examined for the current record. If this data field is false, meaning there is no ${ }^{137} \mathrm{Cs}$ activity, then examining records continues until the next record to be examined has its [137CS] data field value set to true. All intermediate records are deleted between the current data record and the record with ${ }^{137} \mathrm{Cs}$ activity. The [End] data field for the current data record is set to the [End] data field value of the last record deleted. This combines all of the adjoining records without ${ }^{137} \mathrm{Cs}$ activity into the first record.

iii) Examining records continues, looking for and combining adjoining records without ${ }^{137} \mathrm{Cs}$ activity, until all records in the Positions table have been examined.

4) Now that all of the gaps in ${ }^{137} \mathrm{Cs}$ activity are defined, the missing ${ }^{137} \mathrm{Cs}$ activities can be entered.

5) The Positions table is reopened.

6) The [137CS] data field is examined for each record. Where the value of this field is false, the Gap in Gamma Assay Data Found window is displayed, prompting the user to enter the missing ${ }^{137} \mathrm{Cs}$ activity as shown below: 


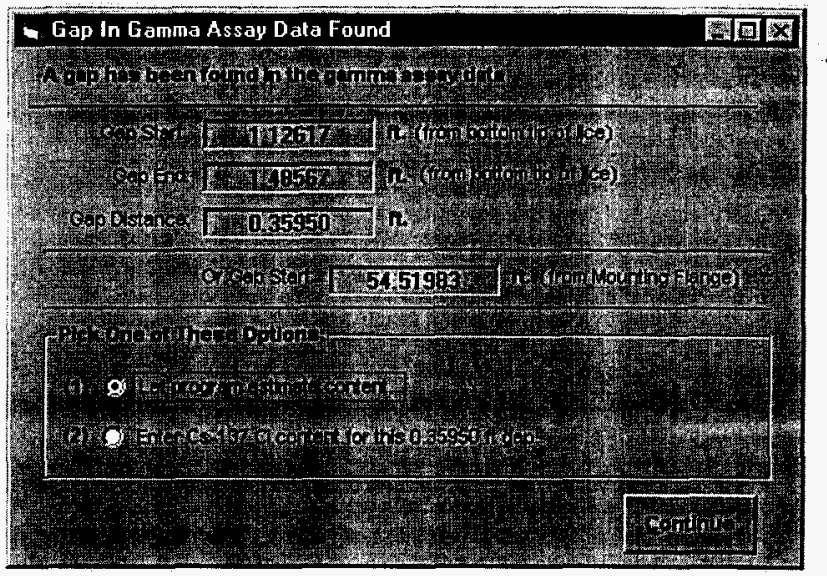

At the top of the this window, the Gap Start, Gap End, and Gap Distance relative to the bottom tip of the LLCE are displayed. The gap start relative to the mounting flange is also displayed.

There are two options the user may select. If the user selects to have LLCECALC estimate the ${ }^{137} \mathrm{Cs}$ content of the gap, the default option, LLCECALC calculates the missing ${ }^{137} \mathrm{Cs}$ content and proceeds to look for the next gap.

The ${ }^{137} \mathrm{Cs}$ content is estimated by linear interpolation between midpoints of the last record's ${ }^{137} \mathrm{Cs}$ average activity and the next record's ${ }^{137} \mathrm{Cs}$ average activity. One exception to this is when the gap is the first record of the Positions table, at bottom tip of LLCE. In this case the activity is made equal to the next record's average ${ }^{137} \mathrm{Cs}$ activity. A second exception to this step is when the gap is the last record of the Positions table, at the mounting flange of LLCE. In this case the activity is made equal to the second to the last record's average ${ }^{137} \mathrm{Cs}$ activity.

If the user selects the second option in this window, then the Enter Missing Gamma Data window appears, prompting the user to enter the missing ${ }^{137} \mathrm{Cs}$ content as shown below: 


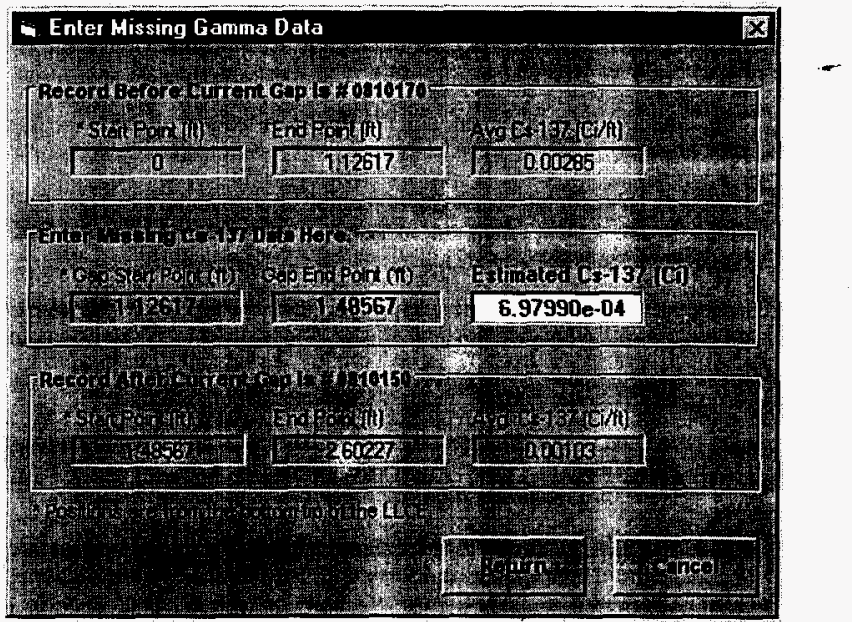

This window shows the start points, end points, and ${ }^{137} \mathrm{Cs}$ activity for the data records before and after the gap. By default, LLCECALC pre-enters the estimate of ${ }^{137} \mathrm{Cs}$ content. The user can change this calculated value, and it will be saved as the ${ }^{137} \mathrm{Cs}$ activity for this gap.

The ${ }^{137} \mathrm{Cs}$ content estimated above, for this gap and record, is saved to the [Det 1] data field with a corresponding $1 \%$ error saved to the [Det $1 \%$ ] data field.

This process continues until all of the data records in the Positions table have been read and all gaps have ${ }^{137} \mathrm{Cs}$ activity defined.

\subsubsection{Assigning Assay Records to Tank Waste Layer}

When an LLCE is retrieved from a waste tank that has more than one defined layer of waste, it is necessary to determine which tank waste layer should be assigned to each gamma assay record. The ${ }^{137} \mathrm{Cs}$ content on the LLCE will be determined for each section of the LLCE that was in a specifically defined tank waste layer.

1) The first step is to determine from the Tankchar table how many tank waste layers are defined in the waste tank. The Tankchar table is opened with the records sorted by tank waste layer. The Tankchar table is stored in the EDF database file. A description of the Tankchar table can be found in Appendix A4, Section A4.14.0.

2) All of the records are examined, and when a new layer number is encountered, the layer number thickness is read from the [Layer Thickness] data field. This current layer thickness is added to the thickness of all previous layers to establish a total thickness for this layer. The total layer thickness is saved to a data array that contains the layer number and total layer thickness. This continues until all records have been examined from the Tankchar table. 
If only one tank waste layer is defined, then the tank waste thickness is set to the LLCE flange-to-tip dimension.

3) The Tankchar table is closed, and the Positions table is opened with its records sorted by start position.

4) If there is only one tank waste layer defined, then layer number one will be assigned to all of the Positions table records. The [Layer Number] data field in the Positions table records will be set to 1 .

If there is more than one tank waste layer, then the following algorithm is followed to determine which tank waste layer should be assigned to the Positions table records.

i) Start with the total layer thickness recorded for layer one.

ii) If the current [End] record data field value is less than the current total layer thickness, then the current layer number is assigned to the [Layer Number] data field for the current record.

iii) Else, if the current records [Start] and [End] data field values are greater than the current waste layer total thickness, then the current tank layer number is incremented, and the new tank layer number is assigned to the [Layer Number] data field for the current record. The current layer thickness is now the total layer thickness for the new tank layer number.

iv) Else, if the current record [End] data field value is greater than the current total layer thickness and current record [Start] data field value is less than the current total layer thickness, then the current record falis between two tank waste layers. In this case the current record [End] data field is set to the current tank waste layer thickness; the end corresponds to the top of the tank waste layer. The current layer number is assigned to the [Layer Number] data field for the modified record. A new record is created with the [Start] data field set to the current tank layer thickness, and the [End] data field is set to the original records end position. The next layer number is assigned to the new record. The ${ }^{137} \mathrm{Cs}$ activities for the new record are the same as the original record.

v) If the current record [End] data field value is greater than the current total layer thickness, then the current layer number is incremented, and the total layer thickness for this new layer is read. The new layer number is assigned to the [Layer Number] data field in the record.

vi) This process is continued until all records in the Positions table have been examined.

\subsubsection{Calculating Measured Curie Content for LLCE}

This is the last step in the gamma assay data analysis process. The ${ }^{137} \mathrm{Cs}$ content of the LLCE is calculated for each tank waste layer and the Total ${ }^{137} \mathrm{Cs}$ content of the LLCE is reported.

1) The Layer table is opened. This table will be used to store the total ${ }^{137} \mathrm{Cs}$ measured for each of the tank waste layers. The Layer table is stored in the EDF database file. A description of the Layer table can be found in Appendix A4, Section A4.4.0.

2) For each defined tank waste layer, the Positions table is opened for a subset of all records in the current tank waste layer sorted by the record [Start] data field. 
3) Each record is examined in this subset of the Positions table. The weighted average of the three detectors is calculated and saved to the [Average] data field in the Positions table.

The statistically weighted average is calculated for each record ID using the following equation (see Appendix B, Section B.2.0, for a discussion of the statistically weighted average):

$$
\text { Average }=\frac{\sum_{i=1}^{3} \text { Act }_{i} \times\left(1 / \text { Err }_{i}\right)^{2}}{\sum_{i=1}^{3}\left(1 / E_{r_{i}}\right)^{2}}
$$

Act $_{\mathbf{i}}$ is the activity for detector \# I

Err $_{\mathbf{i}}$ is the percent error for detector \#

For each record in this subset of the Positions table, the average activity (Ci/ft) is multiplied by the record length ( $\mathrm{ft}$ ) to calculate the ${ }^{137} \mathrm{Cs}$ activity for this record. The calculated activity (Ci) is summed for all Position table records to calculate total ${ }^{137} \mathrm{Cs}$ content for this tank waste layer. The total ${ }^{137} \mathrm{Cs}$ layer content is stored in the Layer table for this layer.

4) The current layer number is incremented, and the process continues from step 2. This continues until all tank waste layers have been processed.

5) The last step is to update the ${ }^{137} \mathrm{Cs}$ content shown in the Analyze Gamma Assay Data window. Total ${ }^{137} \mathrm{Cs}$ content for all tank waste layers is also shown in the window.

The gamma assay data analysis is complete. The Characterize button in the Analyze Gamma Assay Data window now becomes active, and two reports of interest can now be produced. Both reports can be accessed by selecting the File screen menu and then the export option. From this menu, the as-read AV2 file and processed AV2 file data reports can be viewed, exported, or printed by the user.

\subsection{LLCE WASTE CHARACTERIZATION}

After application of the methodology outlined in Section $2.3, a{ }^{137} \mathrm{Cs}$ activity will have been assigned to each $1-\mathrm{ft}$ interval of the LLCE. These activities are simply summed over the length of the LLCE to obtain the total ${ }^{137} \mathrm{Cs}$ activity. The activity for all other radionuclides that contaminate the LLCE are estimated by using the ratios of these radionuclide activities to ${ }^{137} \mathrm{Cs}$ for the layer of tank waste adjacent to each position on the LLCE. LLCECALC assigns a waste layer to each LLCE activity record.

The characterization process consists of comparing the various computed radionuclide activities to limits that affect the encapsulation, transportation, and disposal of the LLCE. The algorithms that implement the actual waste characterization methodology are discussed next. The reader should also refer to the companion manuals, LLCEDATA and LLCECALC for Windows Version 1.0, Volume I: User's Manual, for instructions on carrying out this analysis with LLCEDATA and LLCECALC, and Volume III: Software Verification and Validation. Volume III demonstrates how independent calculations are performed to confirm that both the ${ }^{137} \mathrm{Cs}$ calculations and the resulting characterization are in agreement with the results from LLCECALC. The process gamma and characterization algorithms and methodology discussed in Section 2.3 and this section are verified by independent spreadsheets that confirm the results obtained from LLCECALC to be identical to the independent calculations. The detailed discussions 
presented in Volume III: Software Verification and Validation for both software programs demonstrate independent confirmation of the algorithms used by LLCECALC.

The window displayed below, LLCE Waste Characterization Calculations, is the on-screen window displayed when the LLCE waste is characterized following the AV2 file data analysis process, discussed in Section 2.3, which must be complete before the LLCE waste characterization process can be initiated.

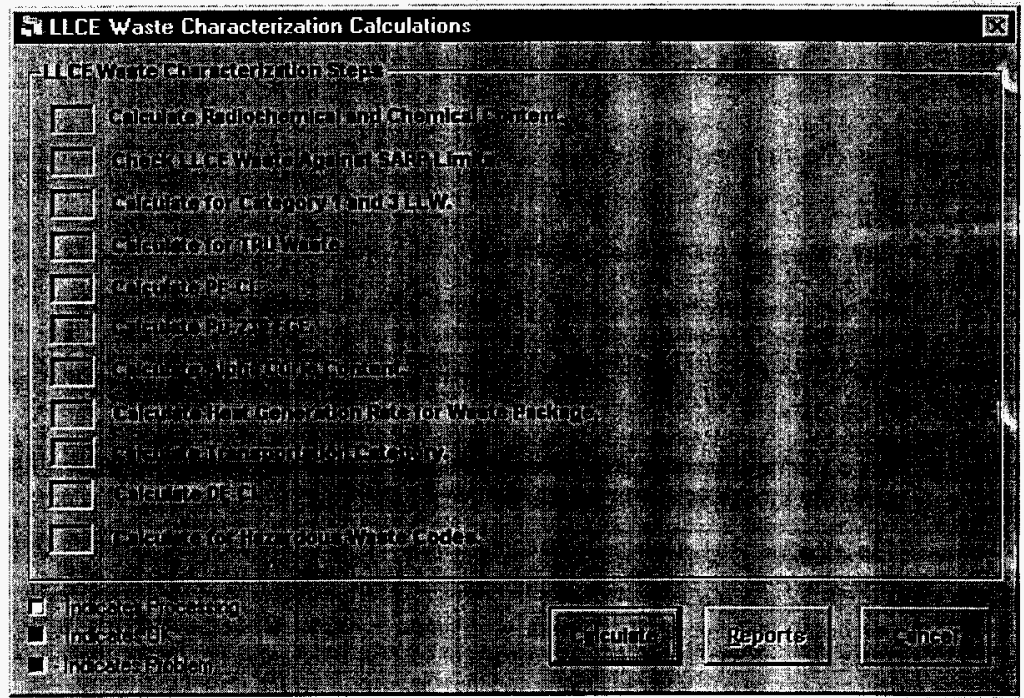

The LLCE Waste Characterization Calculations windows has the following options.

Calculate Click this button to start the calculations necessary to characterize the waste of the processed AV2 file.

Stop This button replaces the Calculate button once calculation has begun. It can be clicked to stop calculation at the end of the current step. Once it has been clicked, it is again replaced by the Calculate button.

Reports Click this button to open the Print Results window and initiate report output. This button can only be clicked after step 11, Calculate for Hazardous Waste Codes, is complete.

Cancel Click this button to close the LLCE Waste Characterization Calculations window. This button is only active when the Calculate button is visible (program is idle).

The LLCE Waste Characterization Calculations window has one main section. This section, titled LLCE Waste Characterization Steps, lists the 11 main steps that are performed as the LLCE waste is characterized. Colored buttons are used to indicate status as the program completes each step of the 
process. Boxes to the left of each step change color from grey (idle) to yellow (in process) to bright green if the step is completed successfully or red if there was error. If an error occurs, the LLCE characterization process stops at the step where the error occurred.

The following 11 sections describe in detail what LLCECALC is doing at each of the processing steps of the LLCE Waste Characterization Calculations window.

The discussion that follows makes reference to the tables in MS Access format that are used by LLCECALC to create the EDF. These data tables are discussed in Section 4.2 and Appendices A1 - A4. The user will find the descriptions of these data tables helpful when interpreting the status report.

\subsubsection{Calculate Radionuclide and Chemical Content}

2.4.1.1 LLCE Waste Mass Determination. The first step in the LLCE characterization process is to determine the LLCE waste mass. The LLCE Waste Mass window is displayed, prompting the user to input the LLCE waste mass. By default the dry clean LLCE waste mass is displayed. The LLCE waste mass can include the mass of the LLCE, the waste film, the flexible receiver bag, and the lifting linkage. The user may edit the LLCE waste mass or accept the default displayed. A sample LLCE Waste Mass window is shown below:

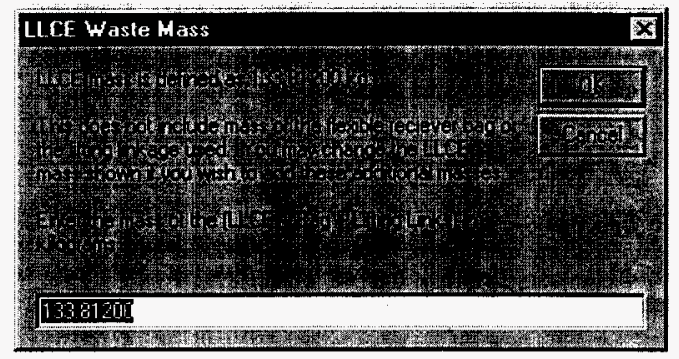

2.4.1.2 LLCE Constituent Content Calculation. The measured ${ }^{137} \mathrm{Cs}$ content of the LLCE is used to calculate the total radionuclide and chemical content of the LLCE waste by ratioing the measured ${ }^{137} \mathrm{Cs}$ content against the established radionuclide and chemical content of the tank. It is assumed that the waste film that remains on the LLCE after retrieval has the same composition as the waste in the tank that the LLCE was in contact with; see Section 1.4.3 for a discussion on this limitation.

1) The Tankchar table is opened for all records where the [Layer Number] data field is equal to 99 . This forms the current record set. Note that layer 99 is the layer generated by LLCECALC to store calculated radionuclide and chemical content of the LLCE waste.

2) The records in the current record set are deleted. This eliminates any previously calculated layer 99 records that may be present in the EDF database file.

3) The Tankchar table is reopened. The units for the content of the radionuclides and chemicals are converted to units of curies (Ci) for the radionuclides and grams (g) for the chemicals.

4) For each waste layer in the tank, the following procedure is followed. 
i) Open the Layer table for the current waste layer. Set CS_LLCE = [Content] data field for the current waste layer. This is the measured ${ }^{137} \bar{C} s$ content on LLCE for the current waste layer.

ii) Reopen the Tankchar table for the current waste layer. Set CS_TANK = [Layer Content] data field, where the [Constituent] data field is equal to ${ }^{137} \mathrm{CS}$ for the current waste layer. This is the established ${ }^{137} \mathrm{Cs}$ content of the current waste layer in the waste tank.

iii) Reopen the Tankchar table for all records in the current waste layer (current record set). For each record (constituent) in the current record set, calculate the LLCE constituent content in the current waste layer by multiplying the tank constituent content (data field [Layer Content]) by CS_LLCE and dividing the result by CS_TANK as shown in the equation below.

$$
\text { LLCEContent }=\frac{(\text { TankContent }) \times\left(C S_{\_} \text {LLCE }\right)}{\text { CS_Tank }}
$$

The result of this calculation, LLCEContent, is the estimated LLCE constituent content from the current tank waste layer. This estimated LLCE constituent content is stored in the data field [Content].

iv) This process is repeated for each waste layer until the LLCE content for each constituent, in each layer, is calculated.

5) The Tankchar table is reopened for ali data records that have distinct data fields [Constituent], [Cas], and [Type]. For each of these distinct records, a new record is created with an assigned layer number of 99 . This will be the layer where the total constituent contents will be summed.

6) An additional set of records is selected from the Tankchar table, consisting of all records except the newly created layer 99 records.

7) Each record is read in the record set created in step 6. The constituent content is summed into the data field [Content] in the corresponding layer 99 record. This is continued until all records have been read. The layer 99 records now contain the estimated radionuclide and chemical content of the LLCE waste.

8) The number of unique constituents in the LLCE waste is reported in the LLCE Waste Characterization Calculations window to provide immediate user feedback. The estimated content of all LLCE constituents is reported in detail in the waste characterization report.

\subsubsection{Check LLCE Waste Against SARP Limits}

The radionuclide curie content of the LLCE waste package, whose calculational methodology is discussed in Section 2.3, is compared against established SARP limits (McCormick 1997) for the LLCE waste container.

1) The Tankchar table is opened, then inner joined to the Radreg table for radiochemical records, where the data field [Layer Number] is equal to 99 . This forms the current record set. 
2) If the curie content of the current record exceeds the SARP (McCormick 1997) limit for _ . that radionuclide, a safety evaluation for packaging is required for this LLCE waste. package. This process is repeated until each record in the current record set has been checked against the appropriate SARP limit.

3) A statement appears in the LLCE Waste Characterization Calculations window indicating if SARP limits have been exceeded. This provides immediate user feedback. These results are also reported in detail in the waste characterization report.

\subsubsection{Calculate for Category 1 and 3 Low-Level Waste (LLW)}

Calculations are done to determine the LLW characterization of the LLCE waste. The LLCE waste is characterized against limits for the following LLW categories:

- $\quad$ Category 1 LLW

- $\quad$ Category 3 LLW

- U.S. Nuclear Regulatory Commission (NRC) Class C

- Interim safety basis (ISB) noncombustible waste limits.

The limits for these categories are specified in HNF-EP-0063-5, Hanford Site Solid Waste Acceptance Criteria (Ellefson 1998), and for NRC Class C, 10 CFR 61.55, "Waste classification."

1) The LLCECont table is opened for the waste container size selected for the LLCE waste package. The container internal volume (ContVol) is set equal to the data field for the appropriate container size. The units of ContVol are cubic meters. The LLCECont table is closed.

2) The Tankchar table is opened, inner joined to the Radreg table for radiochemical records, where the [Layer Number] data field is equal to 99 . This forms the current record set.

3) For each record in the current record set the following procedure is followed.

i) For Category $1 \mathrm{LLW}$ : The curie content $(\mathrm{Ci})$ is divided by ContVol $\left(\mathrm{m}^{3}\right)$ to determine the concentration $\left(\mathrm{Ci} / \mathrm{m}^{3}\right)$ of the current radionuclide in the LLCE waste package. If the Category $1 \mathrm{LLW}$ limit is greater than zero, the concentration is divided by the Category 1 LLW limit, resulting in a fraction value. The summing variable (SUM_Cat1) is set equal to the sum of all the fraction values calculated in this step (i)

ii) For Category 3 LLW: The curie content ( $\mathrm{Ci}$ ) is divided by the container internal volume (ContVol $\left.\mathrm{m}^{3}\right)$ to determine the concentration $\left(\mathrm{Ci} / \mathrm{m}^{3}\right)$ of the current radionuclide in the LLCE waste package. If the Category 3 LLW limit is greater than zero, the concentration is divided by the Category 3 LLW limit, resulting in a fraction value. The summing variable (SUM_Cat3) is set equal to the sum of all the fraction values calculated in this step (ii).

iii) For NRC Class $\mathrm{C}$ : If the value of the data field [sNRC] is true $(-1)$, then the units of the NRC Class $\mathrm{C}$ limit is $\mathrm{nCl} / \mathrm{g}$. In this case the curie content (Ci) is converted to nanocuries (multiply by $1 \mathrm{E}+09 \mathrm{nCi} / \mathrm{Ci}$ ), and the LLCE waste mass $(\mathrm{kg})$ is converted to grams (multiply by $1 \mathrm{E}+03 \mathrm{~g} / \mathrm{kg}$ ). The curie content is then divided by the LLCE waste mass to obtain the concentration $(\mathrm{nCi} / \mathrm{g})$. This concentration is then divided by the NRC class $\mathrm{C}$ limit resulting in a fraction value. 
If the value of the data field [sNRC] is false (0), then the units for the NRC Class $\mathrm{C}$ limit is $\mathrm{Ci} / \mathrm{m}^{3}$. In this case, the LLCE waste mass $(\mathrm{kg})$ is converted to cubic meters (divide by $7840.45 \mathrm{~kg} / \mathrm{m}^{3}$, the mass density for steel) to obtain the LLCE waste volume $\left(\mathrm{m}^{3}\right)$. The curie content $(\mathrm{Ci})$ is then divided by the LLCE waste volume $\left(\mathrm{m}^{3}\right)$ to obtain the concentration $\left(\mathrm{Ci} / \mathrm{m}^{3}\right)$. This concentration is then divided by the NRC class $\mathrm{C}$ limit, resulting in a fraction value.

The summing variable (SUM_NRC) is set equal to the sum of all the fraction values calculated in this step (iii).

iv) For ISB noncombustible: The curie content (Ci) is divided by the container volume (ContVol $\mathrm{m}^{3}$ ) to determine the concentration $\left(\mathrm{Ci} / \mathrm{m}^{3}\right)$ of the current radionuclide in the LLCE waste package. If the ISB noncombustible limit is greater than zero, the concentration is divided by the ISB noncombustible limit, resulting in a fraction value. The summing variable (SUM_ISB) is set equal to the sum of all the fraction values calculated in this step (iv).

4) The four sums calculated in step 3 determine the LLW characterization of the LLCE waste package as follows.

i) If SUM_Cat1 $\leq 1$, the LLCE waste is Category 1 LLW.

ii) If SUM_Cat3 $\leq 1$, the LLCE waste is Category 3 LLW.

iii) If SUM_Cat3 $>1$, the LLCE waste is greater than Category 3 LLW.

iv) If SUM_NRC $\leq 1$, the LLCE waste is NRC class $C$.

v) If SUM_NRC > 1, the LLCE waste is greater than NRC class C.

vi) If SUM_ISB > 1, the LLCE waste requires additional safety analysis.

5) The results of these calculations are reflected in the LLCE Waste Characterization Calculation window to provide immediate user feedback. The results are also reported in detail in the waste characterization report.

\subsubsection{Calculate for TRU Waste}

The LLCE waste is characterized as either Not TRU, Suspect TRU, or TRU waste. TRU waste is, without regard to source or form, waste that is contaminated with alpha-emitting transuranic radionuclides with an atomic number $>92$, with half-lives greater than 20 years, and with concentrations greater than $100 \mathrm{nCi}$ alpha/g of waste at the time of assay. TRU isotopes are identified by footnote $d$ in Table A-2 in HNF-EP-0063-5 (Ellefson 1998).

Suspect TRU is LLCE waste that is contaminated with TRU radionuclides at concentrations somewhat less than $100 \mathrm{nCi} / \mathrm{g}$. The program default will flag any LLCE that is $90 \mathrm{nCi} / \mathrm{g}$ as suspect TRU. The user can change this default by editing the Ilcecalc.ini file, which is currently set to $85 \mathrm{nCi} / \mathrm{g}$. Appendix $\mathrm{A} 3$ documents this ini file.

1) The Tankchar table is opened, inner joined to the LLCENuc table for radiochemical records, where the data field [Layer Number] is equal to 99 and the data field [TRU Isotope] is equal to 1 (true). This forms the current record set.

2) For each record in the current record set, the curie content ( $\mathrm{Ci}$ ) is converted to nanocuries (multiply by $1 \mathrm{E}+09 \mathrm{nCi} / \mathrm{Ci}$ ), and the LLCE waste mass $(\mathrm{kg})$ is converted to grams (multiply 
by $1 E+03 \mathrm{~g} / \mathrm{kg}$ ). The curie content is then divided by the LLCE waste mass to obtain the concentration $(\mathrm{nCi} / \mathrm{g})$. This concentration is then divided by the TRU limit $(100 \mathrm{nCi} / \mathrm{g})$, resulting in a TRU fraction. The TRU sum of fractions (SUM_TRU) for the LLCE waste package is calculated by summing all the TRU fractions calculated in this step.

3) The Ilcecalc.ini file is read. If the suspect TRU level is defined in this file, currently set to $85 \mathrm{nCi} / \mathrm{g}$, it will replace the program default $(90 \mathrm{nCi} / \mathrm{g})$ concentration level for suspect TRU.

4) The sum of fractions (SUM_TRU) calculated in step 2 determines the TRU characterization of the LLCE waste package as follows.

i) If SUM_TRU 21 , the LLCE waste package is TRU.

ii) If SUM_TRU $\geq$ Suspect TRU limit, the LLCE waste package is Suspect TRU.

iii) If SUM_TRU < Suspect TRU limit, the LLCE waste package is Not TRU.

5) The results of these calculations are reflected in the LLCE Waste Characterization Calculation window to provide immediate user feedback. The results are reported in detail in the waste characterization report.

6) If the LLCE waste is identified as Not TRU or Suspect TRU, steps in the LLCE Waste Characterization Calculation window that are applicable only to TRU waste become inactive. The text in the inactive steps will turn grey, and N/A will be displayed beside them. This provides immediate user feedback that these steps are not required. The steps that are only applicable to TRU waste are as follows:

- $\quad$ Calculate PE-Ci

- Calculate ${ }^{239}$ PU FGE

- Calculate alpha curie content.

\subsubsection{Calculate PE-Ci}

If the LLCE waste is TRU as identified in Section 2.4.4, the Plutonium Equivalent Curie (PE-Ci) content is calculated for the LLCE waste package.

1) The Tankchar table is opened, inner joined to the Radreg and LLCENuc tables for radiochemical records, where the data field [Layer Number] is equal to 99 and the data field [PE-Ci Correction Factor] is greater than zero. This forms the current record set.

2) For each record in the current record set the following procedure is followed.

i) The curie content $(\mathrm{Ci})$ is summed with all previous curie contents.

ii) The gram equivalent ( $\mathrm{g}$ ) is calculated by dividing the curie content by the specific activity $(\mathrm{Ci} / \mathrm{g})$. The gram equivalent is summed with all previous gram equivalents.

iii) The $\mathrm{PE}-\mathrm{Ci}(\mathrm{g})$ is calculated by multiplying the gram equivalent by the $\mathrm{PE}-\mathrm{Ci}$ correction factor. The PE-Ci is summed with all previous PE-Cis.

3) The results of these calculations are reflected in the LLCE Waste Characterization Calculation window providing immediate user feedback if the total PE-Ci of the LLCE waste package does or does not exceed the $35 \mathrm{PE}-\mathrm{Ci}$ limit. The total curie content, total 
gram equivalent, and total PE-Ci calculated above are reported in the waste characterization report. If the total PE-Ci exceeds $35 \mathrm{PE}-\mathrm{Ci}$, it is also reported in the $-$ waste characterization report.

\subsubsection{Calculate ${ }^{239} \mathrm{Pu}$ FGE}

If the LLCE waste is TRU, as identified in Section 2.4 .4 , the ${ }^{239} \mathrm{Pu}$ fissile gram equivalent (FGE) is calculated for the LLCE waste package.

1) The Tankchar table is opened, inner joined to the Radreg and LLCENuc tables for radiochemical records, where the data field (Layer Number) is equal to 99 and the data field [PU-239 FGE Factor] is greater than zero. This forms the current record set.

2) For each record in the current record set, the procedure below is followed.

i) The gram equivalent $(\mathrm{g})$ is calculated by dividing the curie content $(\mathrm{Ci})$ by the specific activity $(\mathrm{Ci} / \mathrm{g})$.

ii) The ${ }^{239} \mathrm{Pu} F \mathrm{FE}(\mathrm{g})$ is calculated by multiplying the gram equivalent by the ${ }^{239} \mathrm{Pu}$ correction factor. The ${ }^{239} \mathrm{Pu} F \mathrm{FE}$ is summed with all previous ${ }^{239} \mathrm{Pu}$ FGEs.

3) The results of these calculations are reflected in the LLCE Waste Characterization Calculation window, providing immediate user feedback on the total ${ }^{239} \mathrm{Pu} F \mathrm{FE}$ of the. LLCE waste package. The total ${ }^{239} \mathrm{Pu} F G E$ is also reported in the waste characterization report.

\subsubsection{Calculate Alpha Curie Content}

If the LLCE waste is TRU as identified in Section 2.4 .4 , the alpha curie content is calculated for the LLCE waste package.

1) The Tankchar table is opened, inner joined to the LLCENuc table for radiochemical records, where the data field [Layer Number] is equal to 99 and the data field [Aipha Emitter] is equal to one. This forms the current record set.

2) The alpha curie content ( $\mathrm{Ci}$ ) is calculated by summing the curie contents ( $\mathrm{Ci}$ ) for all of the records in the current record set.

3) The result of this calculation is reflected in the LLCE Waste Characterization Calculation window, providing immediate user feedback on the total alpha curie content of the LLCE waste package. The total alpha curie content is also reported in the waste characterization report.

\subsubsection{Calculate Heat Generation Rate for Waste Package}

The heat generation rate of the entire LLCE waste package is calculated as follows

1) The Tankchar table is opened, inner joined to the LLCENuc table for radiochemical records, where the data field [Layer Number] is equal to 99. This forms the current record set.

2) For each record in the current record set, the heat generation rate $(W)$ is calculated by multiplying the curie content $(\mathrm{Ci})$ by the heat generation (W/Ci). The total heat generation 
rate $(W)$ for the LLCE waste package is calculated by summing all the heat generation rates calculated in this step (2).

3) The total heat generation rate of the LLCE waste package calculated above is reported in the waste characterization report.

4) The internal volume of the LLCE waste container (ContVol $\mathrm{m}^{3}$ ) is converted to cubic feet (multiply by $35.31467 \mathrm{ft}^{3} / \mathrm{m}^{3}$ ). The total heat generation rate per cubic foot $\left(\mathrm{W} / \mathrm{ft}^{3}\right.$ ) of the LLCE waste package is calculated by dividing the total heat generation rate by the waste container volume $\left(\right.$ now $\mathrm{ft}^{3}$ ). This value, as well as a comparison to the current $0.1 \mathrm{~W} / \mathrm{ft}^{3}$ limit, is reported in the waste characterization report.

5) The comparison of the total heat generation rate per cubic foot of the LLCE waste package to the $0.1 \mathrm{~W} / \mathrm{ft}^{3}$ limit is reflected in the LLCE Waste Characterization Calculation window to provide immediate user feedback.

\subsubsection{Calculate Transportation Category}

The transportation categories Type $A_{1}$ Type $B$, or highway route controlled quantity of the LLCE waste package is then determined.

1) The Tankchar table is opened, inner joined to the Radreg table for radiochemical records, where the data field [Layer Number] is equal to 99 and the data field [DOT A2 Limit] is greater than zero. This forms the current record set.

2) For each record in the current record set, the curie content $(\mathrm{Ci})$ is divided by the U.S. Department of Transportation (DOT) $A_{2}$ limit (Ci) resulting in a DOT $A_{2}$ fraction. The DOT $A_{2}$ sum of fractions variable (SUM_A2) for the LLCE waste package is calculated by summing all the DOT $A_{2}$ fractions calculated in this step (2).

3) The sum of fractions variable (SUM_A2) calculated in step 2) determines the transportation category for the LLCE waste package as follows.

i) If SUM_A2 $\leq 1$, the LLCE waste package is Type A.

ii) If $1<$ SUM_A2 $\leq 3000$, the LLCE waste package is Type $B$.

iii) If SUM_A2 > 3000, the LLCE waste package is a highway route controlled quantity.

4) The results of these calculations are reflected in the LLCE Waste Characterization Calculation window to provide immediate user feedback on the transportation category of the LLCE waste package. The DOT $\mathrm{A}_{2}$ sum of fractions and the corresponding transportation category are also reported in the waste characterization report.

5) If the LLCE waste is identified as Type A, then step 10 in the LLCE Waste Characterization Calculation window, Calculate $\mathbf{D E}-\mathbf{C i}$, which is applicable only to greater-than-Type A waste, becomes inactive. The text in the inactive step will turn grey, and N/A will be displayed beside it. This provides immediate user feedback that this step is not required. 


\subsubsection{Calculate DE-Ci}

If the LLCE waste is greater than Type A, as identified in Section 2.4.9, the dose equivalent curie (DE-Ci) content for the LLCE waste package is calculated.

1) The Tankchar table is opened, inner joined to the Radreg table for radiochemical records, where the data field [Layer Number] is equal to 99 and the data field [DE-Ci Curie Factor] is greater than zero. This forms the current record set.

2) For each record in the current record set, the curie content $(\mathrm{Ci})$ is multiplied by the $\mathrm{DE}-\mathrm{Ci}$ factor, resulting in the $\mathrm{DE}-\mathrm{Ci}$ content $(\mathrm{Ci})$ for that record. The total $\mathrm{DE}-\mathrm{Ci}$ for the LLCE waste package is calculated by summing all the DE-Ci contents calculated in this step (2).

3) The result of this calculation is reflected in the LLCE Waste Characterization Calculation window, providing immediate user feedback on the total DE-Ci content of the LLCE waste package. The total DE-Ci content is also reported in the waste characterization report.

\subsubsection{Calculate for Hazardous Waste Codes}

Waste codes F001-F005 apply to all LLCE waste packages. Calculations are done to determine if other hazardous waste codes (D004-D011) apply to the LLCE waste package. These other waste codes apply if the concentration of the corresponding heavy metal contaminants in the LLCE waste matrix exceeds regulatory limits. Calculation of the application of D codes for the LLCE waste is based on 40 CFR 266, "Standards for the Management of Specific Hazardous Wastes and Specific Types of Hazardous Waste Management Facilities," Appendix VII, "Health-Based Limits for Exclusion of WasteDerived Residues," and WAC 173-303, "Dangerous Waste Regulations."

1) The Tankchar table is opened, inner joined to the Chemreg table for chemical records, where the data field [Layer Number] is equal to 99 . This forms the current record set.

2) For each record in the current record set, the content ( $\mathrm{g}$ ) is converted to milligrams (multiply by $1 \mathrm{E}+03 \mathrm{mg} / \mathrm{g}$ ). The content is then divided by the LLCE waste mass $(\mathrm{kg})$ to obtain the concentration $(\mathrm{mg} / \mathrm{kg}$ ). If this concentration is greater than 20 times the $\mathrm{D}$ code limit for the current chemical, the D code limit for that chemical applies to the LLCE waste package.

3) The results of these calculations are reflected in the LLCE Waste Characterization Calculation window, providing immediate user feedback on the applicable hazardous waste codes for the LLCE waste package. The F001-F005 waste codes, concentrations calculated above, and the applicable $\mathrm{D}$ codes are reported in the waste characterization report.

With the completion of Section 2.4.11, the LLCE Waste Characterization Process is complete. The Reports button in the LLCE Waste Characterization Calculation window now becomes active, and the user can view, print, or export the waste characterization report or status report for the LLCE. 


\subsection{CALCULATION EXAMPLES AND VERIFICATION}

The following sections show sample calculations verify the correctness of the calculations performed by LLCECALC. Each example in this section is taken from the synthetic single layer case of Section 8.1 of the user's manual. These examples can be reproduced using the files c1_1layer.edf and c1_1layer.av2 in the verification directory. In processing the files, accept all the defaults in LLCECALC to replicate the examples in this section.

\subsection{SELECTED CALCULATION EXAMPLES FROM THE ANALYZE GAMMA ASSAY DATA FILE PROCESS}

The analyze gamma assay data file process involves several numerical calculations. This section contains example calculations for the more involved of these numerical calculations to verify that LLCECALC calculates the correct numerical value according to the algorithms specified in Section 2.3 . It is also recommended that the user refer to Section 2.3 while reading this section.

\subsubsection{Activity Reduction for Change in Record Length}

LLCECALC is sometimes required to change the length of a record. When this happens, the activity reported for that record must be adjusted proportionally to the change in the length of the record. Section 2.3.4, step 3, part $v$, discusses the methodology used.

The following tables are appropriate portions of the Positions and Gammadata tables showing the positions and activities before and after the activity reduction and record length change of record 1000090 . Note that the position in the Gammadata table corresponds to the end position of the record in the Positions table. This record was reduced because the crane had moved in the reverse direction, and the point where the crane again began moving forward fell in the middle of record 1000090 .

From Positions table before:

\begin{tabular}{|c|c|c|}
\hline ( $1 \%$ & $53 / 12$ & 30 \\
\hline 1000090 & 8 & 9 \\
\hline
\end{tabular}

From Gammadata table before:

\begin{tabular}{|c|c|c|c|}
\hline r & \%os & 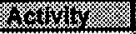 & Dertoris \\
\hline 1000090 & 9 & $1.70 \mathrm{E}-06$ & 1 \\
\hline
\end{tabular}

From Positions table after:

\begin{tabular}{|c|c|c|}
\hline 8 & 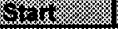 & (c) \\
\hline 1000090 & 8 & 8.3 \\
\hline
\end{tabular}

From Gammadata table after:

\begin{tabular}{|c|c|c|c|}
\hline 8. & $\gamma^{2}$ & $80 \times 146$ & 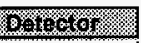 \\
\hline 1000090 & 8.3 & $5.10 \mathrm{E}-07$ & ب \\
\hline
\end{tabular}


The equation for activity reduction as specified in 2.3 .4 is:

$$
\text { NewActivity }=\frac{(\text { NewEnd }- \text { OrigStart })}{\text { (OrigEnd }- \text { OrigStart })} \times(\text { OldActivity })
$$

Substituting numbers from the above tables yields:

$$
\text { NewActivity }=\frac{(8.3 \mathrm{ft}-8.0 \mathrm{ft})}{(9.0 \mathrm{ft}-8.0 \mathrm{ft})} \times(1.70 \mathrm{E}-06 \mathrm{Ci})=5.10 \mathrm{E}-07 \mathrm{Ci}
$$

The status log output shows the following:

8/6/98 - 12:21 PM: Record ID 1000150 is inserted in the middle of record ID 1000090

8/6/98 - 12:21 PM: Record 1000090 new end position is now 8.30000 . Activity in record 101000090 has been reduced by $70.00000 \%$

The reduced activity calculated by LLCECALC and reported in the [Activity] data field in the Gammadata table is the same as that calculated in the equation above, from Section 2.3.4. It is also $70 \%$ less than the original activity as reported in the status log. The LLCECALC calculation of reduced activities is thus verified.

\subsubsection{Missing Activity Estimation}

LLCECALC estimates the occurrence of a missing activity by linear interpolation using the midpoints of the adjacent (previous and next) reported activities. The window shown below displays one such case where the value displayed (7.97320E-07) is the LLCECALC estimation of the activity.

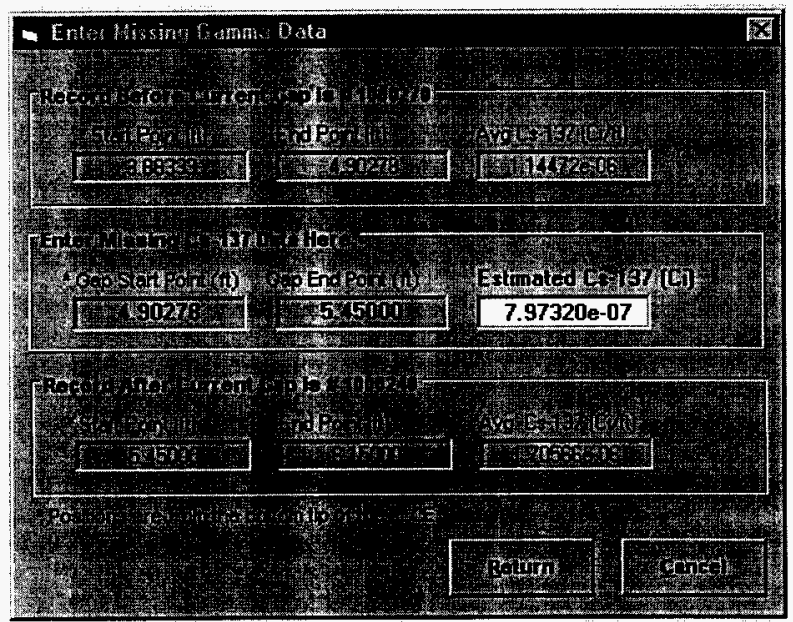


The following calculations verify the correctness of the data in this Enter Missing Gamma Data window. The midpoints are as follows:

$$
\begin{array}{ll}
\text { Midpoint of record \# 1000270: } & (4.90278+3.88333) / 2=4.39306 \\
\text { Midpoint of record \# 1000240: } & (6.15000+5.45000) / 2=5.8 \\
\text { Midpoint of the missing record: } & (5.45000+4.90278) / 2=5.17639 .
\end{array}
$$

Linear Interpolation using these midpoints yields an activity of 7.97316E-07 Ci as calculated below.

$$
\left[\left(\frac{5.1764-4.3931}{5.8000-4.3931}\right) \times\left(1.7057 \times 10^{-6}-1.1447 \times 10^{-6}\right)+1.1447 \times 10^{-6}\right] \times(5.4500-4.9028)=7.97316 \times 10^{-7}
$$

NOTE: Numerical precision was truncated in displaying the equation above to facilitate formatting.

This calculated activity, $7.97316 \times 10^{-7} \mathrm{Ci}$, is practically the same as the LLCECALC estimated activity, $7.97320 \mathrm{e}-07$, and the very small difference, $0.0005 \%$ error, is likely because LLCECALC carries more digits than are displayed in the above window. The hand calculation uses only one more digit than is displayed in the equation above. The LLCECALC calculation of missing activities is thus verified.

\subsubsection{Weighted Averaged Activity From Three Detectors}

LLCECALC uses activities reported from up to three detectors to calculate an averaged value to be used in the characterization of the LLCE waste. The following table is an appropriate portion of the Positions table showing the reported activities and percent errors in the activities as well as LLCECALC calculated weighted average of record 1000160 :

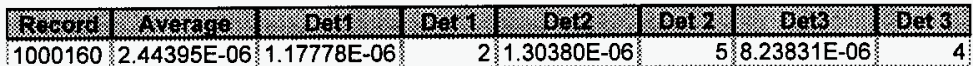

The equation for the weighted average as specified in Section 2.3 .9 is

$$
\text { Average }=\frac{\sum_{i=1}^{3} A_{c t} \times\left(1 / E \mathrm{Er}_{1}\right)^{2}}{\sum_{i=1}^{3}\left(1 / E \mathrm{Er}_{\mathrm{i}}\right)^{2}}
$$

The equation is the quotient of two sums. The following values are precalculated to simplify the form of the final equation. 
$\left(1 / \mathrm{Err}_{1}\right)^{2}=(1 / 2)^{2}=0.25$

Act $_{1} \times\left(1 / \text { Err }_{1}\right)^{2}=1.17778 \mathrm{E}-06 \times 0.25=2.94444 \mathrm{E}-07$

$\left(1 / \mathrm{Err}_{2}\right)^{2}=(1 / 5)^{2}=0.04$

$\mathrm{Act}_{2} \times\left(1 / \mathrm{Err}_{2}\right)^{2}=1.30380 \mathrm{E}-06 \times 0.04=5.21518 \mathrm{E}-08$

$\left(1 / \mathrm{Err}_{3}\right)^{2}=(1 / 4)^{2}=0.0625$

$\mathrm{Act}_{3} \times\left(1 / \mathrm{Err}_{3}\right)^{2}=8.23831 \mathrm{E}-06 \times 0.0625=5.14894 \mathrm{E}-07$

$\mathrm{Act}_{1} \times\left(1 / \mathrm{Err}_{1}\right)^{2}+\mathrm{Act}_{2} \times\left(1 / \mathrm{Err}_{2}\right)^{2}+\mathrm{Act}_{3} \times\left(1 / \mathrm{Err}_{3}\right)^{2}=$

$=2.94444 \mathrm{E}-07+5.21518 \mathrm{E}-08+5.14894 \mathrm{E}-07$

$=8.61491 \mathrm{E}-07$

$\left(1 / \mathrm{Err}_{1}\right)^{2}+\left(1 / \mathrm{Err}_{2}\right)^{2}+\left(1 / \mathrm{Err}_{3}\right)^{2}=0.25+0.04+0.0625=0.3525$

$$
\text { Average }=\frac{\sum_{i=1}^{3} A_{1} \times\left(1 / \mathrm{Err}_{1}\right)^{2}}{\sum_{i=1}^{3}\left(1 / E r_{1}\right)^{2}}=\frac{8.61491 E-07}{0.3525}=2.44395 E-06
$$

The hand calculation is complete. The weighted averaged activity calculated by LLCECALC and reported in the [Average] data field in the Positions table is the same as that calculated in the equation from Section 2.3.9. The LLCE calculation of the weighted average is thus verified.

\subsubsection{Calculation of the Shielding Correction Factors for Detectors 2 and 3}

LLCECALC calculates the shielding correction factors for detectors No. 2 and No. 3 based on the activities these two detectors report when detector No. 1 reports activities.

The algorithm used to calculate these shielding correction factors is specified in Section 2.3.6 and is demonstrated here using an Excel spreadsheet. The numbers used in the spreadsheet are taken from the Gammadata table immediately after step 5 of the Analyze Gamma Assay Data file process is complete. 


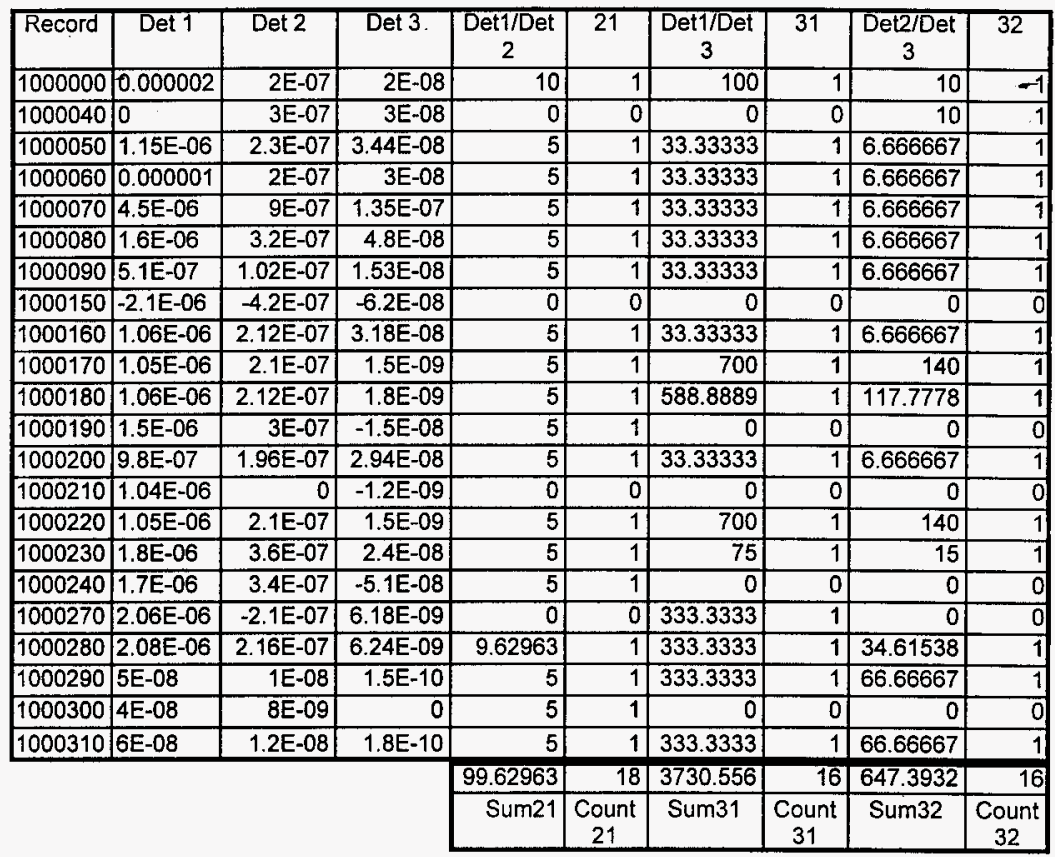

Shielding Correction Factor Detector $2 \quad 5.534979$

\begin{tabular}{ll|l|}
\hline Shielding Correction Factor Detector 3 & 233.1597 \\
\hline
\end{tabular}

The status log output shows the following:

8/6/98 - 07:05 PM: Detector 2 shielding correction factor estimated to be 5.53498

8/6/98 - 07:05 PM: Detector 3 shielding correction factor estimated to be 233.15972

The shielding correction factors for detectors No. 2 and No. 3 calculated by LLCECALC and reported in the status log output are the same as those calculated in the spreadsheet above. The LLCE calculation of the shielding correction factors is thus verified.

\subsection{CALCULATION EXAMPLES FROM THE LLCE WASTE CHARACTERIZATION PROCESS}

The LLCE Waste Characterization Process involves many numerical calculations within its algorithms. This section contains examples of the calculations required for one chemical constituent and one radionuclide constituent in the LLCE Waste Characterization Process. It is recommended that Section 2.4 be referred to while reading this section. 
For the synthetic single layer case of Section 8.1 of the users guide, there are three chemical constituents and six radionuclide constituents including ${ }^{137} \mathrm{Cs}$. The calculations necessary for the chemical mercury and the radionuclide ${ }^{241} \mathrm{Pu}$ will be documented.

There are several tables from the EDF that are accessed and examined during the LLCE Waste Characterization Process. The appropriate portions of these tables have been copied and printed on the next page for reference in the calculations of this section.

\subsubsection{Calculate Radionuclide and Chemical Content}

LLCE waste mass (LLCEMass) is $50 \mathrm{~kg}$, by accepting the default. The equation for calculating constituent content, as specified in Section 2.4 .1 .2 , is

$$
\text { LLCEContent }=\frac{(\text { TankContent }) \times(\text { CS_LLCE })}{\text { CS_Tank }}
$$
Layer table.

The TankContent and CS_Tank values are in the Tankchar table. The CS_LLCE value is in the

For the chemical mercury:

$$
\text { LLCEContent }=\frac{(500000 \mathrm{~g}) \times(4.68808 \mathrm{E}-05 \mathrm{Ci})}{100 \mathrm{Ci}}=2.34404 \mathrm{E}-01 \mathrm{~g}
$$

For the radionuclide ${ }^{241} \mathrm{Pu}$ :

$$
\text { LLCEContent }=\frac{(500000 \mathrm{Ci}) \times(4.68808 \mathrm{E}-05 \mathrm{Ci})}{100 \mathrm{Ci}}=2.34404 \mathrm{E}-01 \mathrm{Ci}
$$

The calculations and equations presented in this section are illustrative only. It is not possible to perform a direct comparison to data produced by LLCECALC to verify this case. The user is referred to Volume III of this document for a detailed discussion of the validation procedure, where multiple detailed examples are used to confirm the methodology used here.

\subsubsection{Check LLCE Waste Against SARP Limits}

SARP (McCormick 1997) limits are in the Radreg table. The SARP limit for ${ }^{241} \mathrm{Pu}$ is $0.179 \mathrm{Ci}$, and the ${ }^{241} \mathrm{Pu}$ content calculated in Section 3.2.1 is $0.2344 \mathrm{Ci}$, so the SARP limit is exceeded. There are no SARP limits for chemical constituents. This agrees with characterization of LLCECALC. 

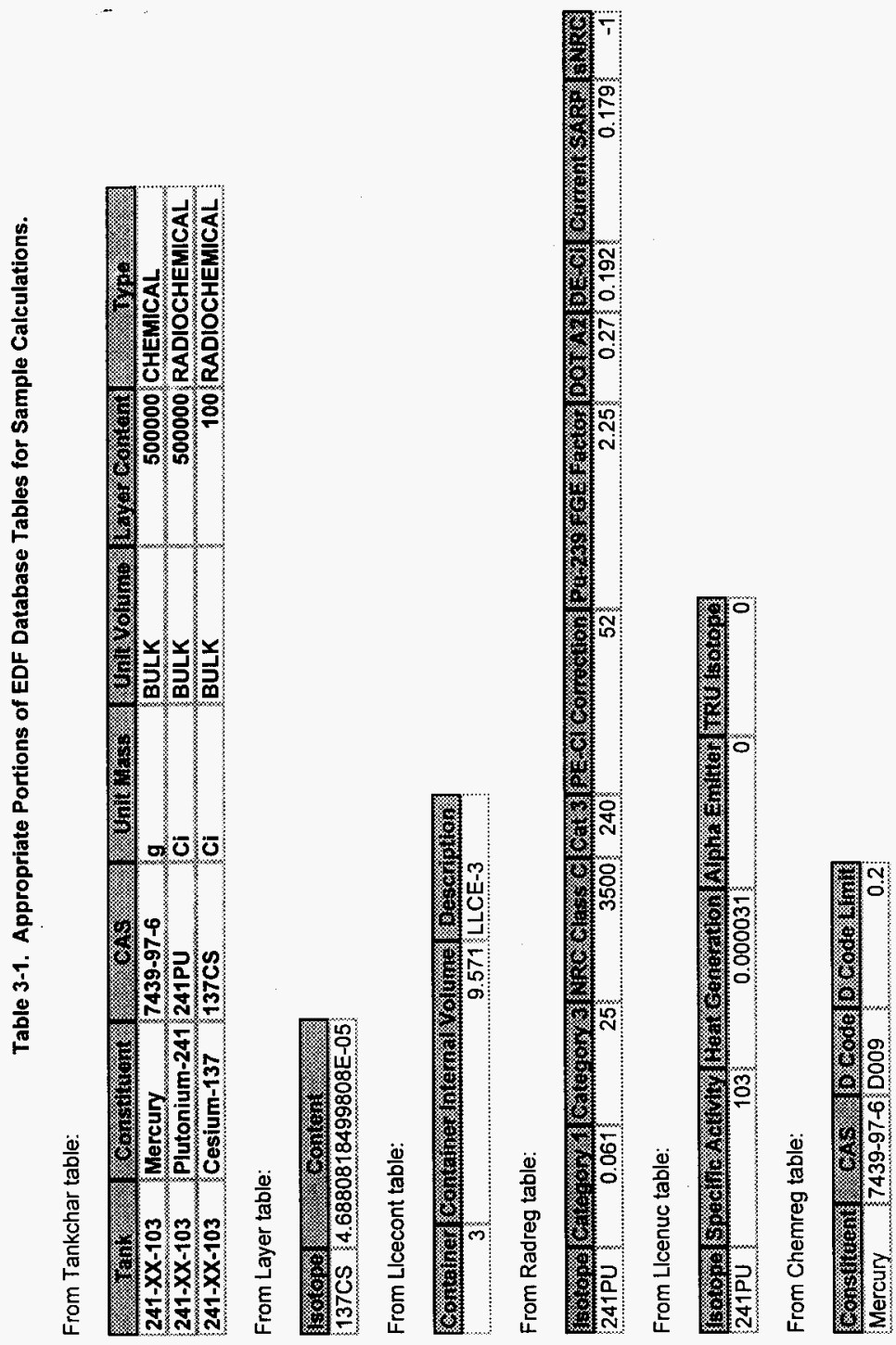


\subsubsection{Calculate for Category 1 and 3 LLW}

The LLCE waste is characterized by these limits using a sum-of-fractions method. A fraction must be calculated for each appropriate constituent for each of these limits. The fraction for Category 1 LLW, Category 3 LLW, and the ISB Cat 3 Noncombustion is calculated as follows:

$$
\text { Fraction }=\frac{\text { LLCEContent }}{(\text { ContVol }) \times(\text { Limit })}
$$

The ContVol value is in the Llcecont table; in this case, it is container 3 . The Limit values are in the Radreg table. Chemical constituents do not have these limits.

For ${ }^{241} \mathrm{Pu}$ Category 1 LLW:

$$
\text { Fraction }=\frac{2.34404 \mathrm{E}-01 \mathrm{Ci}}{\left(9.571 \mathrm{~m}^{3}\right) \times\left(0.061 \mathrm{Ci} / \mathrm{m}^{3}\right)}=4.01493 \mathrm{E}-01
$$

For ${ }^{241} \mathrm{Pu}$ Category 3 LLW:

$$
\text { Fraction }=\frac{2.34404 \mathrm{E}-01 \mathrm{Ci}}{\left(9.571 \mathrm{~m}^{3}\right) \times\left(25 \mathrm{Ci} / \mathrm{m}^{3}\right)}=9.79643 \mathrm{E}-04
$$

For ${ }^{241} \mathrm{Pu}$ ISB Cat 3 Noncombustion:

$$
\text { Fraction }=\frac{2.34404 \mathrm{E}-01 \mathrm{Ci}}{\left(9.571 \mathrm{~m}^{3}\right) \times\left(240 \mathrm{Ci} / \mathrm{m}^{3}\right)}=1.02046 \mathrm{E}-04
$$

The fraction for NRC class $C$ is calculated using one of two possible concentrations-one based on mass and one based on volume. These concentrations are calculated as follows:

$$
\begin{gathered}
\text { MassConc }=\frac{(1 \mathrm{E}+09 \mathrm{nCi} / \mathrm{Ci}) \times(\text { LLCEContent })}{(1 \mathrm{E}+03 \mathrm{~g} / \mathrm{kg}) \times(\text { LLCEMass })} \\
\text { VolConc }=\frac{(\text { LLCEContent }) \times(\text { LLCEDensity })}{\text { LLCEMass }}
\end{gathered}
$$

The LLCEDensity value is based on the density of steel and is $7840.45 \mathrm{~kg} / \mathrm{m}^{3}$.

$$
\begin{gathered}
\text { MassConc }=\frac{(1 \mathrm{E}+09 \mathrm{nCi} / \mathrm{Ci}) \times(2.34404 \mathrm{E}-01 \mathrm{Ci})}{(1 \mathrm{E}+03 \mathrm{~g} / \mathrm{kg}) \times(50 \mathrm{~kg})}=4.68808 \mathrm{E}+03 \mathrm{nCi} / \mathrm{g} \\
\text { VolConc }=\frac{(2.34404 \mathrm{E}-01 \mathrm{Ci}) \times\left(7840.45 \mathrm{~kg} / \mathrm{m}^{3}\right)}{50 \mathrm{~kg}}=3.67567 \mathrm{E}+01 \mathrm{Ci} / \mathrm{m}^{3}
\end{gathered}
$$


The fraction is calculated by dividing the concentration by the NRC class $\mathrm{C}$ limit. The appropriate concentration is used based on the units of the limit as specified by the data field [sNRC] in the Radreg table.

$$
\text { Fraction }=\frac{4.68808 \mathrm{E}+03 \mathrm{nCi} / \mathrm{g}}{3500 \mathrm{nCi} / \mathrm{g}}=1.33945
$$

To calculate the total fractions for the entire LLCE waste package, the fractions calculated above for ${ }^{241} \mathrm{Pu}$ for each limit are summed with the fractions calculated for all other appropriate constituents.

The calculations and equations presented in this section are illustrative only. It is not possible to perform a direct comparison to data produced by LLCECALC to verify this case. The user is referred to Volume III of this document for a detailed discussion of the validation procedure, where multiple detailed examples are used to confirm the methodology used here.

\subsubsection{Calculate for TRU Waste}

The LLCE waste is characterized by the $100 \mathrm{nCi} / \mathrm{g}$ TRU limit using a sum-of-fractions method. A fraction must be calculated then for each appropriate constituent for this limit. The fraction for TRU is calculated as follows.

$$
\text { TRU_Fraction }=\frac{(1 \mathrm{E}+09 \mathrm{nCi} / \mathrm{Ci}) \times(\text { LLCEContent })}{(1 \mathrm{E}+03 \mathrm{~g} / \mathrm{kg}) \times(\text { LLCEMass }) \times(\text { TRU_Limit })}
$$

The TRU_Limit value is the $100 \mathrm{nCi} / \mathrm{g}$ mentioned above. The total TRU fraction for the entire LLCE waste package is calculated by summing the TRU fractions for all of the appropriate constituents. Only those constituents identified as TRU isotopes in the Llcenuc table (data field [TRU isotope]) have their fractions calculated and included in the total sum. ${ }^{24} \mathrm{Pu}$ is not a TRU isotope, so this calculation is not done in this example.

The calculations and equations presented in this section are illustrative only. This particular verification case, when including all isotopes, characterizes as TRU. The user is referred to Volume III of this document for a detailed discussion of the validation procedure, where multiple detailed examples are used to confirm the methodology used here.

\subsubsection{Calculate PE-Ci}

The LLCE waste PE-Ci is characterized using a sum-of-fractions method. A fraction must be calculated then for each appropriate constituent. There are three fractional values reported in the PE-Ci section of the status or characterization report, and these fractional values are calculated as follows:

$$
\begin{gathered}
\text { CurieContent }=\text { LLCEContent } \\
\text { GramEquivalent }=\frac{\text { LLCEContent }}{\text { SpecificActivity }}
\end{gathered}
$$

$$
P E-C i \_ \text {Fraction }=\frac{(\text { LLCEContent }) \times\left(P E-C i \_ \text {Factor }\right)}{\text { SpecificActivity }}
$$



table.

The SpecificActivity value is in the LIcenuc table. The PE-Ci_Factor value is in the Radreg For ${ }^{241} \mathrm{Pu}$ :

$$
\begin{gathered}
\text { CurieContent }=2.34404 \mathrm{E}-01 \mathrm{Ci} \\
\text { GramEquivalent }=\frac{2.34404 \mathrm{E}-01 \mathrm{Ci}}{103 \mathrm{Ci} / \mathrm{g}}=2.27577 \mathrm{E}-03 \mathrm{~g} \\
\mathrm{PE}-\text { Ci_Fraction }=\frac{(2.34404 \mathrm{E}-01 \mathrm{Ci}) \times(52)}{103 \mathrm{Ci} / \mathrm{g}}=1.18340 \mathrm{E}-01 \mathrm{~g}
\end{gathered}
$$

To calculate the total curie content, total gram equivalent, and total PE-Ci fraction for the entire LLCE waste, the values/fractions calculated above are summed with the values/fractions calculated for all other appropriate constituents. Only those constituents with PE-Ci correction factors greater than zero in the Radreg table, in the [PE-Ci Correction Factor] data field, have the fractional values calculated and included in the total sums.

The calculations and equations presented in this section are illustrative only. It is not possible to perform a direct comparison to data produced by LLCECALC to verify this case. The user is referred to Volume III of this document for a detailed discussion of the validation procedure, where multiple detailed examples are used to confirm the methodology used here.

\subsubsection{Calculate ${ }^{239} \mathrm{Pu}$ FGE}

The LLCE waste ${ }^{239} \mathrm{Pu}$ FGE is calculated using a sum of fractions method. A fraction must be calculated then for each appropriate constituent. The fraction for ${ }^{239} \mathrm{Pu} F \mathrm{FEE}$ is calculated as follows.

$$
\text { FGE_Fraction }=\frac{(\text { LLCEContent }) \times(\text { FGE_Factor })}{\text { SpecificActivity }}
$$

The FGE-Factor value is in the Radreg table.

For ${ }^{241} \mathrm{Pu}$ :

$$
\text { FGE_Fraction }=\frac{(2.34404 \mathrm{E}-01 \mathrm{Ci}) \times(2.25)}{103 \mathrm{Ci} / \mathrm{g}}=5.12048 \mathrm{E}-03 \mathrm{~g}
$$

To calculate the total ${ }^{239}$ Pu FGE fraction for the entire LLCE waste, the fraction calculated above is summed with the fractions calculated for all other appropriate constituents. Only those constituents with ${ }^{239} \mathrm{Pu}$ FGE correction factors greater than zero in the Radreg table, data field [Pu-239 FGE Factor], have the fractions calculated and included in the total sum. 
The calculations and equations presented in this section are illustrative only. It is not possible to perform a direct comparison to data produced by LLCECALC to verify this case. The user is referred to Volume III of this document for a detailed discussion of the validation procedure, where multiple detailed examples are used to confirm the methodology used here.

\subsubsection{Calculate Alpha Curie Content}

The LLCE waste alpha curie content is calculated by summing the curie content (LLCEContent) of all appropriate constituents. Only those constituents identified as alpha emitters in the Licenuc table (data field [Alpha Emitter]) have the curie contents included in the total sum. ${ }^{24} \mathrm{Pu}$ is not an alpha emitter, so its curie content is not added to the total sum in this example.

It is not possible to perform a direct comparison to data produced by LLCECALC to verify this case. The user is referred to Volume III of this document for a detailed discussion of the validation procedure, where multiple detailed examples are used to confirm the methodology used here.

\subsubsection{Calculate Heat Generation Rate for Waste Package}

The LLCE waste heat generation rate is calculated by summing the heat generation rate of each appropriate constituent. The heat generation rate for a constituent is calculated as follows:

$$
\text { Heat_Rate }=(\text { LLCEContent }) \times(\text { HeatGeneration })
$$

The HeatGeneration value is in the Llcenuc table.

For ${ }^{241} \mathrm{Pu}$ :

$$
\begin{aligned}
\text { Heat_Rate } & =(2.34404 \mathrm{E}-01 \mathrm{Ci}) \times(3.10 \mathrm{E}-05 \mathrm{~W} / \mathrm{Ci}) \\
& =7.26653 \mathrm{E}-06 \mathrm{~W}
\end{aligned}
$$

To calculate the heat generation rate for the entire LLCE waste, the heat generation rate calculated above is summed with the heat generation rates calculated for all other appropriate constituents. Chemical constituents do not have heat generation rates.

The calculations and equations presented in this section are illustrative only. It is not possible to perform a direct comparison to data produced by LLCECALC to verify this case. The user is referred to Volume III of this document for a detailed discussion of the validation procedure, where multiple detailed examples are used to confirm the methodology used here.

\subsubsection{Calculate Transportation Category}

The LLCE waste transportation category is characterized using a sum-of-fractions method. A fraction must be calculated for each appropriate constituent. The fraction for the transportation category is calculated as follows:

$$
\text { DOT_Fraction }=\frac{\text { LLCEContent }}{\text { DOT_Limit }}
$$

The DOT_Limit value is in the Radreg table. 
For ${ }^{241} \mathrm{Pu}$ :

$$
\text { DOT_Fraction }=\frac{2.34404 \mathrm{E}-01 \mathrm{Ci}}{0.27 \mathrm{Ci}}=8.68163 \mathrm{E}-01
$$

To calculate the total DOT fraction for the entire LLCE waste, the fraction calculated above is summed with the fractions calculated for all other appropriate constituents. Only those constituents with DOT $A_{2}$ limits greater than zero in the Radreg table, data field [DOT A2 Limit], have the DOT fractions calculated and included in the total sum.

The calculations and equations presented in this section are illustrative only. It is not possible to perform a direct comparison to data produced by LLCECALC to verify this case. The user is referred to Volume III of this document for a detailed discussion of the validation procedure, where multiple detailed examples are used to confirm the methodology used here.

\subsubsection{Calculate DE-Ci}

The LLCE waste DE-Ci is calculated by summing the DE-Ci for each appropriate constituent. The DE-Ci for a constituent is calculated as follows:

$$
\mathrm{DE}-\mathrm{C} \mathbf{i}=(\text { LLCEContent }) \times(\mathrm{DE}-\mathrm{Ci} \text { _Factor })
$$

The DE-Ci_Factor value is in the Radreg table.

For ${ }^{241} \mathrm{Pu}$ :

$$
\mathrm{DE}-\mathrm{C} \mathbf{i}=(2.33404 \mathrm{E}-01 \mathrm{Ci}) \times(0.192)=4.50056 \mathrm{Ci}
$$

To calculate the DE-Ci for the entire LLCE waste, the DE-Ci calculated above is summed with the DE-Ci calculated for all other appropriate constituents. Only those constituents with DE-Ci factors greater than zero in the Radreg table, data field [DE-Ci Curie Factor], have the DE-Ci values calculated and included in the total sum.

The calculations and equations presented in this section are illustrative only. It is not possible to perform a direct comparison to data produced by LLCECALC to verify this case. The user is referred to Volume III of this document for a detailed discussion of the validation procedure, where multiple detailed examples are used to confirm the methodology used here.

\subsubsection{Calculate for Hazardous Waste Codes}

The LLCE waste hazardous waste codes characterization is done by comparing the appropriate constituent concentration to the corresponding limit. The concentration for a constituent is calculated as follows:

$$
\text { HazardConc }=\frac{(1 \mathrm{E} 03 \mathrm{mg} / \mathrm{g}) \times(\text { LLCEContent })}{\text { LLCEMass }}
$$

For mercury:

$$
\text { HazardConc }=\frac{(1 \mathrm{E} 03 \mathrm{mg} / \mathrm{g}) \times(2.34404 \mathrm{E}-01 \mathrm{~g})}{50 \mathrm{~kg}}=4.68808 \mathrm{mg} / \mathrm{kg}
$$


The concentration calculated above is compared to 20 times the appropriate $\mathrm{D}$ code limit as specified in the Chemreg table, data field [D Code Limit]. In this case, the concentration of $4.688 \mathrm{mg} / \mathrm{kg}$ is larger than $20^{*}(0.2 \mathrm{mg} / \mathrm{kg})=4 \mathrm{mg} / \mathrm{kg}$, so the D009 Code Limit for Mercury is exceeded. This agrees with the characferization report from LLCECALC.

\subsubsection{Summary of LLCE Waste Characterization Calculations}

An example of each of the 11 steps of the characterization process have been demonstrated above. The following LLCE Waste Characterization window is displayed at the conclusion of the characterization for this test case file, c1_1layer.edf and c1_1layer.av2:

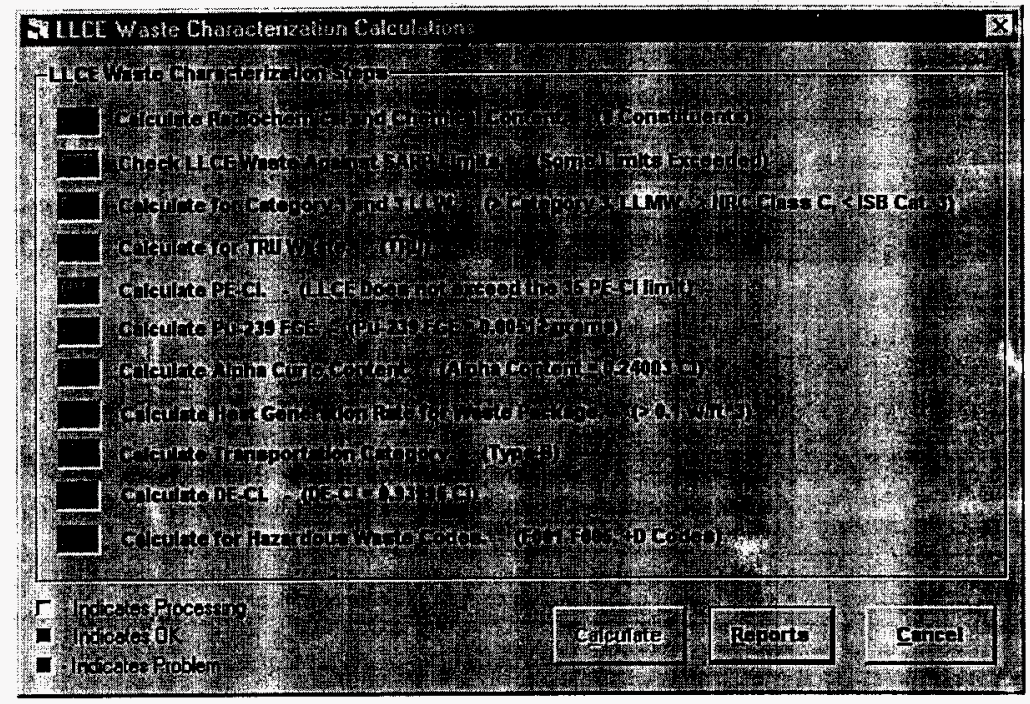

The user should refer to Volume III of this document for a detailed discussion and confirmation for this test case using all nine constituents. The Volume III verifies that LLCECALC correctly performs all the calculations required for characterization.

\subsection{EDF AND MAIN DATABASE FILE FORMAT}

\subsection{ORIGINATING DATABASE FILE: LLCEINFO.BIN}

LLCEDATA makes use of the database file /ceinfo.bin to create the EDF. The /ceinfo.bin file is a compressed and encrypted MS Access ${ }^{4}$ database. When the LLCEDATA program is executed, it automatically uncompresses the $\| c e$ info. bin file and renames it $/$ ceinfo mab. This uncompressed file,

${ }^{4}$ MS Access is a trademark of Microsoft Corporation. 
IIceinfo mdb contains all the information needed by LLCEDATA to allow the user to make the necessary selections to create an EDF. Appendices A1 and A2 contain a detailed description of this file and the format of the tables it contains. Table 4-1 shows each database table name and by a short description of the table.

Table 4-1. Description of //ceinfo.bin (//ceinfo.mdb) Database Tables.

\begin{tabular}{|l||l|}
\hline \hline Database Table Name & \\
\hline \hline Chemreg & $\begin{array}{l}\text { Contains a list of all chemical regulatory limits. The limits are the D Code } \\
\text { Limits for dangerous waste identification. }\end{array}$ \\
\hline LLCECont & Contains physical data for all LLCE waste containers, such as size, weight. \\
\hline LLCEGeom & Contains all known geometry correction factors for LLCE. \\
\hline LLCENuc & $\begin{array}{l}\text { Contains physical data for all radionuclides, including specific activity, half } \\
\text { life, heat generation, and transuranic and alpha-emitter identification. }\end{array}$ \\
\hline LLCEPhys & $\begin{array}{l}\text { Contains the physical data for all known LLCE, including a description, size, } \\
\text { weight, drawing data, and originating tank farm. }\end{array}$ \\
\hline LLCEGamma & Contains the list of all valid measured gamma isotopes, currently just ${ }^{137}$ Cs. \\
\hline Radreg & $\begin{array}{l}\text { Contains the list of all radionuclide regulatory limits. These limits include } \\
\text { Cat 1, Cat 3, NRC Class C, ISB noncombustion, PE-Ci factors, } \\
\text { factors, Pu FGE } \\
\text { the identification of sNRC for proper unit checking. These limits are } \\
\text { identified per isotope. }\end{array}$ \\
\hline Tankchar & $\begin{array}{l}\text { Contains the list of all tank waste characterization data. Per tank, this } \\
\text { information includes the constituent, layer content, layer number, and source } \\
\text { of the data (information oniy). }\end{array}$ \\
\hline Element & $\begin{array}{l}\text { Contains the list of all radionuclides and chemicals. Includes description, } \\
\text { CAS, name, and chemical formula (just for chemicals). }\end{array}$ \\
\hline
\end{tabular}

McCormick. W. A., 1997, Safely Analysis Report for Packaging (Onsite) Long-Length Contaminated Equipment Transport System, HNF-SD-TP-SARP-013, Rev. 0, prepared by Waste Management Federal Services, Inc., Northwest Operations for Fluor Daniel Hanford, Inc., Richland, Washington.

CAS $=$ Chemical Abstracts Service.

$\mathrm{DE}-\mathrm{C} \mathrm{i}=$ Dose equivalent curie.

DOT = U.S. Department of Transportation.

FGE $=$ Fissile gram equivalent.

ISB = Interim safety basis.

LLCE $=$ Long-Length Contaminated Equipment

NRC $=$ U.S. Nuclear Regulatory Commission

$\mathrm{PE}-\mathrm{C} \mathrm{i}=$ Plutonium equivalent curie

SARP = Safety analysis report for packaging.

LLCEDATA utilizes the contents of this database when the user is creating an EDF. The user is not allowed access to modify the contents of the llceinfo file. The code custodian is the only authorized person to make permanent changes to this file. This policy will allow the main database to be consistent, uniform, and accurate among all users. The table that is most likely to need periodic updating, yet being of critical importance to the use of LLCEDATA, is the Tankchar table.

While it is not possible for the user to make modifications to this //ceinfo file, it may be necessary to make modifications to the contents of any of these tables for the specific LLCE activity in use. An example where the user may need to do this would be to correct LLCE specifics, such as weight, which may be missing in the table. The user is allowed to make modifications to the use of any data stored in 
these tables; i.e., when data is extracted from the Ilceinfo tables by LLCEDATA, the user is allowed to edit any table entry as it is being used to create the EDF. However, the change is only stored in the current EDF. The main liceinfo file remains unmodified whenever the user selects any "edit data" function in LLCEDAT $\bar{A}$. LLCEDATA keeps a running status of all changes made to the use of the contents of the /lceinfo fle that are stored in the EDF. This running status is maintained in the status report and can serve as a historical document to indicate exactly what modifications took place to the LLCE analysis that deviates from the established IIceinfo database file.

\subsection{THE EDF}

The EDF is an MS Access file that is created by LLCEDATA in the first phase of an LLCE analysis. After the first phase of the analysis, the EDF contains three major items of user-supplied or -selected information: (1) the identification and physical description of the LLCE, (2) the identification of the waste tank and content, and (3) the determination of the geometry factors. During LLCEDATA's Finish Data File (step four), LLCEDATA adds the SARP-approved (McCormick 1997) container data, the nuclide constants and limits, the chemical limits, and the valid gamma list to the EDF. The EDF is now ready for LLCECALC.

LLCECALC read the LLCEDATA-generated EDF and the GEA-produced AV2 file and characterizes the LLCE. When the LLCECALC program terminates, the EDF file contains all the information for the LLCE; i.e., LLCECALC adds the characterization information to the EDF that was originally created by LLCEDATA. The EDF is then a single file maintaining a complete history of the LLCE, including analysis methodology, assumptions, and characterization that can be used to archive the LLCE activity. This archival history is maintained by unitizing the tables shown in Table 4-1, combined with any user-generated modifications or additions to these tables and the characterization information from LLCECALC. Appendices A1 - A4 contain the details of the format of the EDF database. Table 4-2 shows each database table name and a brief description of the table.

The user has access to the contents of the EDF by using either LLCEDATA or LLCECALC to print reports. Volume I, the user's manual, has detailed information on how the user can print these reports. 
Table 4-2. Equipment Data File (EDF) Database Tables.

\begin{tabular}{|c|c|}
\hline $\begin{array}{l}\text { Database } \\
\text { Table Name }\end{array}$ & Description \\
\hline User & $\begin{array}{l}\text { Contains user data for the EDF. It has general information about the program user, date, LLCE } \\
\text { descriptive data, waste tank, units, and status of what has been completed and edited in the } \\
\text { file. }\end{array}$ \\
\hline Chemreg & $\begin{array}{l}\text { Contains a tist of all chemical regulatory limits for the selected LLCE. This list is a copy from } \\
\text { the I/ceinfo.mdb file. }\end{array}$ \\
\hline LLCECont & $\begin{array}{l}\text { Contains physical data for the waste container that will be used for the selected LLCE. This list } \\
\text { is a copy from the Ilceinfo.mdb file. }\end{array}$ \\
\hline LLCEGeom & $\begin{array}{l}\text { Contains geometry correction factors for the selected LLCE. If available, these factors came } \\
\text { from Ilceinfo.mdb; otherwise, they were entered by the user. }\end{array}$ \\
\hline LLCENuC & $\begin{array}{l}\text { Contains a copy of the LLCENuc table extracted from the /lceinfo.mdb database file. This table } \\
\text { contains physical data for all radionuclides, including specific activity, half life, heat generation, } \\
\text { and transuranic and alpha-emitter identification. }\end{array}$ \\
\hline LLCEPhys & $\begin{array}{l}\text { Contains the physical data for the selected LLCE. The specific, single LLCE information in this } \\
\text { table is extracted from Ilceinfo.mdb, supplemented by any user supplied data. }\end{array}$ \\
\hline LLCEGamma & Contains a copy of the LLCEGamma table extracted from the /ceinfo.mdb database file. \\
\hline Radreg & Contains a copy of the Radreg table extracted from the liceinfo.mdb database file. \\
\hline Tankchar & $\begin{array}{l}\text { Contains the tank waste characterization data for the selected waste tank. The specific, single } \\
\text { waste tank information in this table is extracted from /lceinfo.mdb, supplemented by any } \\
\text { user-supplied data. }\end{array}$ \\
\hline Status & $\begin{array}{l}\text { Contains the running status of all significant events that take place while running the } \\
\text { LLCEDATA and LLCECALC programs. This table records all changes (edits to the contents of } \\
\text { the Ilceinfo.mdb file) made by the user and records all the selections made by the user or by the } \\
\text { program. This table is used to generate the Status report from either LLCEDATA or } \\
\text { LLCECALC. }\end{array}$ \\
\hline Gammadata & $\begin{array}{l}\text { Initially contains a copy of the Rawdata table data. After the AV2 file is read by LLCECALC, } \\
\text { which occurs when the user selects Process Gamma in the Analyze Gamma Assay Data } \\
\text { window, the total LLCE content for each of the constituents will be found in this table. The } \\
\text { activity is listed by detector number and percent error. }\end{array}$ \\
\hline Rawdata & $\begin{array}{l}\text { Contains the gamma assay information as read from the AV2 file. This is the uncorrected raw } \\
\text { data. }\end{array}$ \\
\hline Positions & $\begin{array}{l}\text { Generated by LLCECALC. Contains }{ }^{137} \mathrm{Cs} \text { data extracted from the gamma assay records. } \\
\text { Includes }{ }^{137} \mathrm{Cs} \text { inventory and percent error from all three detectors, the crane position for each } \\
\text { record, and the waste tank layer containing the particular part of the LLCE. }\end{array}$ \\
\hline Layer & $\begin{array}{l}\text { Contains the LLCECALC estimate of }{ }^{137} \mathrm{Cs} \text { content for every tank waste layer defined. The sum } \\
\text { of the }{ }^{137} \mathrm{Cs} \text { content for all layers is equal to the total }{ }^{137} \mathrm{Cs} \text { content for the entire LLCE. }\end{array}$ \\
\hline
\end{tabular}

AV2 = Gamma assay file.

$E D F=$ Equipment data file

LLCE $=$ Long-Length Contaminated Equipment. 


\subsection{SUMMARY AND RECOMMENDATIONS}

The two programs, LLCEDATA and LLCECALC, have been written to characterize the contamination that remains on an LLCE after it has been washed by water jets during retrieval from the high-level waste tanks at Hanford. This report has presented the technical basis and methodology of the characterization and has described the role of each program in the process. The limitations of the contamination characterization have been presented. These limitations are not associated with the software, but rather are due to assumptions and constraints imposed by the measurement process. The software has been extensively tested and has been shown to faithfully apply the characterization methodology. The testing and validation process is presented in the companion document, LLCEDATA and LLCECALC For Windows Version 1.0 Volume III: Verification and Validation.

As the measurement and analysis methods for LLCE characterization have been examined during the course of this software development, verification, and validation project, several recommendations or suggestions for improvements have surfaced as follows:

1) Database Administrator. The primary database file, /lceinfo.bin, contained in the program LLCEDATA will need to be periodically updated to reflect new knowledge of tank waste contents. When multiple waste layer data become available, the database will have to be extensively upgraded for each such tank. Also, as new equipment is planned for retrieval, dimensions, masses, geometry correction factors, and other data will have to be entered in the database. Because of this continuing need to modify the database and because of its importance to the LLCE characterization process, it is recommended that a person expert in these data be assigned to maintain the database and that only this person be authorized to make permanent changes to the database.

2) Verification of LLCE Radionuclide Ratios. A critical and as yet unverified assumption of the characterization process is that all radionuclides that contaminate the LLCE are in the same ratio to ${ }^{137} \mathrm{Cs}$ as the radionuclides of the tank waste. In particular, the TRU characterization of the LL.CE depends on this assumption. It was recommended in the original planning document for this system (Roach 1995) that the TRU characterization of the LLCE using tank waste radionuclide ratios be verified with neutron measurements performed on the retrieved LLCE. This has not been done. It is recommended that these measurements be done and, further, that one or two of the LLCE that have been retrieved be sampled, the contaminants ${ }^{137} \mathrm{Cs}$ and ${ }^{90} \mathrm{Sr}{ }^{90} \mathrm{Y}$ be analyzed, and the ratio be compared to that for the tank waste. This would help validate the use of tank waste ratios in the LLCE characterization, at least for the sampled equipment. The recommended neutron measurements are needed to help justify the "suspect TRU" trigger of $85 \mathrm{nCi} / \mathrm{g}$ (or $90 \mathrm{nCi} / \mathrm{g}$ ) that is now used in LLCECALC.

3) Multi-Layer Tank Waste Data. In its present form, the tank waste database does not include multiple layers. Therefore, when an LLCE is characterized for contamination, the analyzed ${ }^{137} \mathrm{Cs}$ content along its length is multiplied by the same ratios for other radionuclides, regardless of position along the LLCE. As discussed earlier in Section 1.1.4, actual tank waste occurs in layers. The neglect of layering and the consequent changes in radionuclide ratios relative to ${ }^{137} \mathrm{Cs}$ with position along the LLCE can introduce large uncertainties in the total computed LLCE activity. It is recommended that an effort be made to implement multi-layer models for tanks for which equipment retrieval is planned and that these models include at least two layers, one for the liquid-saturated sludge at the base of the tank and another for the partially saturated (partially dry) waste zone above. Further, an efficient method (rather than the "manual" procedure described in Volume I, the user's manual) should be devised for entering additional tank waste layers to the database.

4) Validation of GEA Software. The software developed at EG\&G Idaho (Killian and Hartwell 1988, Kiltian and Femec 1992, Killian and Sorensen 1992) for processing the 
measured gamma-ray spectra and producing peak intensities and \% errors should be validated by comparison to those obtained by independent methods or from commercially available spectra analysis programs. There are instances from the processed spectra that appear in the AV2 files where activities are reported as negative (not physically reasonable), but yet the \% error is not greater than 100 , implying that the result is valid. This behavior at low activities points out the need for independent validation of the processed peak intensities and \% error values.

5) New and Improved Gamma Detector Efficiency Calibration. A calibration procedure based on the use of point ${ }^{137} \mathrm{Cs}$ sources, one calibrated and of reasonably low activity and another of much higher activity, but not necessarily well-calibrated, should be considered. Point source measurements are made along the axis of the FRRDS where the LLCE would be located during retrieval. The point source measurements would be made for positions above and below the plane of the detectors so that the "line source response" can be constructed for all three detectors. For an LLCE with negligible self-absorption, this calibration procedure will not only produce valid efficiency factors for all three detectors but also will eliminate the need for the geometry and shielding correction factors used by LLCECALC. This improvement is significant because detectors No. 2 and No. 3 would now make measurements at each position that does not depend on detector No. 1; hence, the azimuthal dependence of activity at each position is obtained with the three detector results. In addition, the uncertainty associated with the method of obtaining detector No. 2 and No. 3 efficiency relative to detector No. 1 using data along the length of the LLCE is removed. Further, uncertainties introduced by the use of geometry and shielding correction factors derived from computer simulations are removed.

6) AV2 File Tank/Riser/LLCE Identifier. To positively ensure proper association of tank waste and equipment data with the measured gamma data, it would be better if the software used to acquire and process gamma spectra, creating the AV2 file, required the operator to enter the waste tank, riser number, and equipment identification before the LLCE retrieval is begun. Because this software is not part of the present development of LLCEDATA and LLCECALC, it was not possible to make such a modification as part of the current project.

There are two additional recommendations for improvements that are less significant than the others, but still worth considering, as follows.

1) It has been suggested (Kessler 1995) that the load cell in the crane that retrieves the LLCE be calibrated so that the actual mass of the LLCE is recorded. This then provides a more accurate figure for computing the contaminant concentration per unit mass than is obtained by simply using the "clean" mass of the LLCE recorded in the equipment database (EDF). For certain equipment, such as pumps, it may also provide information that may help to infer contaminant source location (interior or exterior to pump housing) and, therefore, may lead to better definition of the computer models needed for geometry correction factor simulations.

2) A second recommendation arises from a desire to simplify a very complicated process that LLCECALC must perform in order to assign gamma data records to position on the LLCE. The complication arises from the fact that gamma data for each detector are taken for 60 seconds without regard to crane position. In addition, the three detectors sometimes start and stop at slightly different times, complicating the process of assigning a single 1-ft travel interval to the three detector gamma records. If the gamma spectrometer start and stop times were slaved to the crane position encoder so that each detector counted gammas for the same $1 \mathrm{ft}$ of travel, then much of the complicated logic required to process the gamma data and relate it to LLCE position would be eliminated.

Future LLCE retrievals will likely be pumps and other items for which the assumptions used in computing the present geometry factors are no longer valid. The user must be certain that new values for 
the geometry factors are obtained for such equipment. In addition, contamination characterization results obtained for such equipment may have added uncertainty because of self-absorption effects.

\subsection{REFERENCES}

10 CFR 61, "Licensing Requirements for Land Disposal of Radioactive Waste," Code of Federal Regulations, as amended.

40 CFR 266, "Standards for the Management of Specific Hazardous Wastes and Specific Types of Hazardous Waste Management Facilities," Code of Federal Regulations, as amended.

WAC 173-303, "Dangerous Waste Regulations," Washington Administrative Code, as amended.

Ellefson, M. D., 1998, Hanford Site Solid Waste Acceptance Criteria, HNF-EP-0063-5, prepared by Waste Management Federal Services of Hanford, Inc., for Fluor Daniel Hanford, Inc., Richland, Washington.

Gedeon, S. R., 1996, Radionuclide Inventory of the T101AZ Thermocouple Tree From Riser 16A, WHC-SD-W151-CN-001, Rev. OC (thermocouple trees from other risers are included as appendices).

HNF-3169, 1998, LLCEDATA and LLCECALC for Windows Version 1.0, Volume I: User's Manual, Rev. 0, prepared by Waste Management Federal Services, Inc., Northwest Operations for Fluor Daniel Hanford, Inc., Richland, Washington.

HNF-3169, 1998, LLCEDATA and LLCECALC for Windows Version 1.0, Volume 1II: Software Verification and Validation, Rev. 0, prepared by Waste Management Federal Services, Inc., Northwest Operations for Fluor Daniel Hanford, Inc., Richland, Washington.

Kessler, S. F., 1996, Radioisotope Inventory of the T101AZ Thermocouple Tree from Riser 13D, WHC-SD-W151-CN-001, Rev. 0, Westinghouse Hanford Company, Richland Washington

Kessler, S. F., 1995, Radioisotope Inventory of the T101-AZ Thermocouple Tree Shipping Container (internal memo 8M730-SFK-95-010 to M. D. Ellefson, D. C. Hetzer, and E. M. Nordquist, December 11), Westinghouse Hanford Company, Richland, Washington.

Killian, E. W., and D. A. Femec, 1992, An Operator's Guide for VAXGAP: A Gamma-ray Spectral Analysis Package, EGG-2672, Edgerton, Germeshausen \& Grier, Idaho Falls, Idaho.

Killian, E. W., and T. C. Sorensen, 1992, "Concurrent Determination of Ambient Background Contribution to Low-Activity High-Resolution Gamma-Ray Spectra," Radioactivity \& Radiochemistry, Vol. 3 No. 1.

Killian, E. W., and J. K. Hartwell, 1988, VAXGAP: A Code for the Routine Analysis of Gamma-Ray Pulse-Height Spectra on a VAX Computer, EGG-2633, Edgerton, Germeshausen \& Grier, Idaho Falls, Idaho.

McCormick, W. A., 1997, Safety Analysis Report for Packaging (Onsite) Long-Length Contaminated Equipment Transport System, HNF-SD-TP-SARP-013, Rev. 0, prepared by Waste Management Federal Services, Inc., Northwest Operations for Fluor Daniel Hanford, Inc., Richland Washington. 
Nelson, J. V., 1998, Subject: Requirement for new geometry correction factors that take self-absorption of equipment into account (personal communication to R. D. Wilson, Waste Management Federal Services, Inc., Northwest Operations, April 9), Fluor Daniel Northwest, Inc., Richland, Washington.

Roach, H. L., 1995, Disposal of Tank Farm Long-Length Contaminated Equipment: Radiological and Chemical Characterization Plan, WHC-SD-WM-TCP-007, Rev. 0, Westinghouse Hanford Company, Richland Washington.

Troyer, G. L., 1998, Subject: Chronology of gamma measurement system design and construction (personal communications to R. D. Wilson, Waste Management Federal Services, Inc., Northwest Operations, August 13 and 17), Numatec Hanford Corporation, Richland, Washington.

Troyer, G. L., E. M. Nordquist, D. Legare, K. E. Hillesland, S. F. Kessler, J. V. Nelson, R. F. Richard, S. G. Goodwin, and E. W. Killian, 1998, "High-Performance Gamma-Spectroscopy Measurements of Equipment Retrieved from Hanford Site High-Level Nuclear Waste Tanks," Journal of Radioanalytical and Nuclear Chemistry, Vol. 233, Nos. 1-2, pp. 265-271.

WHC, 1996, Flexible Receiver Radiation Detection System (FRRDS) Users Manual, WHC-SD-W151-UM-001, Rev. 0, Westinghouse Hanford Company, Richland, Washington. 


\section{APPENDIX A1 \\ LLCEDATA AND LLCECALC DATABASE AND LLCEINFO.BIN DESCRIPTION}

This appendix describes the format of the database files generated and maintained by the LongLength Contaminated Equipment (LLCE) waste characterization programs LLCEDATA and LLCECALC. Table A.1-1 shows the database files used by the two programs.

Table A1-1. LLCE Characterization Database Files.

\begin{tabular}{|c|c|c|c|}
\hline Database Name & Used By & Database Format & Description \\
\hline Llceinfo.bin & LLCEDATA & $\begin{array}{l}\text { Encrypted and } \\
\text { compressed* }\end{array}$ & $\begin{array}{l}\text { This database file contains the Long- } \\
\text { Length Contaminated Equipment } \\
\text { (LLCE) physical data, tank waste data, } \\
\text { LLCE geometry factors, waste } \\
\text { container data, radionuclide physical } \\
\text { constants, radionuclide and chemical } \\
\text { regulatory data, and the list of valid } \\
\text { measured gamma isotopes. The } \\
\text { LLCEDATA program user must have } \\
\text { administrative privileges before } \\
\text { permanent changes can be made to } \\
\text { this database. }\end{array}$ \\
\hline Dbchange.mdb & LLCEDATA & MS Access ${ }^{* *} 2.0$ & $\begin{array}{l}\text { This database contains the status } \\
\text { table information that is generated } \\
\text { when editing of the Ilceinfo. bin file is } \\
\text { allowed by LLCEDATA. It maintains a } \\
\text { description of all the changes made to } \\
\text { Iceinfo. bin database by all users of } \\
\text { LLCEDATA. }\end{array}$ \\
\hline Changeblk.fil & LLCEDATA & MS Access 2.0 & $\begin{array}{l}\text { This is a blank dbchange.mdb file. It is } \\
\text { used to create a new dbchange.mab } \\
\text { file when dbchange.mdb becomes too } \\
\text { large. }\end{array}$ \\
\hline $\begin{array}{l}\text { <FILENAME>.EDF } \\
\text { Filename is selected } \\
\text { by program user }\end{array}$ & $\begin{array}{l}\text { LLCEDATA } \\
\text { LLCECALC }\end{array}$ & MS Access 2.0 & $\begin{array}{l}\text { The equipment data file (EDF) is } \\
\text { generated in LLCEDATA and is } \\
\text { required to characterize LLCE waste in } \\
\text { LLCECALC. It contains all data } \\
\text { required to characterize a selected } \\
\text { LLCE. }\end{array}$ \\
\hline Blank.fil & LLCEDATA & MS Access 2.0 & $\begin{array}{l}\text { This is a blank EDF. It is used when a } \\
\text { new EDF is created. }\end{array}$ \\
\hline Report.fil & LLCECALC & MS Access 2.0 & $\begin{array}{l}\text { This is used to create the LLCE } \\
\text { Characterization report in LLCECALC. }\end{array}$ \\
\hline
\end{tabular}

"LLCEINFO.BIN is expanded to create the temporary database file Iceinfo.mdb when the LLCEDATA program starts. L/ceinfo.mdb is an MS Access 2.0-compatible database. Changes to data are made to Iceinfo.mdb and the EDF if the program user has administrative privileges; otherwise, changes are made to the EDF only. When the LLCEDATA program exits, the Iceinfo.mdb database is compacted, encrypted, and stored in the IIceinfo.bin file. Llceinfo.mab is deleted after data have been saved in Ilceinfo.bin.

${ }^{* *}$ MS Access is a trademark of Microsoft Corporation. 


\section{APPENDIX A2 \\ LLCEDATA AND LLCECALC DATABASE FILE STRUCTURE}

The database files used by LLCEDATA and LLCECALC contain one or more database tables. This section will document the database tables that are found in the database files.

\section{A2.1.0 LLCEINFO.BIN DATABASE FILE TABLES}

The Ilceinfo. bin database file is a compressed and encrypted file that is expanded by the LICEDATA program into the database file /lceinfo.mab. L/ceinfo.mdb is the main database file used by LLCEDATA. It contains all of the data necessary to perform waste characterization for any Long-Length Contaminated Equipment (LLCE) retrieved from any underground waste storage tank. The LLCEDATA program is used to update the information in this database.

Table A2-1 shows the database tables that are in /ceinfo. mdb.

Table A2-1. Liceinfo.bin (I/ceinfo.mdb) Database Tables.

\begin{tabular}{|l|l||}
\hline \hline Database Table Name & \multicolumn{1}{|c|}{ Description } \\
\hline Chemreg & Contains a list of all chemical regulatory limits. \\
\hline LLCECont & $\begin{array}{l}\text { Contains physical data for all Long-Length Contaminated Equipment } \\
\text { (LLCE) waste containers. }\end{array}$ \\
\hline LLCEGeom & Contains all known geometry correction factors for LLCE. \\
\hline LLCENuc & Contains physical data for all radionuclides. \\
\hline LLCEPhys & Contains the physical data for all known LLCE. \\
\hline LLCEGamma & Contains the list of all valid measured gamma isotopes. \\
\hline Radreg & Contains the list of all radionuclide regulatory limits. \\
\hline Tankchar & Contains the list of all tank waste characterization data. \\
\hline Element & $\begin{array}{l}\text { Contains the list of all radionuclides and chemicals. Includes description, } \\
\text { chemical abstract services, and chemical formula (for chemicals). }\end{array}$ \\
\hline
\end{tabular}

\section{A2.2.0 DBCHANGE.MDB AND CHANGEBLK.FIL DATABASE TABLES}

The $d b c h a n g e . m d b$ database file contains only one database table. It records all changes made to the Ilceinfo.bin database file during the execution of the LLCEDATA program when the program user is logged in with administrative privileges. Information recorded in the Status database table in $d b c h a n g e . m d b$ is also recorded in the Status database table in the equipment data file (EDF).

The changeblk.fil file is a blank $d b c h a n g e . m d b$ file. When the $d b c h a n g e . m d b$ file size exceeds 5.0 megabytes the LLCEDATA program user is given the option to save the old dbchange.mab file and to create a new empty dbchange.mdb file. Changeb/k. fil is used to create the new dbchange.mdb file. 
Table A2-2 shows the Status database table in the $d b c h a n g e . m d b$ and changeblk.fil database files.

Table A2-2. Dbchange.mdb and Changeb/k.fil Database Tables.

\begin{tabular}{||l|l||}
\hline $\begin{array}{c}\text { Database } \\
\text { Table Name }\end{array}$ & \multicolumn{1}{|c||}{ Description } \\
\hline Status & $\begin{array}{l}\text { This database table contains a detailed description of all changes made to the } \\
\text { Ilceinfo.bin database. This information is recorded during the operation of the } \\
\text { LLCEDATA program when the program user has logged on with administrative } \\
\text { privileges. }\end{array}$ \\
\hline
\end{tabular}

\section{A2.3.0 EDF AND BLANK.FIL DATABASE TABLES}

The EDF is a file generated by the LLCEDATA program. It contains data extracted from the I/ceinfo. bin database file that is applicable to an LLCE selected for retrieval from an underground waste storage tank. In addition, the EDF stores gamma assay data that is generated during the execution of the LLCECALC program. The EDF provides complete documentation for the waste characterization of an LLCE that has been retrieved from an underground waste storage tank. Blank. fil is a blank EDF used to create new EDFs.

Table A2-3 shows the database tables that are in an EDF.

Table A2-3. Equipment Data File (EDF) Database Tables. (2 sheets total)

\begin{tabular}{|l|l|}
\hline $\begin{array}{c}\text { Database } \\
\text { Table Name }\end{array}$ & \multicolumn{1}{c|}{ Description } \\
\hline \hline User & $\begin{array}{l}\text { Contains user data for the EDF. It has general information about the program user, } \\
\text { Long-Length Contaminated Equipment (LLCE) descriptive data, waste tank, units, } \\
\text { and status of what has been completed and edited in the file. }\end{array}$ \\
\hline Chemreg & Contains a list of all chemical regulatory limits for the selected LLCE. \\
\hline LLCECont & $\begin{array}{l}\text { Contains physical data for the waste container that will be used for the selected } \\
\text { LLCE. }\end{array}$ \\
\hline LLCEGeom & Contains geometry correction factors for the selected LLCE. \\
\hline LLCENuc & Contains a copy of the LLCENuc table extracted from the /lceinfo.mdb database file. \\
\hline LLCEPhys & Contains the physical data for the selected LLCE. \\
\hline LLCEGamma & $\begin{array}{l}\text { Contains a copy of the LLCEGamma table extracted from the Ilceinfo.mdb database } \\
\text { file. }\end{array}$ \\
\hline Radreg & Contains a copy of the Radreg table extracted from the Ilceinfo.mab database file. \\
\hline Tankchar & Contains the tank waste characterization data for the selected waste tank. \\
\hline Status & $\begin{array}{l}\text { Contains status of all significant events that takes place in LLCEDATA and } \\
\text { LLCECALC programs. It records all changes and selections made by the program or } \\
\text { program user. }\end{array}$ \\
\hline
\end{tabular}


Table A2-3. Equipment Data File (EDF) Database Tables. (2 sheets total)

\begin{tabular}{|l|l|}
\hline $\begin{array}{c}\text { Database } \\
\text { Table Name }\end{array}$ & \multicolumn{1}{|c|}{ Description } \\
\hline Gammadata & $\begin{array}{l}\text { Initially contains a copy of the Rawdata table data. After gamma assay file } \\
\text { processing, the total LLCE content for each of the constituents. will be found in this } \\
\text { table. }\end{array}$ \\
\hline Rawdata & $\begin{array}{l}\text { Contains the gamma assay information as read from the AV2 gamma assay data } \\
\text { file. }\end{array}$ \\
\hline Positions & $\begin{array}{l}\text { Generated in LLCECALC. Contains }{ }^{137} \text { Cs data extracted from the gamma assay } \\
\text { records including }{ }^{137} \text { Cs inventory from all three detectors, crane position for records, } \\
\text { and the waste tank layer containing the particular part of the LLCE. }\end{array}$ \\
\hline Layer & $\begin{array}{l}\text { Contains LLCECALC estimate of }{ }^{137} \text { Cs content for every tank waste layer defined. } \\
\text { The sum of the }{ }^{137} \text { Cs content for all layers is equal to the total }{ }^{137} \text { Cs content for the } \\
\text { entire LLCE. }\end{array}$ \\
\hline
\end{tabular}

\section{A2.4.0 REPORT.FIL DATABASE TABLES}

The report.fil database file contains only one database table. It is used to create the temporary database file report tmp during execution of the LLCECALC program. This file contains the waste characterization report generated during waste characterization calculations.

Table A2-4 shows the Summary database table in the report fil database file.

Table A2-4. Report.fil Database Tables.

\begin{tabular}{|c|l|}
\hline Database Table Name & \multicolumn{1}{c|}{ Description } \\
\hline \hline Summary & $\begin{array}{l}\text { Contains the LLCE characterization report generated during the } \\
\text { LLCECALC waste characterization process. }\end{array}$ \\
\hline
\end{tabular}




\section{APPENDIX A3 \\ LLCEDATA AND LLCECALC CONFIGURATION FILES}

LLCEDATA and LLCECALC configuration files are ASCII text formatted files and so can be easily read, interpreted, and changed by the user. There is only one configuration file that is used by the LongLength Contaminated Equipment (LLCE) programs.

\section{A3.1.0 LLCECALC.INI CONFIGURATION FILE}

The I/cecalc. ini file contains program initialization shielding correction factors for ${ }^{137} \mathrm{Cs},{ }^{60} \mathrm{Co}$, and ${ }^{154} \mathrm{Eu}$ isotopes and the program initialization parameter for the suspect transuranic (TRU) level. LLCECALC reads the ini file while processing LLCE waste data to determine these values.

Table A3-1 shows the values that are defined in the Ilcecalc.ini file.

Table A3-1. Values Defined in L/cecalc,ini File.

\begin{tabular}{|l|c|l|}
\hline \multicolumn{1}{|c|}{ Heading } & Variable & \multicolumn{1}{c|}{ Description } \\
\hline \multirow{2}{*}{ [137 CS] } & Cor2 & Shielding correction factor for detector No. 2 \\
\cline { 2 - 3 } & Cor3 & Shielding correction factor for detector No. 3 \\
\hline \multirow{2}{*}{ [154EU] } & Cor2 & Shielding correction factor for detector No. 2 \\
\cline { 2 - 3 } & Cor3 & Shielding correction factor for detector No. 3 \\
\hline [60CO] & Cor2 & Shielding correction factor for detector No. 2 \\
\cline { 2 - 3 } & Cor3 & Shielding correction factor for detector No. 3 \\
\hline [SUSPECT] & STRU & $\begin{array}{l}\text { Suspect transuranic (TRU) level (suspect TRU level of } \\
90 \text { nCi/g is entered as 0.90) }\end{array}$ \\
\hline
\end{tabular}

The following text is a printout of the current version of Ilcecalc.ini. This file may be modified by the user to adjust the default values used by LLCECALC. If the comment characters ":" are removed from the shielding correction factor lines, then LLCECALC will use the numerical values supplied and will not auto-calculate the shielding correction factors. The default as supplied /lcecalc. ini file has the shielding factors commented, implying that the default characteristic of LLCECALC will be to auto-calculate the shielding factors.

When the other variables are not commented out (semicolon removed), then LLCECALC reads the value defined in this file, and uses the value in the algorithms. 


\section{Listing of default //ceca/c.In/ file}

$[137 \mathrm{CS}]^{-}$

; The following are calibration (correction factors for detector shielding)

; Correction for detector 1 is always 1.0 so is not specified here.

; Estimate for detector 2 should be good (Commented out to allow autocalc of correction factors)

; variation in detector 3 corrections (Commented out to allow autocalc of correction factors)

;COR2=1.165

;COR3=24.79e6

[154EU]

;COR2 $=1.0$

;COR3 $=1.0$

$[60 \mathrm{CO}]$

;COR2=1.0

;COR3=1.0

[SUSPECT]

; Set the level you want to use to flag LLCE waste as suspect TRU

; Note that entry here is in form of limit ( $\mathrm{nCi} / \mathrm{g}$ ) divided by 100

; i.e. STRU $=0.90$, suspect TRU limit is $90 \mathrm{nCi} / \mathrm{g}$

STRU $=0.85$ 


\section{APPENDIX A4 \\ LLCEDATA AND LLCECALC DATABASE TABLE STRUCTURES}

The database tables in the database files used by LLCEDATA and LLCECALC contain one or more data fields. This section will document the data field structure of each database table. tables.

The following tables explain the terminology used in the description of the fields in the database

Field Type

\begin{tabular}{|l|l|}
\hline Text & Alphanumeric, any letter or number allowed up to field length \\
\hline Integer & Integer number $(-32,767$ to 32,767$)$ \\
\hline Long & $-2,147,483,648$ to $2,147,483,647$ \\
\hline Double & Number entry with 15 significant digits $\left(10^{-307}\right.$ to $\left.10^{+308}\right)$ \\
\hline Byte & Integer entry (0 to 255$)$ \\
\hline Memo & Memo field. Unlimited field size (up to $\sim 1.2$ gbyte text field) \\
\hline Boolean & True or False value \\
\hline
\end{tabular}

Field Size*

\begin{tabular}{|l|l|}
\hline N/A & Size is preset by data type \\
\hline (Number) & Size of field in characters \\
\hline
\end{tabular}

"Only text fields have a size specified

Index Key*

\begin{tabular}{|l|l|}
\hline (Number) & $\begin{array}{l}\text { Designates the fields to be indexed and the sort order for indexing, } \\
\text { starting with } 1 \text { for the first primary field. } \\
\text { Multiple fields in a table can be used as an index key. All index key } \\
\text { fields must have data, and the combination of the field values must be } \\
\text { unique. }\end{array}$ \\
\hline
\end{tabular}

*Not all database tables have Index keys

\section{A4.1.0 CHEMREG TABLE STRUCTURE}

The field structure of the Chemreg database table is shown in Table A4-1. 
Table A4-1. Field Structure of Chemreg Database Table.

\begin{tabular}{|c|c|c|c|c|}
\hline \hline \multicolumn{3}{|c|}{ Table Name: Chemreg } & \multicolumn{3}{|c|}{ Size } & Index Key \\
\hline \hline$\#$ & Field Name & Type & 30 & \\
\hline 1 & Constituent Name $^{\mathrm{a}}$ & Text & 12 & 1 \\
\hline 2 & CAS $^{\mathrm{b}}$ & Text & 4 & \\
\hline 3 & D Code $^{\mathrm{c}}$ & Text & N/A & \\
\hline 4 & D Code Limit $^{d}$ & Double & 12 & \\
\hline 5 & Time $^{\mathrm{e}}$ & Text & 12 & \\
\hline 6 & Date $^{\mathrm{a}}$ & Text & & \\
\hline
\end{tabular}

aConstituent Name is the proper chemical name for the waste constituent.

- CAS is the Chemical Abstracts Service number for the chemical.

'D Code is the Dangerous Waste Number per WAC 173-303-090(8)(C).

'D Code Limit is the $\mathrm{mg} / \mathrm{L}$ limit for a ' $\mathrm{D}$ ' listed chemical. These $\mathrm{D}$-Code limits are found in

WAC 173-303-090(B)(C) as well as in 40 CFR 266, Appendix VII, "Health-Based Limits for Exclusion of Waste-

Derived Residues."

Time and Date are the last time and date when a data record was edited.

40 CFR 266, "Standards for the Management of Specific Hazardous Wastes and Specific Types of Hazardous Waste Management Facilities," Code of Federal Regulations, as amended.

WAC-173-303, "Dangerous Waste Regulations," Washington Administrative Code, as amended.

\section{A4.2.0 ELEMENT TABLE STRUCTURE}

The field structure of the Element database table is shown in Table A4-2.

Table A4-2. Field Structure of Element Database Table.

\begin{tabular}{|c|c|c|c|c|}
\hline \multicolumn{3}{|c|}{ Table Name: Element } & \multicolumn{1}{l|}{} \\
\hline \hline$\#$ & Field Name & Type & Size & Index Key \\
\hline 1 & Description $^{a}$ & Text & 55 & \\
\hline 2 & CAS $^{b}$ & Text & 20 & 1 \\
\hline 3 & Type $^{\mathrm{c}}$ & Text & 15 & \\
\hline 4 & Name $^{d}$ & Text & 55 & \\
\hline 5 & Formula $^{\mathrm{a}}$ & Text & 35 & \\
\hline
\end{tabular}

Description is the description given by the program user for the chemical or radionuclide. In most cases the Description will be the same as Name.

${ }^{\circ} \mathrm{CAS}$ is the Chemical Abstracts Service number of a chemical. For radionuclides the isotope name is recorded as the atomic weight, then the capitalized two-letter abbreviation for the element; e.g., for ${ }^{1{ }^{17} \mathrm{Cs}}$, the CAS would be recorded as $137 \mathrm{CS}$.

'TYpe is 'CHEMICAL', for chemicals and 'RADIOCHEMICAL' for radionuclides.

"Name is the proper name for the chemical or radionuclide.

'Formula is the chemical formula for a chemical. 


\section{A4.3.0 GAMMADATA AND RAWDATA TABLE STRUCTURE}

The database tables Gammadata and Rawdata have an almost identical data structure. The field structure of the these database tables is shown in Table A4-3.

Table A4-3. Field Structure of Gammadata and Rawdata Tables.

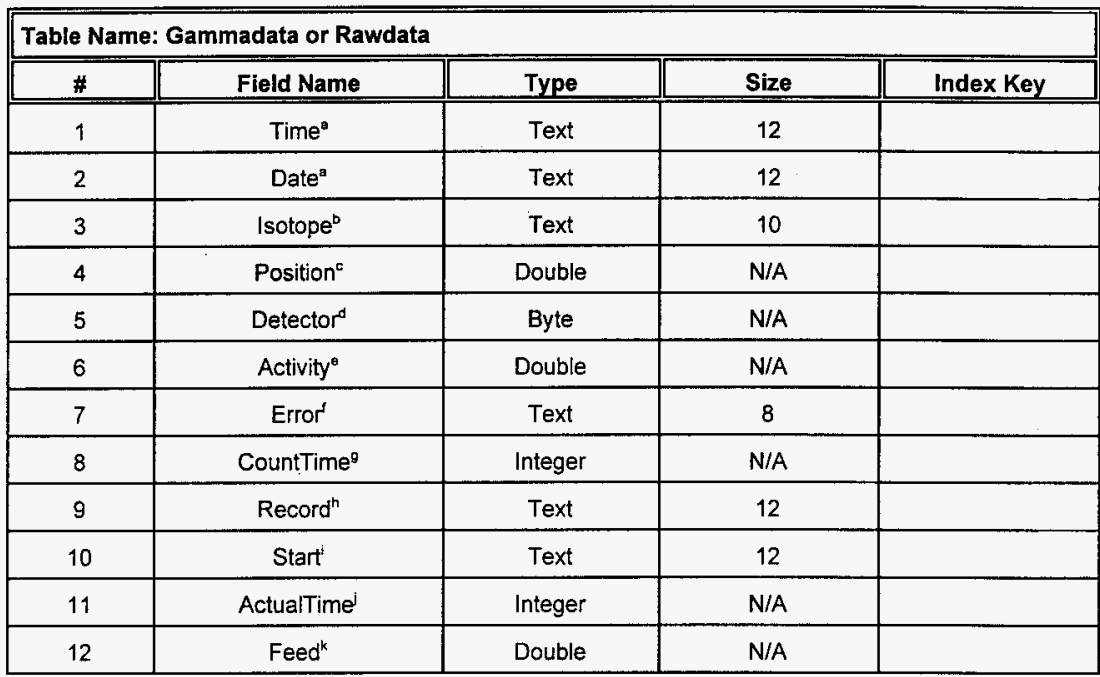

- Time is the time that the reported crane position was recorded. Date is the date the gamma assay record was made.

Isotope is the name of the gamma-emitting radionuclide that was measured. The isotope name is recorded as the atomic weight, then the capitalized two-letter abbreviation for the element; e.g., for ${ }^{137} \mathrm{Cs}$ the Isotope would be recorded as $137 \mathrm{CS}$.

CPosition is the crane position recorded at Time.

Detector is the number of the gamma assay system detector that generated the gamma assay record. This will be number 1,2 , or 3 .

-Activity is the activity in microcuries measured by the Detector.

'Error is the error reported by the gamma assay system for the gamma assay data acquired.

${ }^{\circ}$ Countrime is the time in seconds that the gamma assay counts before producing a new set of gamma acquisition records.

"Record is unique 11-character name given for the spectrum acquired from the Flexible Receiver Radiation Detection System for Rawdata and a 7-digit number (the last 7 characters from Rawdata name) for Gammadata.

'Start time is the start time for data acquisition for the current spectrum from the gamma assay system.

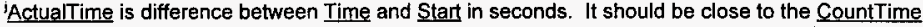
number.

${ }^{k}$ Feed is the crane feed rate (ft/min) for the current data record. This record is currently not used. 


\section{A4.4.0 LAYER TABLE STRUCTURE}

Table A4-4 shows the field structure of the Layer database table.

Table A4-4. Field Structure of Layer Database Table.

\begin{tabular}{|c|c|c|c|c|}
\hline \multicolumn{3}{|c|}{ Table Name: Layer } & & \\
\hline$\#$ & Field Name & Type & Size & Index Key \\
\hline 1 & Isotope $^{\mathrm{a}}$ & Text & 10 & \\
\hline 2 & Number $^{\mathrm{b}}$ & Integer & N/A & \\
\hline 3 & Content $^{\mathrm{c}}$ & Double & N/A & \\
\hline
\end{tabular}

Isotope is the name of the gamma assay system measured radionuclide. Currently the only measured gamma assay isotope is ' $137 \mathrm{CS}$.

"Number is the tank waste layer that this section of the LLCE is in.

c Content is the content in curies of Isotope on the LLCE in Number.

LLCE $=$ Long-Length Contaminated Equipment.

\section{A4.5.0 LLCECONT TABLE STRUCTURE}

The field structure of the LLCECont database table is shown in Table A4-5.

Table A4-5. Field Structure of LLCECont Database Table. (2 sheets total)

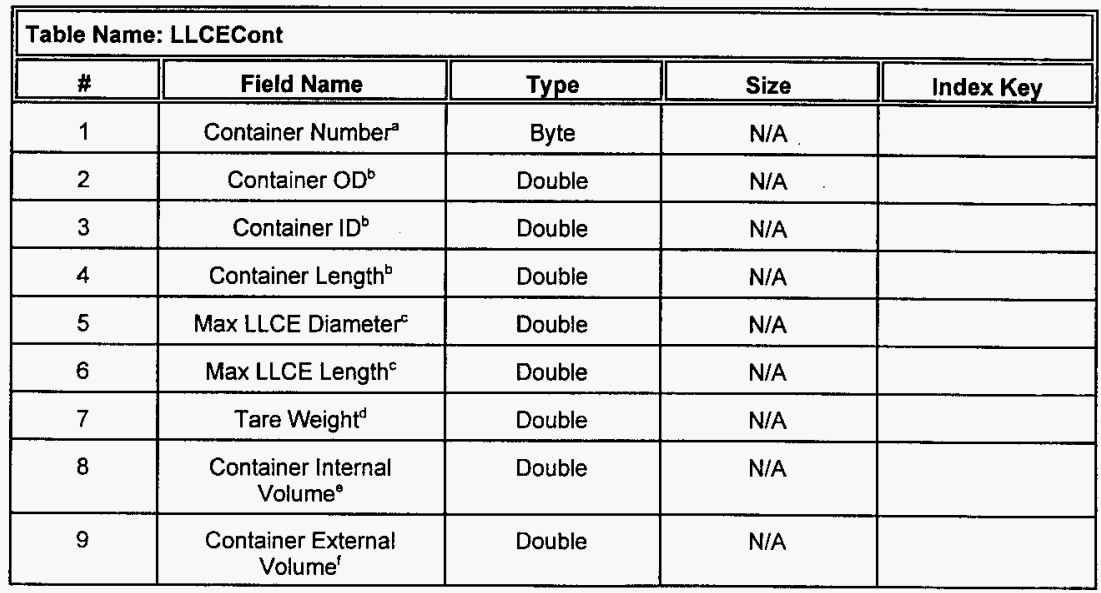


Table A4-5. Field Structure of LLCECont Database Table. (2 sheets total)

\begin{tabular}{|c|c|c|c|c|}
\hline \multicolumn{4}{|c|}{ Table Name: LLCECont } & \multirow[b]{2}{*}{ Index Key } \\
\hline$\#$ & Field Name & Type & Size & \\
\hline 10 & $\begin{array}{c}\text { Container Drawing } \\
\text { Number }\end{array}$ & Text & 30 & \\
\hline 11 & Skid Drawing Number" & Text & 30 & \\
\hline 12 & Skid Weight & Double & N/A & \\
\hline 13 & Time' & Text & 12 & \\
\hline 14 & Datej & Text & 12 & \\
\hline 15 & Descriptionk & Text & 20 & \\
\hline 16 & Max Gross Weight' & Double & N/A & \\
\hline
\end{tabular}
is defined.

Container Number is a sequential number assigned by LLCEDATA when a new LLCE waste container

${ }^{\circ}$ Container $\mathrm{OD}$ is the outside diameter of the waste container in meters. Container ID is the inside diameter of the waste container in meters. Container Length is the overall length of the waste container in meters.

Max LLCE Diameter is the largest-diameter LLCE that will fit in this waste container in meters. Max LLCE Length is the maximum overall length of an LLCE that will fit in this waste container in meters.

Tare Weight is weight of the LLCE waste container in kilograms.

EContainer Internal Volume is the internal volume of the waste container in cubic meters. Default values for the value of Container Internal Volume originates from the SARP(McCormick 1997), and in their absence is calculated by LLCEDATA using:

$$
\text { Internal_Vol }=\left(\frac{\text { Container }_{10}}{2}\right)^{2} \times \pi \times\left(\text { Container }_{\text {Length }}\right)
$$

tContainer External Volume is the external volume of the waste container in cubic meters. Default values for the value of Container External volume originates from the SARP (McCormick 1997), and in their absence is calculated by LLCEOATA using:

$$
\text { External_Vol }=\left(\frac{\text { Container }_{O D}}{2}\right)^{2} \times \pi \times\left(\text { Container }_{\text {Length }}\right)
$$

"Container Drawing Number is the drawing number assigned to the waste container.

hSkid Drawing Number is the drawing number assigned to the skid that goes with this waste container. iSkid Weight is the weight of the skid in kilograms.

Time and Date are the last time and date when this waste container data record was edited.

Description is the name given to this waste container (up to 20 characters). When a new waste container type is defined and no description is entered, LLCEDATA generates a description "LLCE-" + Container Number.

Max Gross Weight is the maximum gross weight allowed for a fully loaded waste container in kilograms.

$I D=$ Inside diameter.

LLCE $=$ Long-Length Contaminated Equipment.

$O D=$ Outside diameter.

SARP $=$ Safety analysis report for packaging.

McCormick, W. A., 1997, Safety Analysis Report for Packaging (Onsite) Long-Length Contaminated Equipment Transport System. HNF-SD-TP-SARP-013, Rev. 0, prepared by Waste Management Federal Services, Inc., Northwest Operations for Fluor Daniel Hanford, Inc., Richland, Washington. 


\section{A4.6.0 LLCEGAMMA}

The field structure of the LLCEGamma database table is shown in Table A4-6.

Table A4-6. Field Structure of LLCEGamma Database Table. (2 sheets total)

\begin{tabular}{|c|c|c|c|c|}
\hline \hline Table Name: LLCEGamma & \multicolumn{3}{|c|}{} \\
\hline \hline$\#$ & Field Name & Type & Size & Index Key \\
\hline 1 & Isotope $^{\mathrm{a}}$ & Text & 6 & 1 \\
\hline 2 & Time $^{\mathrm{b}}$ & Text & 12 & \\
\hline 3 & Date $^{\mathrm{b}}$ & Text & 12 & \\
\hline
\end{tabular}

"Isotope is the name of a radionuclide. The isotope name is recorded as the atomic weight, then the capitalized two-letter abbreviation for the element; e.g., for ${ }^{137} \mathrm{Cs}$ the Isotope would be recorded as $137 \mathrm{CS}$.

'Time and Date are the last time and date that this data record was edited.

\section{A4.7.0 LLCEGEOM TABLE STRUCTURE}

The field structure of the LLCEGeom database table is shown in Table A4-7.

Table A4-7. Field Structure of LLCEGeom Database Table. (2 sheets total)

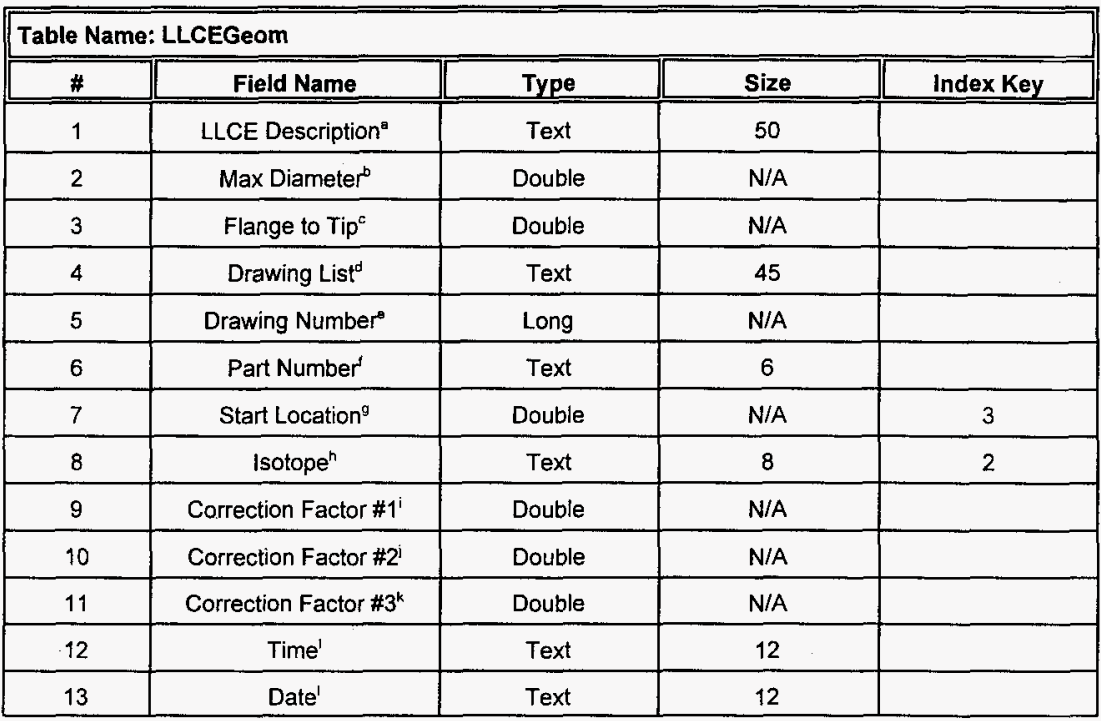


Table A4-7. Field Structure of LLCEGeom Database Table. (2 sheets total)

\begin{tabular}{|c|c|c|c|c|}
\hline \multicolumn{3}{|c|}{ Table Name: LLCEGeom } & & \\
\hline \hline$\#$ & Field Name & Type & Size & Index Key \\
\hline \hline 14 & LLCEID $^{\mathrm{m}, *}$ & Long & N/A & 1 \\
\hline 15 & End Location & Double & N/A & \\
\hline
\end{tabular}

"LLCE Description is the name for the LLCE the geometry factors apply to. This value is identical to the value of the LLCE Description field in the LLCEPhys table.

Max Diameter is the maximum outside diameter in meters for the LLCE the geometry factors apply to. This value is identical to the value in the Max Diameter field in the LLCEPhys table.

'Flange to Tig is the distance in meters from the bottom most tip of the LLCE to the bottom surface of the mounting flange on the LLCE. This value is identical to the value in the Flange to Tip field in the LLCEPhys table.

dDrawing List is the list of drawings that apply to the LLCE. This value is identical to the value of the Drawing List field in the LLCEPhys table.

Drawing Number is the drawing number for the LLCE. This value is identical to the value of the Drawing Number field in the LLCEPhys table.

'Part Number is the part number for the LLCE. This value is identical to the value of the Part Number field in the LLCEPhys table.

"Start Location is the starting location in meters along the length of the LLCE for which the geometry factors in this data record apply. Note that the bottom tip of the LLCE corresponds to a Start Location of zero.

"Isotope is the gamma isotope to which the geometry correction factors will be applied. The Isotope will be one of the valid measured gamma isotopes that are in the LLCEGamma table detector \#1.

'Correction Factor \#1 is a dimensionless multiplication factor that applies to the Isotope for gamma assay detector \#2.

Correction Factor \#2 is a dimensionless multiplication factor that applies to the Isotope for gamma assay assay detector \#3.

${ }^{k}$ Correction Factor \#3 is a dimensionless multiplication factor that applies to the Isotope for gamma

Time and Date are the last time and date when this data record was last edited

"LLCEID is the LLCEDATA-assigned identification number for the LLCE. This value is identical to the value of the 'ID' field in LLCEPhys table.

"End Location is the end location in meters along the length of the LLCE for which the geometry factors in this data record apply. The geometry correction factors apply to the Isotope specified between Start Location and End Location.

is 'ID.'

"Note that field name shown is for equipment data file. For the /lceinfo.mdb database file, the field name

LLCE = Long-Length Contaminated Equipment. 


\section{A4.8.0 LLCENUC TABLE STRUCTURE}

Thë field structure of the LLCENuc database table is shown in Table A4-8.

Table A4-8. Field Structure of LLCENuc Database Table.

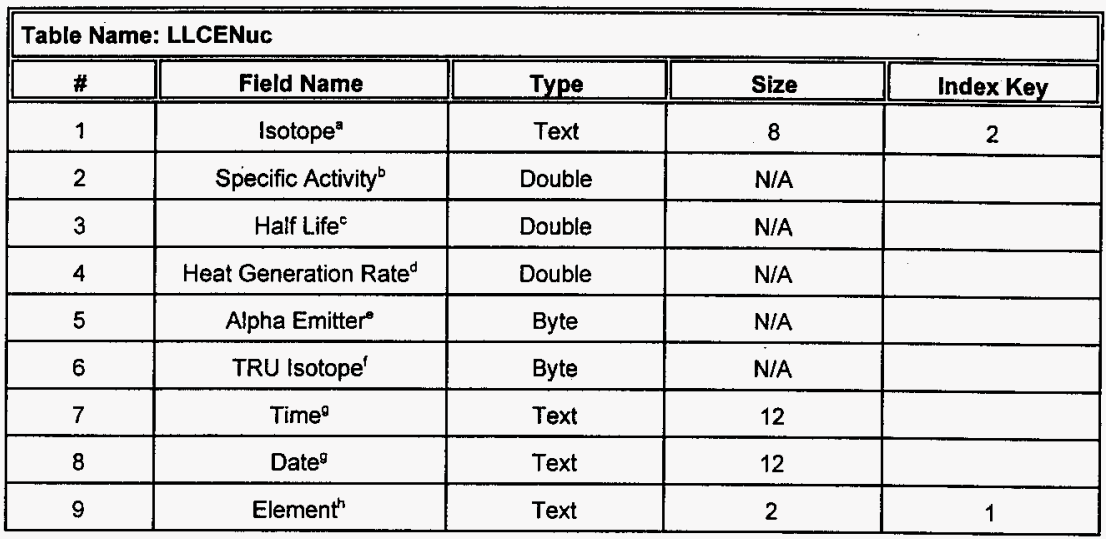

Isotope is the name of a radionuclide. The isotope name is recorded as the atomic weight, then the capitalized one- or two-letter abbreviation for the element; e.g. for ${ }^{137} \mathrm{Cs}$ the Isotope would be recorded as $137 \mathrm{CS}$. 'Specific Activity is the number of curies in one gram of the Isotope $(\mathrm{Ci} / \mathrm{g})$.

cHalf Life is the time it takes for one half of the lsotope to decay in years. (W/Ci).

Heat Generation Rate is the heat generated by radioactive decay of the Isotope in watts per curie

'Alpha Emitter indicates if this Isotope emits an alpha particle when it decays and is a uranium, thorium, or transuranic isotope ( 1 for true and 0 for false).

'TRU Isotope indicates if this Isotope is a transuranic (TRU) radionuclide as specified in WHC-EP-0063-5 (Ellefson 1998) (1 for true and 0 for false).

Time and Date are the time and date this record was last changed.

"Element is the one- or two-letter abbreviation for the Isotope; e.g., for ${ }^{137} \mathrm{Cs}$, Element = 'CS.'

Ellefson, M. D., 1998, Hanford Site Solid Waste Acceptance Criteria, HNF-EP-0063-5, prepared by Waste Management Federal Services of Hanford, Inc., for Fluor Daniel Hanford, Inc., Richland, Washington. 


\section{A4.9.0 LLCEPHYS TABLE STRUCTURE}

The field structure of the LLCEPhys database table is shown in Table A4-9.

Table A4-9. Field Structure of LLCEPhys Database Table.

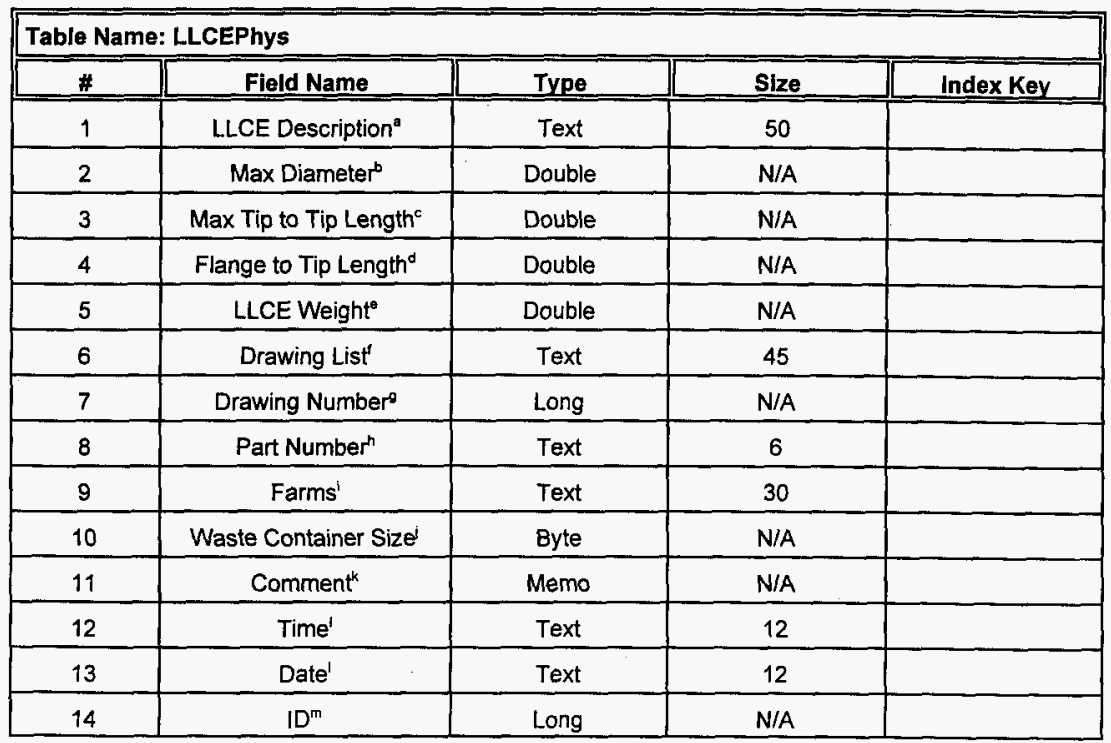

LLCEE Description is the name for the LLCE; i.e., Profile Thermocouple.

Max Diameter is the maximum outside diameter in meters for the LLCE. This dimension represents the smallest-jnside-diameter pipe that the LLCE could be fit through.

Max Tip to Tip Length is the tip-to-tip length of the LLCE in meters. This dimension represents the smallest length of pipe that the LLCE would fit in.

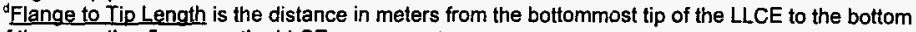
surface of the mounting flange on the LLCE.

"LLCEE Weight is the dry, clean weight of the LLCE in kilograms.

'Drawing List is the list of drawings that are applicable to the LLCE. This list comes from the main assembly drawing for the LLCE.

Drawing Number is the numerical suffix part of the drawing number for the LLCE; e.g., for drawing H-2-32014 the drawing number would be '32014.' This should be the main assembly drawing for the LLCE.

"Part Number is the part number as specified on the LLCE drawing for the LLCE.

Farms is the tank farms for this LLCE that is listed on the LLCE drawing(s).

Waste Container Size this is the container number (from LLCECont table) that is the smallest container size into which the LLCE will fit.

Comment is any comment the program user wishes to make about an LLCE. For instance the program user may wish to record the riser and tanks from which this LLCE has been retrieved.

Time and Date are the last time and date that this data record was edited. record is added.

ID is the unique identification number that is automatically generated by LLCEDATA when a new data

$$
\text { LLCE = Long-Length Contaminated Equipment. }
$$




\section{A4.10.0 POSITIONS TABLE STRUCTURE}

The field structure of the Positions database table is shown in Table A4-10.

Table A4-10. Field Structure of Positions Database Table.

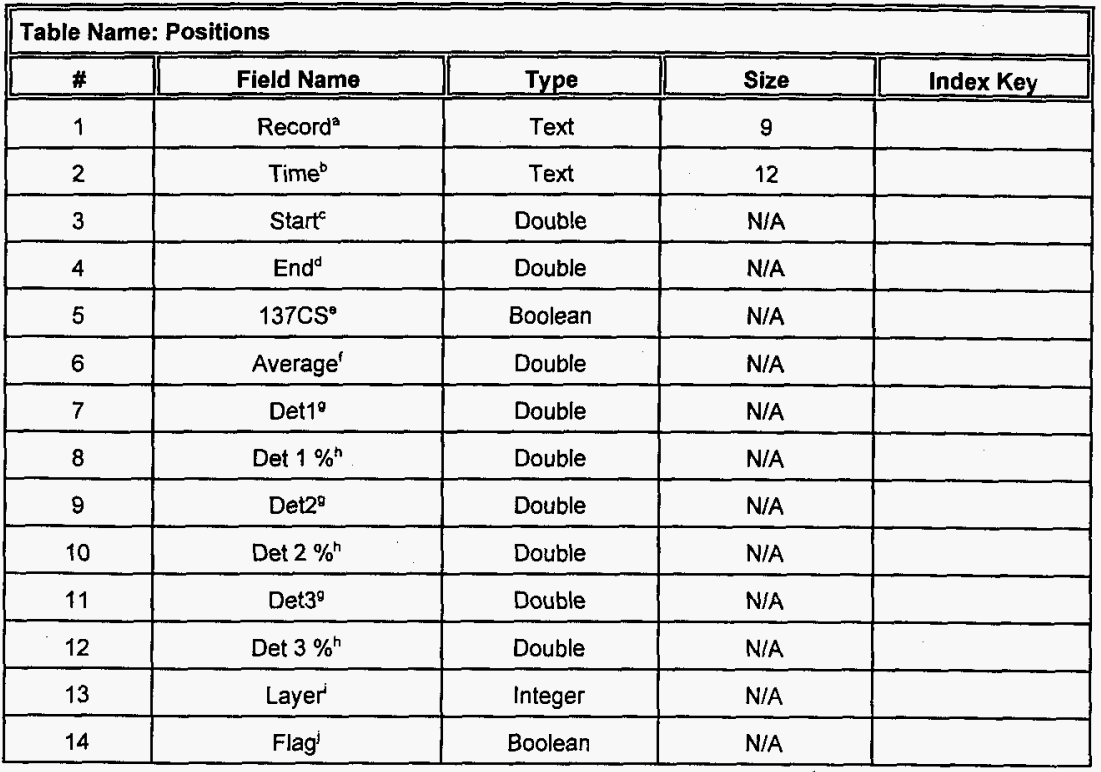

system.

"Record is the last seven characters of the spectrum record ID number generated by the gamma assay

'Time is the time the gamma assay spectrum was made.

'Start is the start position in meters for spectrum ID Record.

DEnd is the end position in meters for spectrum ID Record.

FALSE)

- $137 \mathrm{CS}$ is a flag that indicates if ${ }^{137} \mathrm{Cs}$ activity is recorded for spectrum ID Record (-1 for TRUE and 0 for

'Average is the weighted average ${ }^{137} \mathrm{Cs}$ activity in Ci/ft for this record.

Det1, Det2, and Det3 are the recorded ${ }^{137} \mathrm{Cs}$ activity (Ciffi) for this spectrum ID Record.

Det $1 \%$, Det $2 \%$, and Det $3 \%$ are errors reported by gamma assay system for detectors 1,2 , and 3 for the ${ }^{137} \mathrm{Cs}$ measurements in this record.

Layer is the tank waste layer number in which this spectrum ID Record is found.

Flag is currently an extra unused field.

\section{A4.11.0 RADREG TABLE STRUCTURE}

The field structure of the Radreg database table is shown in Table A4-11. 
Table A4-11. Field Structure of Radreg Database Table.

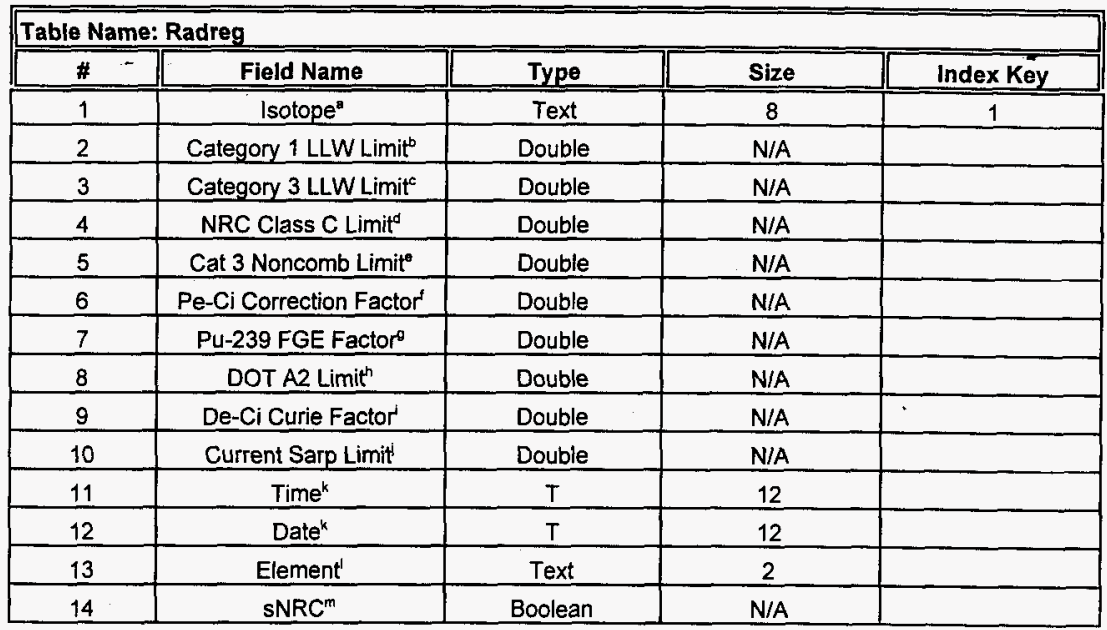

Isotope is the name of a radionuclide. The isotope name is recorded as the atomic weight, then the capitalized one- or two-letter abbreviation for the element; e.g., for ${ }^{137} \mathrm{Cs}$ the Isotope would be recorded as $137 \mathrm{CS}$.

${ }^{b}$ Category 1 LLW Limit is the limit for a waste package for the Isotope in curies per cubic meter (Ci/m $\left.\mathrm{m}^{3}\right)$ for Category 1 Low-Level Waste (LLW) Disposal. The limits will be as specified in HNF-EP-0063-5 (Ellefson 1998).

"Category 3 LLW Limit is the limit for a waste package for the Isotepe in curies per cubic meter $\left(\mathrm{Ci} / \mathrm{m}^{3}\right)$ for Category 3 Low-Level Waste Disposal. The limits will be as specified in Ellefson (1998).

NRC Class C Limit is the limit for a waste package for the Isotope for NRC class C. The limits will be as specified in (Eliefson 1998). Units are $\mathrm{Ci} / \mathrm{m}^{3}$ except for ${ }^{241} \mathrm{Pu}$ and ${ }^{242} \mathrm{Cm}$ where the units are in $\mathrm{nCi} / \mathrm{g}$.

- Cat 3 Noncomb Limit is the limit for a waste package for the Isotope in curies per cubic meter $\left(\mathrm{Ci} / \mathrm{m}^{3}\right)$ for Category 3 Level Waste Disposal of a noncombustible waste package. The limits will be as specified in Ellefson (1998).

'PE-Ci Correction Factor is the multiplication factor applied to the Isotope to calculate the equivalent ${ }^{239} \mathrm{Pu}$ content of a waste package. The limits will be as specified in Ellefson (1998).

${ }^{9} \mathrm{Pu}-239$ FGE Factor is the multiplication factor applied to the Isotope to calculate the equivalent ${ }^{239} \mathrm{Pu}$ fissile gram equivalent content of a waste package. The limits will be as specified in Ellefson (1998).

'DOT A2 Limit is the limit for a waste package for the Isotope in curies for a Type A waste package. The limits will be as specified in the current 49 CFR 173.

DE-Ci Curie Factor is the multiplication factor applied to the Isotope to calculate the dose equivalent curies (DE-Ci) in a waste package. The limits will be as specified in Ellefson (1998).

'Current SARP Limit is the curie limit for the Isotope as established in the SARP (McCormick 1997) for the waste package.

kTime and Date is the time and date this record was last changed.

'Element is the one- or two-letter abbreviation for the Isotope; e.g., for ${ }^{137} \mathrm{Cs}$, Element = 'CS.'

"sNRC is TRUE (-1) when units for NRC Class C Limit for the Isotope is in nCi/gram else this field is FALSE (0).

DOT = U.S. Department of Transportation.

NRC = U.S. Nuclear Regulatory Commission.

SARP $=$ Safety analysis report for packaging.

49 CFR 173, "Shippers--General Requirements for Shipments and Packagings," Code of Federal Regulations, as amended.

Ellefson, M. D., 1998, Hanford Site Solid Waste Acceptance Criteria, HNF-EP-0063-5, prepared by Waste Management Federal Services of Hanford, Inc., for Fluor Daniel Hanford, Inc., Richland, Washington. McCormick, W. A., 1997, Safety Analysis Report for Packaging (Onsite) Long-Length Contaminated Equipment Transport System, HNF-SD-TP-SARP-013, Rev. 0, prepared by Waste Management Federal Services, Inc., Northwest Operations for Fluor Daniel Hanford, Inc., Richland, Washington. 


\section{A4.12.0 STATUS TABLE STRUCTURE}

The field structure of the Status database table is shown in Table A4-12.

Table A4-12. Field Structure of Status Database Table.

\begin{tabular}{|c|c|c|c|c|}
\hline \multicolumn{2}{|c|}{ Table Name: Status } & Type & Size & Index Key \\
\hline \hline$\#$ & Field Name & Nemo & N/A & \\
\hline 1 & Line $^{\mathrm{a}}$ & Long & N/A & \\
\hline 2 & ID $^{\mathrm{b}}$ & Lemo & \\
\hline
\end{tabular}

"Line contains one status line from the LLCEDATA or LLCECALC program. Each line is prefixed with the date and time the line was generated.

$\underline{\underline{D}}$ is a auto-incrementing field that automatically generates a new ID number for each Line saved.

$\mathrm{ID}=$ Identification.

\section{A4.13.0 SUMMARY TABLE STRUCTURE}

The field structure of the Summary database table is shown in the Table A4-13.

Table A4-13. Field Structure of Summary Database Table.

\begin{tabular}{|c|c|c|c|c|}
\hline \hline \multicolumn{2}{|c|}{ Table Name: Summary } \\
\hline \hline$\#$ & Field Name & Type & Size & index Key \\
\hline 1 & Line & Memo & N/A & \\
\hline
\end{tabular}

${ }^{*}$ Contains the line by line waste characterization report generated by LLCECALC.

\section{A4.14.0 TANKCHAR TABLE STRUCTURE}

The field structure of the Tankchar database table is shown in Table A4-14. 
Table A4-14. Field Structure of Tankchar Database Table.

\begin{tabular}{|c|c|c|c|c|}
\hline$\#$ & Field Name & Type & Size & Index Key \\
\hline 1 & Tank $^{a}$ & Text & 16 & $1^{*}$ \\
\hline 2 & Constituent & Text & 35 & 3 \\
\hline 3 & $\mathrm{CAS}^{\mathrm{C}}$ & Text & 14 & \\
\hline 4 & Unit Mass $^{\alpha}$ & Text & 3 & \\
\hline 5 & Unit Volume & Text & 4 & \\
\hline 6 & Layer Content & Double & N/A & \\
\hline 7 & Layer Number & Byte & $N / A$ & 4 \\
\hline 8 & Layer Thickness ${ }^{h}$ & Double & N/A & \\
\hline 9 & SpGi & Double & N/A & \\
\hline 10 & Data Source' & Text & 128 & \\
\hline 11 & Time $^{k}$ & Text & 12 & \\
\hline 12 & Date $^{*}$ & Text & 12 & \\
\hline 13 & Type' & Text & 14 & 2 \\
\hline 14 & Content $^{m}$ & Double & N/A & \\
\hline
\end{tabular}

*Index keys are, in order, Tank, Type, and CAS data fields in the /lceinfo.bin database table Tankchar.

"Tank is name of the tank. It is in the form of facility number + tank farm + tank number; e.g., Tank 102 in AP tank farm would be 241-AP-102.

bonstituent is the name of the chemical or radionuclide that is a constituent in the Tank waste.

'CAS for radionuclides is defined as the atomic weight of the isotope followed by the one- or two-letter abbreviation for the element; e.g., for ${ }^{137} \mathrm{Cs}$ the CAS would be $137 \mathrm{CS}$. For chemicals CAS is the Chemical Abstracts Service name for the chernical.

"Unit Mass is grams for chemicals or curies for radionuclides. The LLCEDATA program converts all Unit Mass entries into $\mathrm{g}$ and $\mathrm{Ci}$.

"Unit Volume is the volume unit defined for the tank waste. Volume.

'Laver Content is the content of the Constituent in the tank waste in the defined Unit Mass and Unit

LLayer Number is the tank waste layer containing the Constituent. Multiple tank waste layers may be defined for a waste tank where each layer has a different waste composition. Tank Layer Numbers start at the bottommost layer defined for a waste tank and proceed sequentially through the tank waste layers to the topmost defined layer in the tank.

hLayer Thickness is the thickness in meters for the current Layer Number. For a single layer tank waste model, the Layer Thickness will be zero.

'SpG is the specific gravity for the Laver Number.

'Data Source is a text field that allows a description to be added for the source of tank waste data.

KTime and Date are the last time and date this record was edited.

'Type is 'CHEMICAL' for chemicals and 'RADIOCHEMICAL' for radionuclides.

${ }^{m}$ Content is the actual Long-Length Contaminated Equipment content of the Constituent, grams for chemicals and $\mathrm{Ci}$ for radionuclides, in the current Layer Number. This field is calculated in the LLCECALC program. 


\section{A4.15.0 USER TABLE STRUCTURE}

The field structure of the User database table is shown in Table A4-15.

Table A4-15. Field Structure of User Database Table. (2 sheets total)

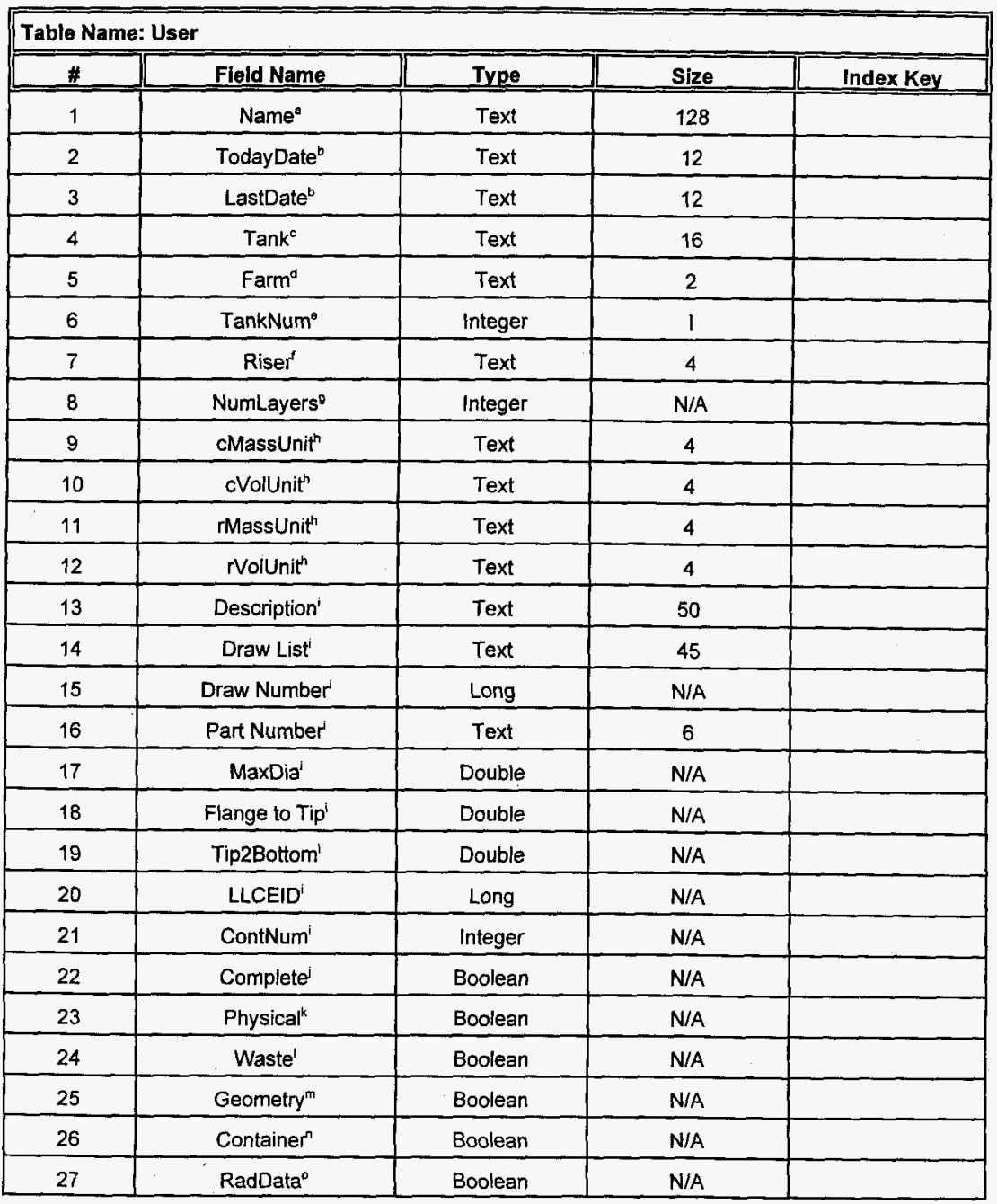


Table A4-15. Field Structure of User Database Table. (2 sheets total)

\begin{tabular}{|c|c|c|c|c|}
\hline \multicolumn{4}{|c|}{ Table Name: User } & \multirow[b]{2}{*}{ Index Key } \\
\hline$\#$ & Field Name & Type & Size & \\
\hline 28 & RadReg' & Boolean & N/A & \\
\hline 29 & ChemReg ${ }^{q}$ & Boolean & $\mathrm{N} / \mathrm{A}$ & \\
\hline 30 & Gamma' & Boolean & N/A & \\
\hline 31 & Gammalnput ${ }^{5}$ & Boolean & N/A & \\
\hline 32 & GammaFile' & Text & 128 & \\
\hline
\end{tabular}

"Name is the user name entered by the LLCEDATA program user.

"TodayDate is today's date. LastDate is the last date the equipment data file (EDF) was edited.

Tank is identical to the Tank field in the Tankchar data table.

'Farm is the tank farm identity from the Tank field; e.g., AX, SY, AZ. A.

TankNum is the tank number from the Tank field; e.g., for 241-AZ-101, TankNum would be equal to

'101.'

'Riser is the riser number (if any) entered by LLCEDATA program user when selecting waste tank for

Long-Length Contaminated Equipment (LLCE) retrieval.

- NumLayers is the number of different tank waste layers defined in the Tankchar data table for Tank.

ccMassunif and cVolunit are the mass unit and volume unit selected for chemicals defined in the

Tankchar data table. Massunit and $\mathrm{r}$ olunit are the mass unit and volume unit selected for radionuclides defined in the Tankchar data table. Note that cVolUnit and rVolUnit should be identical.

'Description, Draw List, Draw Number, Part Number, MaxDia, Flange to Tip, Tip2Bottom. LLCEID, and ContNum are respectively the same as data fields 'LLCE Description,' 'Drawing List,' 'Drawing Number,.' 'Part Number,' 'Max Diameter, 'Flange to Tip Length,' 'Max Tip to Tip Length,' 'ID,' and 'Waste Container Size' in the LLCEPhys data table. program.

'Complete is set to TRUE when ail required input to the EDF has been completed in the LLCEDATA

"Physical is set to TRUE when an LLCE has been selected in LLCEDATA and LLCEPhys data table data has been saved to the EDF.

'Waste is set to TRUE when a waste tank has been selected and tank waste characterization data have been saved to the EDF.

"Geometry is set to TRUE when geometry correction factors have been defined for the selected LLCE and have been saved to the EDF.

"Container is set to TRUE when the LLCE waste container data is edited.

RadData is set to TRUE when the radionuclide constant data is edited.

RadReg is set to TRUE when the radionuclide regulatory limit data is edited.

"ChemReg is set to TRUE when the chemical regulatory limit data is edited.

'Gamma is set to TRUE when the list of valid measured gamma isotopes has been edited. saved in the EDF.

Gammalnput is set to TRUE in the LLCECALC program after gamma assay data has been read and

'GammaFile is set to the full path of the gamma assay input file (AV2 file) once gamma assay data is read and saved in the EDF file. 


\section{A4.16.0 REFERENCES}

40 CFR 266, "Standard for the Management of Specific Hazardous Wastes and Specific Types of Hazardous Waste Management Facilities," Code of Federal Regulations, as amended.

49 CFR 173, "Shippers--General Requirements for Shipments and Packagings," Code of Federal Regulations, as amended.

WAC-173-303, "Dangerous Waste Regulations, Washington Administrative Code, as amended.

Ellefson, M. D., 1998, Hanford Site Solid Waste Acceptance Criteria, HNF-EP-0063-5, prepared by Waste Management Federal Services of Hanford, Inc., for Fluor Daniel Hanford, Inc., Richland, Washington.

McCormick, W. A., 1997, Safety Analysis Report for Packaging (Onsite) Long-Length Contaminated Equipment Transport System, HNF-SD-TP-SARP-013, Rev. 0, prepared by Waste Management Federal Systems, Inc., Northwest Operations for Fluor Daniel Hanford, Inc., Richland, Washington. 


\section{APPENDIX B \\ DESCRIPTION OF THE AV2 FILE FORMAT}

\section{B.1.0 SAMPLE AV2 FILE}

When the program LLCECALC starts, the user is asked to select two files, the equipment data file (EDF) and the gamma assay (AV2) file. The EDF is created by LLCEDATA. The methodology for creating the EDF is presented in Volume I, the user's manual, and a technical discussion of its use and contents is presented in Section 2.3 of this volume. The AV2 file is created by the Gamma Energy Analysis System, a component of the Flexible Receiver Radiation Detection System. This section will describe the format and content of the AV2 file and discuss how LLCECALC uses the information contained in the AV2 file.

To serve as a means to explain the contents of an AV2 file, Table B-1 shows the actual content of a real AV2 file. The file used is the $1101 a z 13 d$.av2 file that is part of verification case three in the user's manual.

Table B-1. Sample (First Few Lines) AV2 File: t101az13d.av2

\begin{tabular}{|c|c|c|c|c|c|c|c|c|c|c|}
\hline Spectrum & Date & Time & Time & Date & Time & Position & Nuclìd & & Activity & Error \\
\hline \multirow[t]{2}{*}{ A.1650807360 } & 50896 & $07: 36: 17$ & 60. & 8-MAY-1996 & $7: 37: 17$ & -833 & & & & \\
\hline & & & & & & & $55 \mathrm{Cs}$ & 137 & $-3.798 \mathrm{E}-02$ & 77.38 \\
\hline \multirow[t]{2}{*}{ A1 650807370} & 50896 & $07: 37: 24$ & 60. & 8-MAY-1996 & $7: 38: 26$ & -1954 & & & & \\
\hline & & & & & & & $55 \mathrm{CS}$ & 137 & $-1.149 \mathrm{E}-01$ & $22.6 \frac{2}{8}$ \\
\hline \multirow[t]{3}{*}{ A1650807380 } & 50896 & $07: 38: 32$ & 60. & B-MAY-1996 & $7: 39: 32$ & -3031 & & & & \\
\hline & & & & & & & $27 \mathrm{Co}$ & 57 & $2.316 \mathrm{E}-02$ & 72.28 \\
\hline & & & & & & & $55 \mathrm{Cs}$ & 137 & $-5.836 \mathrm{E}-02$ & 73.58 \\
\hline \multirow[t]{2}{*}{ A1650803390 } & 50896 & $07: 39: 34$ & 60 & 8-MAY-1996 & $7: 40: 35$ & -4057 & & & & \\
\hline & & & & & & & $55 \mathrm{cs}$ & 237 & $-7.597 \mathrm{E}-02$ & 45.58 \\
\hline \multirow[t]{2}{*}{ Al 650807400} & 50896 & $07: 40: 37$ & 60. & $8-M A Y-1996$ & $7: 41: 38$ & -5079 & & & & \\
\hline & & & & & & & $55 \mathrm{cs}$ & 137 & $-1.165 \mathrm{E}-01$ & $19.7 \mathrm{z}$ \\
\hline \multirow[t]{4}{*}{ A 1650807420} & 50896 & $07: 42: 00$ & 60 & 8-MAY-1996 & $7: 43: 02$ & -6087 & & & & \\
\hline & & & & & & & $11 \mathrm{NA}$ & 22 & $-2.495 \mathrm{E}-03$ & 546.78 \\
\hline & & & & & & & $550 s$ & 137 & $-1.077 E-01$ & 26.58 \\
\hline & & & & & & & $63 \mathrm{EU}$ & 154 & $-7.025 \mathrm{E}-03$ & 546.78 \\
\hline
\end{tabular}

The column headings used in the listing above come from the AV2 file. The first column, Spectrum, is used by LLCECALC to identify the detector number $(1,2$, or 3$)$ and provides the unique record ID number. This is discussed in Section 2.3.1, step 4.

The column Position is the crane position at the end of a gamma spectrum reading. These measurement can be in feet or in thousandths of a foot. Section 2.3.2.1 discusses the methodology of how this determination is made. It is possible for these position measurements to be zero, and Section 2.3.2.2 discuses this case. 
The column Nuclide contains the isotope that is measured in the Activity column. Currently, the only isotopes LLCECALC uses is ${ }^{137} \mathrm{Cs}$. The other isotopes are ignored. The Activity column reports the GEA measured activity for the isotope in units of micro-curries. The Error column is a measure of the percent ertor for the activity recorded for the specific isotope. Although the example listing above only show a few lines in the AV2 file, the AV2 file contains isotope readings (and percent errors) for up to three detectors for any one record. The percent error values are used to compute a statistically weighted average from the three detector readings.

\section{B.2.0 STATISTICALLY WEIGHTED AVERAGE FOR AV2 ACTIVITY MEASUREMENTS}

When different detectors independently measure the same physical quantity, such as LLCE gamma activity at a particular position, then the proper way to average the result of these duplicate measurements is to weigh each contributor to the average by a factor inversely related to its statistical precision. That is, for small values of precision (or of statistical error) the statistical weight should be high. Statistically, the variance is a measure of confidence for a measurement and is defined as the square of the precision or statistical error value. The statistical weight therefore is taken as the reciprocal square of the statistical error.

The statistically weighted average is computed by multiplying each measured activity by the reciprocal square of the error, summing this quantity for all detectors, and dividing by the sum of the reciprocal squared errors (Bevington 1969). An assumption implicit in this method is that each measured gamma activity is independent of the other and each detector samples the same gamma source. Neither of these assumptions is strictly true for two reasons: (1) the efficiency of detectors No. 2 and No. 3 are dependent on detector No. 1 because of the shielding correction factors applied by LLCECALC (see Section 1.4.2) and (2) the detectors are arrayed around the circumference of the LLCE, separated by 120 degrees, so that they view different azimuthal portions of the contamination source. The statistical fluctuations for each detector reading, however, are still independent of one another. Even though the detectors do not sample exactly the same gamma source, it is still a better procedure to compute the statistically weighted average rather than a simple average because the detectors have such widely differing gamma detection efficiencies and hence exhibit large differences in percent error.

For the three detectors of the Flexible Receiver Radiation Detection System, the gamma spectra analysis software (Killian and Hartwell 1988, Killian and Femec 1992, Killian and Sorensen 1992) extracts the net counts/s in the ${ }^{137} \mathrm{Cs}\left({ }^{137 m} \mathrm{Ba}\right)$ gamma peak at $662 \mathrm{keV}$ and reports an error that is related to the statistics of the detection and peak shape analysis process. This percent error is a measure of statistical precision and therefore can be used as the basis for computing the statistical weighting factor.

The AV2 file displayed in Section B.1.0 above, from the verification directory, has percent error values as high as $77.3 \%$ for ${ }^{137} \mathrm{Cs}$. In this particular case, this largest error is the first reading of ${ }^{137} \mathrm{Cs}$ in the AV2 file, implying an anomaly could have caused the large error. The status report identifies this first record as being discarded due to its location above the mounting flange of the LLCE. This occurs for the second largest error also, just below the first record. Typical percent activity errors for ${ }^{137} \mathrm{Cs}$ range from $1 \%$ to $20 \%$, with a few as high as $35 \%$, for this particular file.

Using the stated definition of the statistically weighted average, for the three detectors of the LLCE gamma measurement system, the weighted average is 


$$
\text { Average }=\frac{\sum_{i=1}^{3} \text { Act }_{1} \times\left(1 / \text { Err }_{1}\right)^{2}}{\sum_{i=1}^{3}\left(1 / \text { Err }_{1}\right)^{2}}
$$

where, Act, is the activity measured by detector \# $\mathbf{i}$

Err, is the percent error for detector \# $I$ activity.

Average is the statistically weighted average activity.

The effect of statistical weighting on the averaging process is to weigh most heavily those detector activities with the smallest percent error. For example, if detector No. 1 measures 1,000 $\mu \mathrm{Ci} / \mathrm{ft}$, detector No. 2 measures $1,500 \mu \mathrm{Ci} / \mathrm{ft}$, and detector No. 3 measures $2,000 \mu \mathrm{Ci} / \mathrm{ft}$, then the simple average activity is $1,500 \mu \mathrm{Ci} / \mathrm{ft}$. This would be the correct average activity if all percent error values were the same. However, if detector No. 1 has $1 \%$ error while detectors No. 2 and No. 3 have $10 \%$ error, the statistical weighted average is $1,015 \mu \mathrm{Ci} / \mathrm{ft}$, very close to the value of $1,000 \mu \mathrm{Ci} / \mathrm{ft}$ for detector No. 1 alone. If detector No. 3 has $1 \%$ error while detectors No. 1 and No. 2 have $10 \%$ error, the statistical weighted average is $1,985 \mu \mathrm{Ci} / \mathrm{tt}$, very close to the value of $2,000 \mu \mathrm{Ci} / \mathrm{ft}$ for detector No. 3 alone. This illustrates the concept of statistical weighting of the detector activities.

Section 3.1.3 contains an example calculation for one of the verification test cases validating LLCECALCs methodology in performing this statistically weighted average calculation.

\section{B.3.0 REFERENCES}

Bevington, P. R., 1969, Data Reduction and Error Analysis for the Physical Sciences, McGraw-Hill Book Co., New York, New York.

Killian, E. W., and D. A. Femec, 1992, An Operator's Guide for VAXGAP: A Gamma-ray Spectral Analysis Package, EGG-2672, Edgerton, Germeshausen \& Grier, Idaho Falls, Idaho.

Killian, E. W., and T. C. Sorensen, 1992, "Concurrent Determination of Ambient Background Contribution to Low-Activity High-Resolution Gamma-Ray Spectra," Radioactivity \& Radiochemistry, Vol. 3 No. 1.

Killian, E. W., and J. K. Hartwell, 1988, VAXGAP: A Code for the Routine Analysis of Gamma-Ray Pulse-Height Spectra on a VAX Computer, EGG-2633, Edgerton, Germeshausen \& Grier, Idaho Falls, Idaho. 
DISTRIBUTION SHEET

\begin{tabular}{|c|c|c|c|c|c|}
\hline \multirow{2}{*}{$\begin{array}{l}\text { To } \\
\text { Distribution }\end{array}$} & \multirow{2}{*}{\multicolumn{3}{|c|}{$\begin{array}{l}\text { From } \\
\text { Packaging Engineering } \\
\end{array}$}} & \multicolumn{2}{|l|}{ Page 1 of 1} \\
\hline & & & & \multicolumn{2}{|c|}{ Date August 27,1998} \\
\hline \multicolumn{4}{|c|}{ Project Title/Work Order } & \multicolumn{2}{|c|}{ EDT No. 622941} \\
\hline \multicolumn{4}{|c|}{$\begin{array}{l}\text { LLCEDATA and LLCECALC for Windows Version 1.0, Volume II: } \\
\text { Technical Manual (HNF-3169, Rev. 0) }\end{array}$} & \multicolumn{2}{|c|}{ ECN No. N/A } \\
\hline Name & MSIN & $\begin{array}{c}\text { Text } \\
\text { With All } \\
\text { Attach. }\end{array}$ & Text Only & $\begin{array}{l}\text { Attach./ } \\
\text { Appendix } \\
\text { Only } \\
\end{array}$ & $\begin{array}{l}\text { EDT/ECN } \\
\text { Only }\end{array}$ \\
\hline $\begin{array}{l}\text { R. R. Connolly } \\
\text { D. W. Crass (2) } \\
\text { M. D. Ellefson } \\
\text { J. G. Field } \\
\text { D. C. Hetzer } \\
\text { D. E. Legare } \\
\text { W. A. McCormick } \\
\text { J. C. McCoy } \\
\text { J. G. McFadden (2) } \\
\text { M. A. McGhan } \\
\text { D. L. Riley } \\
\text { H. L. Roach } \\
\text { T. Romano } \\
\text { R. D. Wilson } \\
\text { Document Processing Center } \\
\text { HNF-3169 File } \\
\text { Work Control (D. Kelly) } \\
\end{array}$ & $\begin{array}{l}\text { T4-05 } \\
\text { R3-47 } \\
\text { T3-05 } \\
H 1-15 \\
\text { S6-31 } \\
\text { R3-47 } \\
H 1-15 \\
H 1-15 \\
H 1-15 \\
H 1-15 \\
H 1-15 \\
H 5-57 \\
H 1-15 \\
H 1-15 \\
\text { A3-94 } \\
H 1-15 \\
H 1-15 \\
\end{array}$ & $\begin{array}{l}x \\
x \\
x \\
x \\
x \\
x \\
x \\
x \\
x \\
x \\
x \\
x \\
x \\
x \\
x \\
x\end{array}$ & & & $X$ \\
\hline Centrac Files & $8 /-07$ & $x$ & & & \\
\hline
\end{tabular}

